\title{
Deformation of Codimension-2 Surface and Horizon Thermodynamics
}

\author{
Li-Ming Cao* \\ Department of Physics, Kinki University, \\ Higashi-Osaka, Osaka 577-8502, Japan,
}

October 18, 2018

\begin{abstract}
The deformation equation of a spacelike submanifold with an arbitrary codimension is given by a general construction without using local frames. In the case of codimension- 1 , this equation reduces to the evolution equation of the extrinsic curvature of a spacelike hypersurface. In the more interesting case of codimension-2, after selecting a local null frame, this deformation equation reduces to the well known (cross) focusing equations. We show how the thermodynamics of trapping horizons is related to these deformation equations in two different formalisms: with and without introducing quasilocal energy. In the formalism with the quasilocal energy, the Hawking mass in four dimension is generalized to higher dimension, and it is found that the deformation of this energy inside a marginal surface can be also decomposed into the contributions from matter fields and gravitational radiation as in the four dimension. In the formalism without the quasilocal energy, we generalize the definition of slowly evolving future outer trapping horizons proposed by Booth to past trapping horizons. The dynamics of the trapping horizons in FLRW universe is given as an example. Especially, the slowly evolving past trapping horizon in the FLRW universe has close relation to the scenario of slow-roll inflation. Up to the second order of the slowly evolving parameter in this generalization, the temperature (surface gravity) associated with the slowly evolving trapping horizon in the FLRW universe is essentially the same as the one defined by using the quasilocal energy.
\end{abstract}

*e-mail address: caolm@phys.kindai.ac.jp, caolm@itp.ac.cn 


\section{Introduction}

Quantum mechanics together with general relativity (GR) predicts that black hole behaves like a black body, emitting thermal radiations, with a temperature proportional to its surface gravity at the black hole horizon and with an entropy proportional to its horizon area [1, 2. The Hawking temperature and the horizon entropy together with the black hole mass obey the first law of black hole thermodynamics 3 . Since these seminal works in the 1970s, the relation among thermodynamics, quantum theory and spacetime geometry has been widely discussed, and recent status can be found in a nice review [4.

Most of the studies of the black hole thermodynamics have been focused on the event horizons of stationary black holes. For example, Kerr-Newmann solution family in GR. However, this kind of horizon strongly depends on the global structure of the spacetime. For example, to define an event horizon, we have to know the future null infinity of the spacetime. The so called apparent horizon defined by Hawking [5] also depends on the slicing (or $3+1$ decomposition) of the spacetime. To classify the two surfaces embedded in some slice (for example, trapped, untrapped and marginal trapped) of the spacetime, of course, one has to study the extrinsic properties of the two surfaces embedded in this slice. To define the apparent horizon (a hypersurface), one has to repeat this classification in each slice of the spacetimes. Recent years, based on the Hawking's definition of the apparent horizon, people have given some definitions of the so called quasilocal horizons, see a review [6]. Further, from a more broad view, some local defined horizon has also been proposed by Jacobson et $a l$, and profound connection between gravitation and thermodynamics has been revealed [7, 8].

The pioneer work on the quasilocal horizon is the trapping horizon defined by Hayward more than ten years ago [9, 10, 11. Roughly speaking, this kind of horizon is a hypersurface foliated by marginal surfaces of the spacetime. However, here, the so called marginal is different from the one given by Hawking: The former is a two dimension surface embedded in the spacetime [12], while the later is a kind of two dimension surface embedded in some slice of the spacetime. The trapping horizon can be null, spacelike or timelike according to different spacetime structures. At the end of 1998, Ashtekar et al proposed a new horizon which is called isolated horizon [13, 14, 15, 16, 17. This kind of horizon is null, and does not exchange matter and energy with it's environment. About two years later, dynamical horizon is also proposed by Ashtekar and Krishnan [18, [19. It's a spacelike hypersurface and dynamically evolves. Another interest object is the so called slowly evolving horizon developed by Booth et al 20, 21, 22. In this proposal, the marginal surface (and associated physical quantities) slowly evolves on the future trapping horizon (In fact, in this theory, one can also consider the event horizon [22]). The slowly evolving horizon describes some near equilibrium state of the thermodynamics of the spacetime.

To study the dynamics of the quasilocal horizon, usually, there are two formalisms: One of them heavily depend on some quasilocal energy inside a given two dimension surface. By using Einstein equation and the quasilocal en- 
ergy, one may directly gets some first law like equation. Actually, to study the dynamics of the trapping horizon in general spherically symmetric spacetime, Hayward has translated the Einstein equation into a simple first law like equation by selecting the quasilocal energy to be Misner-Sharp energy [26. Without the spherical symmetry, the problem becomes complicated. Based on the Hawking mass (energy) [27] and focusing and cross focusing equations, the dynamics of the trapping horizon is also studied by Hayward et al [28, 29, 30, 31. In this formalism, the quasilocal energy plays a key role, while the (cross) focusing equations link the variation of the quasilocal energy, matter fields and some possible gravitational radiation together. Another formalism is independent of the quasilocal energy. In some sense, the most important implement in this method is the (cross) focusing equations (or generalized version) 1 . From these focusing (cross focusing) equations, firstly, one can defines some energy flux which is naturally related to the variation of the area of the cross section of the quasilocal horizon. This is a Clausius like equation if we simply regard that the area of the cross section corresponds to the entropy associated with the horizon. Secondly, one can also study the variation of the angular momentum of the horizon. Further, by assuming the first law of thermodynamics is still valid on the quasilocal horizon, from the Clausiu like equation and the variation of the angular momentum, one finally gets some energy of the horizon. In this formalism, we need not introduce some quasilocal energy in advance. Contrarily, the energy of the horizon can be regarded as a byproduct of the theory [6, 18, 19, 20, 21, 22. However, these two formalism are both useless for the isolated horizon. In fact, there is no dynamical version first law of thermodynamics (or dynamical version Clausius relation) associated with the isolated horizon. To study this object, one has to consider phase space method [6, 13, 14, 15, 16, 17.

So the (cross) focusing equations are very important to study the dynamics of the quasilocal horizons. Actually, to study the dynamics of local Rindler horizons, Jacobson et al also apply the so called Raychaudhuri equation (corresponding to one of the focusing equations) to relate the variation of the area of the horizon and the matter flux [7, 8. Therefore, these equations inevitably appear whenever we hope to study the dynamics of the horizon quasilocally or locally. On the other hand, the (cross) focusing equations can be get from the study of the deformation of the codimension-2 spacelike submanifold. In the light of the importance of these equations, in this paper, we study the deformation of a spacelike submanifold with an arbitrary codimension, and a local frame independent equation will be given. In the case of codimension-1, this equation reduces to the evolution equation of the extrinsic curvature of a spacelike hypersurface in an $n$-dimensional Einstein theory. In the more interesting case of codimension-2, after selecting a local null frame, this equation naturally reduces to the well known (cross) focusing equations.

According to the two formalisms mentioned in previous paragraph, in this paper, we show how the thermodynamics of the trapping horizons are related to these deformation equations in two different approaches: with and without

\footnotetext{
${ }^{1}$ The Einstein equation is always used in any formalism.
} 
introducing the quasilocal energy. In the first formalism, we generalize the Hawking mass (energy) in four dimension to higher dimension and study the deformation of this energy. We find the deformation of this energy inside a marginal surface (More precisely, the evolution of the energy inside the marginal surface on the horizon) can be also decomposed into the contributions from the matter fields and the gravitational radiation as in the case of the four dimension. When the marginal surfaces are closed Einstein manifolds, we also study the first law like equation of the trapping horizon. Roughly speaking, it has a similar form as the one with the spherical symmetry. However, generally, it's impossible to define a surface gravity which is a constant on the marginal surface. Further, the surface gravity also evolves on the trapping horizon even in the spherically symmetric case. This means the system is generally nonequilibrium (even far from some equilibrium point) if we regard the temperature is proportional to the surface gravity. To make the problem easy to understand, it's necessary to study some near equilibrium state at first. The slowly evolving horizon is just the object which describes the near equilibrium state of the horizon thermodynamics. However, most of the studies of the slowly evolving horizon have been focused on the future outer trapping horizons [20, 21, 22, and it's quite necessary to study a slowly evolving past trapping horizon if we hope to study the near equilibrium state of some spacetime which only contains the past trapping horizon (for example, our cosmology). Therefore, in the formalism without introducing the quasilocal energy, we generalize the definition of the slowly evolving future outer trapping horizon to the past trapping horizon.

The dynamics of the trapping horizon in FLRW universe is studied as an example. We find the slowly evolving past trapping horizon in the FLRW universe has close relation to the scenario of the slow-roll inflation. With the slowly evolving conditions, we also find: up to the second order of the corresponding slowly evolving parameter, the temperature (surface gravity) associated with the slowly evolving past trapping horizon in the FLRW universe is essentially the same of the temperature (surface gravity) defined by using the quasilocal energy.

This paper is organized as follows: In Sec.2, a preliminary of submanifold theory is given. In Sec.3, we deduce the deformation equation of a spacelike submanifold with an arbitrary codimension, and the cases of codimension-1 and codimension-2 are given as examples. In Sec.4, By selecting some local frame, we show our equation reduces to the (cross) focusing equations (in the case of codimension-2). We also give the deformation equation of the $S O(1,1)$ connection associated with the local frame. The general definition of the trapping horizon is provided in Sec.5. In Sec.6, we generalize the Hawking mass of the four dimension to the higher dimension, and study the deformation of this quasilocal energy by using the general deformation equation we have obtained. The dynamics of the trapping horizon is discussed based on the deformation of this generalized Hawking energy. In Sec.7, Firstly, we study the null trapping horizon which corresponds to some equilibrium state, and then we generalize the slowly evolving future outer trapping horizon to the past cases. In Sec.8, the tapping horizon in the FLRW universe is investigated. We give the slowly 
evolving conditions of the trapping horizon in the FLRW universe. The surface gravity associated with this kind of trapping horizon is also studied. The Sec.9 is conclusion and discussion.

\section{Theory of Submanifold and Geometry of Codi- mension -2 Surface}

To define the trapping horizon, one has to study the intrinsic and the extrinsic geometries of the codimension-2 spacelike surface, $S$, embedded in the spacetime $(\mathcal{M}, g)$, and then define the union of all the codimension-2 surfaces which satisfy some conditions (be marginal) to be the horizon of the spacetime. So it's important to study the geometry of the codimension- 2 surface.

To describe the geometry of the codimension-2 surface, in this section, we list some important formula in the submanifold theory. We use abstract index notation to make the formula have similar style as the theory of the hypersurface in GR [32]. More details can be found in the papers of Carter [33, 34, 35]. The readers who are familiar with the submanifold theory can skip this subsection.

For a spacelike codimension-2 surface, from the submanifold theory, one can always decompose the metric of the spacetime into

$$
g_{a b}=h_{a b}+q_{a b},
$$

where $q_{a b}$ is the induced metric of the surface $S$. The induced metric $q_{a b}$ is Riemannian because that $S$ is spacelike, while the transverse part, i.e., $h_{a b}$, is Lorentzian. By this decomposition, the corresponding projection operators are given by $q_{a}^{b}$ and $h_{a}^{b}$, and they satisfy

$$
q_{a}^{c} q_{c}^{b}=q_{a}^{b}, \quad h_{a}^{c} h_{c}^{b}=h_{a}^{b}, \quad h_{a}^{c} q_{c}^{b}=0 .
$$

Since $h_{a b}$ is Lorentzian, it's natural to introduce two future directed null vector fields $\ell$ and $n$, and express $h_{a b}$ as

$$
h_{a b}=-\ell_{a} n_{b}-n_{a} \ell_{b}=\varepsilon_{I J} e_{a}^{I} e_{b}^{J} .
$$

where $I$ and $J$ take values $\{1,2\}$, and $e^{1}=\ell, e^{2}=n$. The symbol $\varepsilon_{I J}$ represents a constant matrix given by $\varepsilon_{11}=\varepsilon_{22}=0, \varepsilon_{12}=\varepsilon_{21}=-1$. According to pointing to singularity or not, the vectors $n$ and $\ell$ are called inward (ingoing) and outward (outgoing) respectively. Obviously, there are some freedoms to choose $\ell$ and $n$. However, if we require $\ell_{a} n^{a}=-1$, the only remainder freedom is just the rescaling of the null vectors, i.e., $\ell \rightarrow \lambda \ell, n \rightarrow n / \lambda$ with some positive regular function $\lambda$.

Certainly, one can also introduce an orthogonal frame such that $h_{a b}$ can be expressed as

$$
h_{a b}=-u_{a} u_{b}+v_{a} v_{b},
$$

where $u_{a}$ and $v_{a}$ satisfy: $u^{a} u_{a}=-1, v^{a} v_{a}=1$ and $u^{a} v_{a}=0$. Now, in eq.(3), we can take $e^{1}=u, e^{2}=v, \varepsilon_{11}=-\varepsilon_{22}=-1$ and $\varepsilon_{12}=\varepsilon_{21}=0$. Similar to the 
null frame, there are also some freedoms (for example, the $S O(1,1)$ rotation of the frame) to take different $u_{a}$ and $v_{a}$.

Assuming the covariant derivative of the spacetime $(\mathcal{M}, g)$ is given by $\nabla$, then, second fundamental tensor $K_{a b}^{c}$ is defined as 33 .

$$
K_{a b}^{c}=q_{a}^{d} q_{b}^{e} \nabla_{d} q_{e}^{c} .
$$

This is an important extrinsic quantity of the surface $S$, and it can be defined without introducing any local frame of the spacetime. It is easy to find that the second fundamental tensor satisfies

$$
K_{a b}{ }^{c}=K_{b a}{ }^{c}, \quad h_{a}{ }^{d} K_{d b}{ }^{c}=K_{a b}{ }^{d} q_{d}^{c}=0 .
$$

This tensor can be decomposed into a traceless part $\left(C_{a b}^{c}\right)$ and a trace part $\left(K^{c}\right)$, i.e.,

$$
K_{a b}{ }^{c}=\frac{1}{n-2} q_{a b} K^{c}+C_{a b}^{c},
$$

where $K^{c}=g^{a b} K_{a b}^{c}$ is called extrinsic curvature vector or mean curvature vector, which is an important tensor in the submanifold theory. By using the null frame, one gets these extrinsic quantities along the directions of $\ell$ and $n$ :

$$
K_{a b}^{(\ell)}=-K_{a b}{ }^{c} \ell_{c}=q_{a}{ }^{c} q_{b}{ }^{d} \nabla_{c} \ell_{d}, \quad K_{a b}^{(n)}=-K_{a b}{ }^{c} n_{c}=q_{a}{ }^{c} q_{b}{ }^{d} \nabla_{c} n_{d} .
$$

Similarly, the extrinsic vector is also decomposed as

$$
\theta^{(\ell)}=-K^{c} \ell_{c}=q^{a b} \nabla_{a} \ell_{b}, \quad \theta^{(n)}=-K^{c} n_{c}=q^{a b} \nabla_{a} n_{b} .
$$

These two quantities are called the expansions along $\ell$ and $n$ respectively. The traceless part is decomposed as

$$
\begin{aligned}
& \sigma_{a b}^{(\ell)}=-C_{a b}{ }^{c} \ell_{c}=\left(q_{a}{ }^{c} q_{b}{ }^{d}-\frac{1}{n-2} q_{a b} q^{c d}\right) \nabla_{c} \ell_{d}, \\
& \sigma_{a b}^{(n)}=-C_{a b}{ }^{c} n_{c}=\left(q_{a}{ }^{c} q_{b}{ }^{d}-\frac{1}{n-2} q_{a b} q^{c d}\right) \nabla_{c} n_{d} .
\end{aligned}
$$

These are just the usual shear tensors along the directions of $\ell$ and $n$. For the orthogonal frame $\{u, v\}$, we can also get $K_{a b}^{(u)}=-K_{a b}{ }^{c} u_{c}, K_{a b}^{(v)}=-K_{a b}{ }^{c} v_{c}$ and the corresponding expansions and shear tensors. Actually, for an arbitrary normal vector $X$, we can define

$$
K_{a b}^{(X)}=-K_{a b}^{c} X_{c}=q_{a}^{c} q_{b}^{d} \nabla_{c} X_{d}
$$

and the expansion and the shear tensor are respectively given by

$$
\theta^{(X)}=-K^{c} X_{c}, \quad \sigma_{a b}^{(X)}=-C_{a b}^{c} X_{c} .
$$

To study the intrinsic geometry of $S$, it's necessary to introduce the corresponding connection or covariant derivative $D_{a}$ on $S$. For the tensor field which 
is invariant under projection operator $q_{a}^{b}$, for example, $T_{a}^{b}$, the corresponding covariant derivative is defined by

$$
D_{c} T_{a}^{b}=q_{a}{ }^{d} q_{e}^{b} q_{c}^{f} \nabla_{f} T_{d}{ }^{e},
$$

and more general cases are similar. With this definition of the covariant derivative, for vectors $\xi$ and $\eta$ which are tangent to $S$ (invariant under the projection operator $\left.q_{a}^{b}\right)$, it's easy to find

$$
\eta^{c} \nabla_{c} \xi^{b}=\eta^{c} D_{c} \xi^{b}+K_{a c}{ }^{b} \eta^{a} \xi^{c} .
$$

This is just Gauss's formula. By using this covariant derivative $D_{a}$, from the usual definition

$$
R_{a b c d} \xi^{d}=\left(D_{a} D_{b}-D_{b} D_{a}\right) \xi_{c},
$$

one gets the intrinsic Riemann curvature tensor $R_{a b c d}$. The relation between this intrinsic curvature of $S$ and the curvature of the spacetime is encoded in Gauss equation:

$$
R_{a b c d}=K_{c a}{ }^{e} K_{b d e}-K_{c b}{ }^{e} K_{a d e}+q_{a}{ }^{e} q_{b}{ }^{f} q_{c}{ }^{g} q_{d}{ }^{h} \mathscr{R}_{e f g h},
$$

where $\mathscr{R}_{a b c d}$ is the Riemann curvature of the spacetime. This equation can be easily found from the definitions of $D_{a}$ and $R_{a b c d}$.

Similar to the covariant $D_{a}$ for the intrinsic geometry of $S$, for a mixed tensor $T_{a b c} \ldots$ with tangent indices $a, b$ and a normal index $c$, it's convenient to define a covariant derivative $\tilde{D}_{a}$ as follows:

$$
\tilde{D}_{e} T_{a b c \cdots}=h_{c}{ }^{d} \cdots q_{a}^{g} q_{b}{ }^{h} \cdots q_{e}^{f} \nabla_{f} T_{g h d} .
$$

Obviously, for tangent tensor (which is invariant under $q_{a}^{b}$ ), this covariant derivative reduces to the derivative $D_{a}$. For an arbitrary normal vector $X$ and a tangent vector $\xi$, by using above definition, it's easy to find

$$
\xi^{c} \nabla_{c} X^{b}=-K_{a}^{b} \xi^{a} X^{c}+\xi^{c} \tilde{D}_{c} X^{b} .
$$

This is just Weingarten's formula. So, for normal vectors, $\tilde{D}_{a}$ is just the usual normal covariant derivative. Based on this covariant derivative, by calculating

$$
\Omega_{a b c d} X^{d}=\left(\tilde{D}_{a} \tilde{D}_{b}-\tilde{D}_{b} \tilde{D}_{a}\right) X_{c}
$$

for an arbitrary normal vector $X$, we get the corresponding curvature tensor $\Omega_{a b c d}$, which has form

$$
\Omega_{a b c d}=q_{a}^{e} q_{b}{ }^{f} h_{c}{ }^{g} h_{d}{ }^{h} \mathscr{R}_{e f g h}+K_{a e d} K_{b}{ }^{e}{ }_{c}-K_{b e d} K_{a c}{ }^{e} .
$$

This is Ricci equation. Obviously, this curvature has property of Weyl tensor, in fact, after some rearrangement, it can be expressed as

$$
\Omega_{a b c d}=q_{a}^{e} q_{b}{ }^{f} h_{c}{ }^{g} h_{d}{ }^{h} \mathscr{C}_{\text {efgh }}+C_{a e d} C_{b}{ }^{e}{ }_{c}-C_{b e d} C_{a}{ }^{e}{ }_{c},
$$


where $\mathscr{C}_{e f g h}$ is the Weyl tensor of the spacetime, while $C_{a b c}$ is the traceless part of the second fundamental tensor.

Further, in our codimension-2 cases, from eq.(15), for an arbitrary normal vector $X_{a}=\alpha \ell_{a}+\beta n_{a}$, it's easy to find

$$
\tilde{D}_{a} X_{b}=\left(D_{a} \alpha+\omega_{a} \alpha\right) \ell_{b}+\left(D_{a} \beta-\omega_{a} \beta\right) n_{b},
$$

where $\omega_{a}$ is defined as

$$
\omega_{a}=-q_{a}^{e} n_{d} \nabla_{e} \ell^{d}
$$

This is just the normal covariant derivative given in some references (for example [22]). Sometime, the $\omega_{a}$ is called the $S O(1,1)$ connection of the $S O(1,1)$ normal bundle (see, for example, [23]). Of course, this definition of the connection on the normal bundle depends on the null frame (so it's gauge dependant). Similarly, if we consider the orthogonal frame $\{u, v\}$, for $X_{a}=\alpha u_{a}+\beta v_{a}$, it's easy to find

$$
\tilde{D}_{a} X_{b}=\left(D_{a} \alpha+\omega_{a} \beta\right) u_{b}+\left(D_{a} \beta+\omega_{a} \alpha\right) v_{b},
$$

and now $\omega_{a}$ is defined as

$$
\omega_{a}=-q_{a}{ }^{c} u^{b} \nabla_{c} v_{b} .
$$

It should be noted here: we have used the same notation $\omega_{a}$ as in the case of the null frame, but their values are usually different from each other.

Generally, the connection is defined to be

$$
\omega_{a b c}=\varepsilon_{I J} e_{c}^{I} \tilde{D}_{a} e_{b}^{J}=\omega_{a} \epsilon_{b c},
$$

where $\epsilon_{a b}=n_{a} \ell_{b}-\ell_{a} n_{b}$ for the null frame $\{\ell, n\}$, and $\omega_{a}$ is given in eq.(21). While for the orthogonal frame $\{u, v\}, \epsilon_{a b}=u_{a} v_{b}-v_{a} u_{b}$ and $\omega_{a}$ can be found in eq. (23) (The properties of the tensor $\epsilon_{a b}$ is given in Appendix B.). It's easy find this $\omega_{a b c}$ satisfies standard relations

$$
\omega_{a b c} \ell^{c}=\tilde{D}_{a} \ell_{b}, \quad \omega_{a b c} n^{c}=\tilde{D}_{a} n_{b}
$$

for the null frame, and

$$
\omega_{a b c} u^{c}=\tilde{D}_{a} u_{b}, \quad \omega_{a b c} v^{c}=\tilde{D}_{a} v_{b}
$$

for the orthogonal frame. For the null frame $\{\ell, n\}$ or the orthogonal frame $\{u, v\}$, from eq.(17) and above two relations, it's easy to find that the curvature tensor $\Omega_{a b c d}$ now can be put into

$$
\Omega_{a b c d}=\tilde{D}_{a} \omega_{b c d}-\tilde{D}_{b} \omega_{a c d}+\omega_{a c}^{e} \omega_{b e d}-\omega_{b c}{ }^{e} \omega_{a e d} .
$$

Considering the definition of $\tilde{D}_{a}$, this curvature is just the one proposed by Carter 33, 34, 35]:

$$
\Omega_{a b c d}=\left(h_{c}^{e} h_{d}^{f} q_{a}^{g} q_{b}^{h} \nabla_{g} \omega_{h e f}+\omega_{a c}^{e} \omega_{b e d}\right)-(a \leftrightarrow b)
$$


Actually, the expressions (27) and (28) are valid in the case with an arbitrary codimension. In the special case of codimension- 2 in this paper, after a short calculation, we find

$$
\Omega_{a b c d}=\left(D_{a} \omega_{b}-D_{b} \omega_{a}\right) \epsilon_{c d} .
$$

Here, $\Omega_{a b}=D_{a} \omega_{b}-D_{b} \omega_{a}$ is the curvature associated with the $S O(1,1)$ connection (21).

Another important formula is Codazzi equation. This equation can be obtained from applying the covariant derivative $\tilde{D}_{a}$ on the second fundamental tensor. From equation

$$
\tilde{D}_{d}\left(K_{a b c} X^{c}\right)=\tilde{D}_{d}\left(K_{a b c}\right) X^{c}+K_{a b c} \tilde{D}_{d} X^{c},
$$

and the relation $K_{a b}{ }^{c} X_{c}=-q_{a}^{e} q_{b}^{f} \nabla_{e} X_{f}$, it's not hard to find:

$$
\tilde{D}_{a} K_{b c d}-\tilde{D}_{b} K_{a c d}=-q_{a}{ }^{e} q_{b}{ }^{f} q_{c}{ }^{h} h_{d}^{g} \mathscr{R}_{e f h g} .
$$

This is just the Codazzi equation. For an arbitrary normal vector $Y$, it gives

$$
\left(\frac{n-3}{n-2}\right) D_{a} \theta^{(Y)}-D_{b} \sigma_{a}^{(Y) b}+K_{d} \tilde{D}_{a} Y^{d}-K_{a d}^{b} \tilde{D}_{b} Y^{d}=q_{a}^{e} q^{b c} Y^{d} \mathscr{R}_{e b c d} .
$$

In the case of codimension- 1 , this equation is just the so called momentum constraint equation in Hamiltonian formalism in GR if we select $Y^{a}=u^{a}$, where $u^{a}$ is the unit normal vector of some spacelike hypersurface. In the case of codimension-2, by using eq.(25), immediately, we get

$$
\left(\frac{n-3}{n-2}\right)\left(D_{a}-\omega_{a}\right) \theta^{(\ell)}-\left(D_{b}-\omega_{b}\right) \sigma_{a}^{(\ell) b}=q_{a}^{e} q^{b c} \ell^{d} \mathscr{R}_{e b c d}
$$

and

$$
\left(\frac{n-3}{n-2}\right)\left(D_{a}+\omega_{a}\right) \theta^{(n)}-\left(D_{b}+\omega_{b}\right) \sigma_{a}^{(n) b}=q_{a}^{e} q^{b c} n^{d} \mathscr{R}_{e b c d} .
$$

The right hands of above equations can also be transformed into the form composed by the Weyl tensor $\mathscr{C}_{a b c d}$ and Einstein tensor $\mathscr{G}_{a b}$ :

$$
q_{a}^{e} q^{b c} Y^{d} \mathscr{R}_{e b c d}=q_{a}^{e} q^{b c} Y^{d} \mathscr{C}_{e b c d}-\left(\frac{n-3}{n-2}\right) q_{a}{ }^{e} Y^{b} \mathscr{G}_{e b}
$$

We can get similar equations

$$
\begin{gathered}
\left(\frac{n-3}{n-2}\right)\left(D_{a} \theta^{(u)}-\omega_{a} \theta^{(v)}\right)-\left(D_{b} \sigma_{a}^{(u) b}-\omega_{b} \sigma_{a}^{(v) b}\right)=q_{a}^{e} q^{b c} u^{d} \mathscr{R}_{e b c d} \\
\left(\frac{n-3}{n-2}\right)\left(D_{a} \theta^{(v)}-\omega_{a} \theta^{(u)}\right)-\left(D_{b} \sigma_{a}^{(v) b}-\omega_{b} \sigma_{a}^{(u) b}\right)=q_{a}^{e} q^{b c} v^{d} \mathscr{R}_{e b c d}
\end{gathered}
$$

by using eq.(26) if the orthogonal frame is considered. Eqs.(14), (18) and (31) are important relations in the submanifold theory. They are valid in the case 
with an arbitrary codimension. In the four dimension, some of the formula for the codimension-2 surfaces we have listed here can also be found in 22 . It should be noted here: Some definition might be not well defined if we only consider a single surface. For example, $\nabla_{a}$ is not well defined if the support of the tensor field is confined to the single surface. Actually, in this case, only $q_{a}^{b} \nabla_{b}$ is well defined on the surface [34. However, in this paper, we do not consider this possibility. In our setting, we can always imagine that $S$ is a leaf of the foliation of some neighborhood of $S$. By this consideration, $\nabla_{a}$ is always well defined.

\section{Deformation of Submanifold}

\subsection{Cases with Arbitrary Codimensions}

Now, let's consider the deformation of some spacelike surface $S$ embedded in the spacetime with an arbitrary codimension. Assume $X^{a}$ is a normal vector, then, generally speaking, the Lie derivative of the projection operator $q_{a}^{b}$ along $X^{a}$ is not vanished. However, we can consider a foliation of some neighborhood of $S$ (Certainly, $S$ is a leaf of this foliation), and require that $X$ has some relation to the structure of this foliation such that the projection operator is Lie dragging along $X$, i.e.,

$$
\mathcal{L}_{X}\left(q_{a}^{b}\right)=0
$$

where $\mathcal{L}_{X}$ is the Lie derivative along $X$. This relation just means: any tangent tensor (invariant under $q_{a}^{b}$ ) preserves to be a tangent tensor under the Lie derivative along $X$. For example, for any tangent vector $\xi^{a}$, we have $\mathcal{L}_{X} \xi^{a}=$ $\mathcal{L}_{X}\left(\xi^{b} q_{b}{ }^{a}\right)=q_{b}{ }^{a} \mathcal{L}_{X} \xi^{b}$. So the resulting vector is still a tangent vector. The relation (38) also means that

$$
\mathcal{L}_{X}\left(h_{a}{ }^{b}\right)=0 .
$$

This equation can be easily found from the relation $q_{a}{ }^{b}+h_{a}{ }^{b}=\delta_{a}{ }^{b}$.

Of course, now $X$ is constrained by above two equations (only one of them is independent). The situation is very similar to the $3+1$ decomposition in GR. In that case, for some foliation parameter $\tau$, the evolution vector $X$ is required to satisfy $\mathcal{L}_{X} \tau=1$, and the three dimension projection operator is also Lie dragging along the evolution vector $X$. Here, we have generalized this relation to the case with an arbitrary codimension. For the case of codimension-1, more details can be found in reference [36.

Contrarily, for a given normal vector $X$, we can find an appropriate foliation of some neighborhood of the given codimension-2 surface such that the corresponding projection operator is Lie dragging along $X$.

The relation (38) automatically implies following two equations:

$$
q_{a}^{c} \mathcal{L}_{X} q_{c}^{b}=0, \quad h_{a}^{c} \mathcal{L}_{X} q_{c}^{b}=0 .
$$

After straightforward calculation, the first equation implies

$$
X^{d} q_{a}^{c} \nabla_{d} q_{c b}=-X^{d} h_{b c} \nabla_{d} h_{a}^{c}=h_{b d} q_{a}^{c} \nabla_{c} X^{d}=\tilde{D}_{a} X_{b} .
$$


So, for another normal vector $Y$ (which is arbitrary), we arrive at

$$
q_{a}^{c} X^{d} \nabla_{d} Y_{c}=-Y_{c} \tilde{D}_{a} X^{c} .
$$

This equation just provides the expression of the tangent part of the vector $X^{d} \nabla_{d} Y_{c}$. Generally, it may has a normal part. The second equation in eq. (40) gives result

$$
q_{c}^{b} X^{d} \nabla_{d} q_{a}^{c}=\tilde{D}^{b} X_{a}=-h_{a}^{c} q_{d}^{b} \nabla_{c} X^{d}
$$

where we have used eq.(41). So, by considering the definition of $\tilde{D}_{a}$ in eq.(15), we have

$$
h_{b}{ }^{d} q_{a}^{c}\left(\nabla_{c} X_{d}+\nabla_{d} X_{c}\right)=h_{b}{ }^{d} q_{a}^{c} \mathcal{L}_{X} g_{c d}=0,
$$

This relation just means: after the deformation, the spacetime metric still preserves the orthogonal property of the projection operators. Furthermore, for the (arbitrary) normal vector $Y$, eq.(43) also implies relation

$$
[X, Y]^{e} q_{e b}=0,
$$

This just tells us that the Lie bracket of $X$ and $Y$ is also a normal vector. Actually, if this condition is regarded as a primary assumption, then, one can easily get the equation (42). This logic has been used in the paper by Booth [22]. Now, it's easy to find that equations

$$
q_{b}{ }^{d} q_{a}^{c} \mathcal{L}_{X} q_{c}^{b}=h_{b}{ }^{d} q_{a}^{c} \mathcal{L}_{X} q_{c}^{b}=q_{b}{ }^{d} h_{a}^{c} \mathcal{L}_{X} q_{c}{ }^{b}=h_{b}{ }^{d} h_{a}{ }^{c} \mathcal{L}_{X} q_{c}{ }^{b}=0
$$

are trivially satisfied. So there are no further useful constraint conditions provided by eq.(38).

By using the property that projection operator is Lie dragging along $X$, we can get a simple expression for the second fundamental tensor:

$$
\mathcal{L}_{X} q_{a b}=q_{a}^{c} q_{b}{ }^{d} \mathcal{L}_{X} q_{c d}=-2 K_{a b}{ }^{c} X_{c}=2 K_{a b}^{(X)} .
$$

Similarly, one finds the expansion along $X$ can be expressed as

$$
\mathcal{L}_{X} \epsilon_{q}=\theta^{(X)} \epsilon_{q},
$$

where $\epsilon_{q}$ is the area element of the $(n-2)$-dimension submanifold $S$. Actually, once some quantity is invariant under the projection operator, we can use (38) to simplify the Lie derivative of the quantity as above two examples.

Let's consider the deformation of the second fundamental tensor $K_{a b}^{c}$ (projecting along the direction of $Y$ ). From above discussion, we have

$$
\mathcal{L}_{X} K_{a b}^{(Y)}=q_{a}^{c} q_{b}{ }^{d} \mathcal{L}_{X} K_{c d}^{(Y)}=q_{a}{ }^{c} q_{b}{ }^{d} X^{e} \nabla_{e} K_{c d}^{(Y)}+K_{a}^{(X) c} K_{c b}^{(Y)}+K_{b}^{(X) c} K_{c a}^{(Y)} .
$$

After a little bit complicated calculation (especially for the first term in right hand of above equation), we gets

$$
\begin{aligned}
\mathcal{L}_{X} K_{a b}^{(Y)}= & q_{a}^{c} q_{b}{ }^{d} X^{e} Y^{f} \mathscr{R}_{e c d f}+K_{a}^{(Y) c} K_{b c}^{(X)}-Y^{c} \tilde{D}_{a} \tilde{D}_{b} X_{c} \\
& +K_{a c b}\left(Y_{d} \tilde{D}^{c} X^{d}\right)-K_{a b c}\left(X^{d} \nabla_{d} Y^{c}\right) .
\end{aligned}
$$


The details to get above equation are given in Appendix A. Considering

$$
\left(\mathcal{L}_{X} q^{a c}\right) q_{c b}=-2 q^{a c} K_{c b}^{(X)} \quad \text { and } \quad q_{a}^{c} \mathcal{L}_{X} h_{c b}=0,
$$

we have

$$
\begin{aligned}
\mathcal{L}_{X} \theta^{(Y)}= & \mathcal{L}_{X}\left(K_{a b}^{(Y)} g^{a b}\right)=\left(\mathcal{L}_{X} K_{a b}^{(Y)}\right) g^{a b}+K_{a b}^{(Y)} \mathcal{L}_{X} g^{a b} \\
& =\left(\mathcal{L}_{X} K_{a b}^{(Y)}\right) g^{a b}+K_{a b}^{(Y)} \mathcal{L}_{X}\left(q^{a b}+h^{a b}\right) \\
& =\left(\mathcal{L}_{X} K_{a b}^{(Y)}\right) g^{a b}-2 K_{a b}^{(Y)} K^{(X) a b}
\end{aligned}
$$

After substituting this result into eq.(50), we get the deformation equation of the expansion $\theta^{(Y)}$ (The normal vector $Y$ is arbitrary.),

$$
\mathcal{L}_{X} \theta^{(Y)}=q^{c d} X^{e} Y^{f} \mathscr{R}_{e c d f}-K^{(Y) a b} K_{a b}^{(X)}-Y^{c} \tilde{D}_{a} \tilde{D}^{a} X_{c}-K_{c}\left(X^{d} \nabla_{d} Y^{c}\right) .
$$

The results (501) and (52) are valid in the case with an arbitrary codimension. In fact, in above discussions, we have not impose any requirement on the dimension of the part associated with $h_{a b}$.

In above discussions, we only consider the deformation of the submanifold along a normal vector $X^{a}$. For a tangent vector, for example, $\phi^{a}$, the Lie derivative of $\theta^{(Y)}$ along $\phi^{a}$ is constrained by the Codazzi equations (31) and (32):

$$
\begin{aligned}
& \left(\frac{n-3}{n-2}\right) \mathcal{L}_{\phi} \theta^{(Y)}=\phi^{a} D_{b} \sigma_{a}^{(Y) b}-\left(\frac{n-3}{n-2}\right) \phi^{a} K_{d} \tilde{D}_{a} Y^{d} \\
& +\phi^{a} C_{a d}^{b} \tilde{D}_{b} Y^{d}+q^{f g} \phi^{e} Y^{h} \mathscr{R}_{e f g h} .
\end{aligned}
$$

Eqs.(50) and (52) are main results of this subsection. To understand them, in following two subsections, we will apply them to the cases of codimension- 1 and codimension- 2 respectively.

\subsection{Codimension-1 Cases}

In the case of codimension- 1 , we can set $h_{a b}=-u_{a} u_{b}$, where $u^{a}$ is an unit timelike normal vector of the hypersurface (the observer associated with the vector $u^{a}$ is just the so called Euler observer). So the extrinsic curvature is simply given by $K_{a b c}=K_{a b} u_{c}$. In this case, $X$ is just the evolution vector $X_{a}=N u_{a}$ with lapse function $N$. We can select $Y_{a}=u_{a}$ such that $\theta^{(Y)}$ is given by $\theta^{(Y)}=K=-K^{a} u_{a}$. Remembering the definition of $\tilde{D}_{a}$ in eq.(15), we have

$$
\begin{gathered}
Y^{c} \tilde{D}_{a} \tilde{D}^{a} X_{c}=-D_{a} D^{a} N, \quad K^{(Y) a b} K_{a b}^{(X)}=N K^{a b} K_{a b}, \\
q^{f g} X^{e} Y^{h} \mathscr{R}_{e f g h}=-N \mathscr{R}_{a b} u^{a} u^{b}, \quad K_{c}\left(X^{d} \nabla_{d} Y^{c}\right)=0 .
\end{gathered}
$$

So eq.(52) is transformed into a very familiar form

$$
-\frac{1}{N} \mathcal{L}_{X} K=\mathscr{R}_{a b} u^{a} u^{b}+K^{a b} K_{a b}-\frac{1}{N} D^{a} D_{a} N
$$


This is nothing but the evolution equation of $K$ in the $3+1$ formulism of GR (Actually, now, it's $(n-1)+1$ decomposition of the $n$-dimensional Einstein theory). It should be noted here: Usually, in GR, people use the definition of the extrinsic curvature as $K_{a b}=-q_{a}^{c} q_{b}{ }^{d} \nabla_{c} u_{d}$. So it has a sign difference from our definition. If we use this definition, the minus in front of $\mathcal{L}_{X} K / N$ will disappear. Similarly, we can get the evolution equation of $K_{a b}$ from eq.(50) and the Gauss equation (14):

$$
-\frac{1}{N} \mathcal{L}_{X} K_{a b}=-q_{a}^{c} q_{b}{ }^{d} \mathscr{R}_{c d}+R_{a b}+K K_{a b}-2 K_{a c} K_{b}{ }^{c}-\frac{1}{N} D_{a} D_{b} N .
$$

From this simple example, it's very clear how that the vector $X$ is adapted with the foliation of the neighborhood of the hypersurface such that $q_{a}^{b}$ is Lie dragging along it. Of course, for a different lapse function $N$ (or a different evolution vector $X$ ), one has to consider a different foliation structure of the neighborhood of the hypersurface [36].

\subsection{Codimension-2 Cases}

Let's consider the more interesting case of codimension-2. Noted that in this case we have

$$
\begin{aligned}
& q^{f g} X^{e} Y^{h} \mathscr{R}_{e f g h}=-X^{e} Y^{h} \mathscr{R}_{e h}-h^{f g} X^{e} Y^{h} \mathscr{R}_{e f g h} \\
& =-X^{e} Y^{h} \mathscr{R}_{e h}-\frac{1}{2} h^{f g} h^{e h} \mathscr{R}_{e f g h}\left(X_{b} Y^{b}\right) .
\end{aligned}
$$

However, from the Gauss equation (14), we get

$$
\mathscr{R}-2 \mathscr{R}_{a b} h^{a b}+h^{a c} h^{b d} \mathscr{R}_{a b c d}=R+K_{a b c} K^{a b c}-K^{e} K_{e} .
$$

After substituting eqs.(57) and (58) into eq.(52), we find

$$
\begin{aligned}
\mathcal{L}_{X} \theta^{(Y)}= & -\mathscr{G}_{a b}\left[X^{a} Y^{b}-h^{a b}\left(X_{e} Y^{e}\right)\right]-K^{(Y) a b} K_{a b}^{(X)} \\
& +\frac{1}{2}\left(R+K_{a b c} K^{a b c}-K^{a} K_{a}\right) \cdot\left(X_{e} Y^{e}\right) \\
& -Y^{c} \tilde{D}_{a} \tilde{D}^{a} X_{c}-K_{c}\left(X^{d} \nabla_{d} Y^{c}\right)
\end{aligned}
$$

where $\mathscr{G}_{a b}$ is the Einstein tensor of the spacetime. We can rearrange the terms in above equation, and transform it into another more covariant form

$$
\begin{aligned}
\mathcal{L}_{X} \theta^{(Y)}= & -\left(\mathscr{G}_{a b}+K_{c d a} K^{c d}{ }_{b}\right)\left[X^{a} Y^{b}-h^{a b}\left(X_{e} Y^{e}\right)\right] \\
& +\frac{1}{2}\left(R-K_{a b c} K^{a b c}-K_{c} K^{c}\right) \cdot\left(X_{e} Y^{e}\right) \\
& -Y^{e} \tilde{D}_{c} \tilde{D}^{c} X_{e}-K_{c}\left(X^{e} \nabla_{e} Y^{c}\right) .
\end{aligned}
$$

This is the main result of this subsection. Eq.(60) is very important to study the thermodynamics of the horizons. Of course, here, this deformation equation 
is valid for an arbitrary codimension-2 surface (not only for the case which has closed relation to the horizons).

During above reductions, the local frames $e^{I}=\{\ell, n\}$ or $e^{I}=\{u, v\}$ are not necessary. However, to find the explicit expression for the term $Y^{e} \tilde{D}_{c} \tilde{D}^{c} X_{e}$, it's better to introduce some local frame. After some calculation (see Appendix C), we find

$$
\begin{aligned}
Y^{e} \tilde{D}_{c} \tilde{D}^{c} X_{e} & =\varepsilon_{I J}\left(Y^{I} D^{c} D_{c} X^{J}\right)+2 \varepsilon_{I J}\left(\omega^{c} \epsilon_{b d} Y^{b} e^{I d} D_{c} X^{J}\right) \\
& +D_{c} \omega^{c}\left(\epsilon_{b d} Y^{b} X^{d}\right)+\omega_{c} \omega^{c}\left(X_{e} Y^{e}\right)
\end{aligned}
$$

where $Y^{I}$ is the component of $Y^{a}$ along the vector $e_{a}^{I}$, i.e., $Y^{I}=Y^{a} e_{a}^{I}$. $D_{a}$ is the covariant derivative on the codimension-2 surface. For the null frame $\{\ell, n\}$, the matrix $\varepsilon_{I J}$ and tensor $\epsilon_{a b}$ are given in eqs.(3) and (24) respectively, and the $S O(1,1)$ connection $\omega_{a}$ can be found in eq.(21). While for the orthogonal frame $\{u, v\}, \varepsilon_{I J}$ and $\epsilon_{a b}$ are given just bellow eqs.(4) and (24) respectively, and the connection $\omega_{a}$ can be found in eq.(23).

From above results, it is easy to find that the operator $\mathcal{L}_{X}$ satisfies $\mathcal{L}_{X}+\mathcal{L}_{Z}=$ $\mathcal{L}_{X+Z}$. However, due to the existence of the term $Y^{e} \tilde{D}_{c} \tilde{D}^{c} X_{e}$, it has no property as the usual Lie derivative. For example, the action of the operator on the scalar $\theta^{(Y)}$ generally has property

$$
\mathcal{L}_{(f X)} \theta^{(Y)} \neq f \mathcal{L}_{X} \theta^{(Y)},
$$

where $f$ is a function on the spacetime. This behavior comes from the fact that we require that the projection operator is Lie dragging along $X$. Of course, in the case that $f$ is a constant on the codimension-2 surface, from eq. (61), one finds the operator $\mathcal{L}_{X}$ reduces to the usual Lie derivative. We will find this point in the spherically symmetric cases, for example, the FLRW universe. For $Y$ vector, we have

$$
\mathcal{L}_{X} \theta^{(f Y)}=f \mathcal{L}_{X} \theta^{(Y)}+\theta^{(Y)} \mathcal{L}_{X} f,
$$

This is because that the derivative of the $Y$ variable is first order in eq.(60). To make the problem easy to understand, in next section, we give the explicit form of the deformation equation in some local frames.

The property (62) of the operator $\mathcal{L}_{X}$ is very similar to the operator " $\delta$ " defined by Andersson, Mars and Simon 24, 25. Here, to compare this operator $\delta$ with our approach, we give (the notations are a little bit different) the original definition of $\delta$ [25]: Assume $0 \in I \subset R$ and $X$ be an arbitray normal vector at $S$. Let $\Phi_{X}: S \times I \rightarrow \mathcal{M}$ be a differential map such that for each fixed $\tau \in I, \Phi_{X}(\cdot, \tau)$ is an immersion and for fixed $p \in S, \Phi_{X}(p, \tau)$ is a curve starting at $p$ with tangent vector $X(p)$. This way, a family of codimension-2 surfaces $S_{\tau}=\Phi_{X}(S, \tau)$ is defined. Assume $Y_{\tau}$ be a nowhere zero normal vector of $S_{\tau}$ which is differentiable with respect to $\tau$, let $\theta^{\left(Y_{\tau}\right)}$ be the expansion of $S_{\tau}$ along $Y_{\tau}$, then, the variation of $\theta^{(Y)}$ is defined as $\left.\delta_{X} \theta^{(Y)} \equiv \partial_{\tau} \theta^{\left(Y_{\tau}\right)}\right|_{\tau=0}$. This is a kind of geometric variation and we can also assume that $X$ has a part $X^{\|}$which is 
tangent to $S$. By using this definition, and assuming $X=X^{\|}+A \ell-B n$, one gets [25]:

$$
\begin{aligned}
\delta_{X} \theta^{(\ell)}= & X^{\|}\left(\theta^{(\ell)}\right)+\mathrm{a} \theta^{(\ell)}-\Delta_{S} B+2 \omega^{a} D_{a} B-A\left(K_{a b}^{(\ell)} K^{(\ell) a b}+\mathscr{G}_{a b} \ell^{a} \ell^{b}\right) \\
& +\frac{B}{2}\left(-2 \omega_{a} \omega^{a}+2 D_{a} \omega^{a}+R+K_{a} K^{a}-2 \mathscr{G}_{a b} \ell^{a} n^{b}\right),
\end{aligned}
$$

where we have chosen $Y=\ell$. $\omega_{a}$ is defined in eq.(21). $\Delta_{S}=D^{a} D_{a}$ is the Laplacian on $\left(S, q_{a b}\right)$, and $\mathrm{a}=-\left.n_{a} \partial_{\tau} \ell_{\tau}^{a}\right|_{\tau=0}$. If we change notations as: $X \rightarrow q$, $\ell \rightarrow l, n \rightarrow k / 2, \omega \rightarrow s, \theta^{(\ell)} \rightarrow \theta, K_{a} K^{a} \rightarrow-H^{2}, A \rightarrow b$ and $B \rightarrow u$, then, (64) is just the original result in the Lemma 3.1 in [25].

Our approach is different from the definition of $\delta$ : For an arbitrary normal vector $X$ of $S$, we assume that there is a foliation of some neighborhood of $S$ such that $q_{a}^{b}$ is Lie dragging along $X$. So our operator $\mathcal{L}_{X}$ is just the usual Lie derivative constrained by (38). By this definition, we can get the value of $\mathcal{L}_{X} \theta^{(Y)}$ on $S$ for each $X$. However, it will be found soon in the next section that the result given by $\delta_{X}$ is very similar to our result by $\mathcal{L}_{X}$ (see the discussion bellow eq.(67)). In our approach, the action of $X^{\|}$is encoded in eq.(53). To get the result of $\delta_{X} \theta^{(Y)}$, we need not know the details of the map $\Phi_{X}$. A

different $X$ may correspond a different map $\Phi$. Similarly, to get $\mathcal{L}_{X} \theta^{(Y)}$, we also need not know the details of the foliation structure of some neighborhood of $S$, and a different $X$ corresponds to a different foliation of a (maybe different) neighborhood of $S$.

\section{Deformation Equations with Local Frames}

\subsection{Expressions in Null Frame}

\subsubsection{Focusing and Cross Focusing Equations}

Eqs.(59) and (60) in last section are independent of any local frame. In this subsection, by choosing the null frame $\{\ell, n\}$, we give two important examples. The components of the extrinsic curvature along these two null vectors can be found in eq.(8), and the corresponding expansions and shear tensors are defined in eqs. (9) and (10) respectively.

Firstly, by setting $Y_{a}=\ell_{a}$ and $X_{a}=A \ell_{a}-B n_{a}$, we have $X_{e} Y^{e}=B$. So eqs. (60) and (61) give result

$$
\begin{aligned}
& \mathcal{L}_{X} \theta^{(\ell)}=\kappa_{X} \theta^{(\ell)}-D_{c} D^{c} B+2 \omega^{c} D_{c} B-B\left[\omega_{c} \omega^{c}-D_{c} \omega^{c}+\mathscr{G}_{a b} \ell^{a} n^{b}\right. \\
& \left.-\frac{1}{2} R-\theta^{(\ell)} \theta^{(n)}\right]-A\left[\mathscr{G}_{a b} \ell^{a} \ell^{b}+\sigma_{a b}^{(\ell)} \sigma^{(\ell) a b}+\frac{1}{n-2} \theta^{(\ell)} \theta^{(\ell)}\right] .
\end{aligned}
$$

Here, we have introduced an important quantity - "surface gravity"

$$
\kappa_{X}=-n^{c} X^{e} \nabla_{e} \ell_{c}
$$


and then the last term in eq. (60) becomes

$$
\begin{aligned}
& -K^{c}\left(X^{e} \nabla_{e} \ell_{c}\right)=-K^{d} h_{d}^{c}\left(X^{e} \nabla_{e} \ell_{c}\right) \\
& =K^{d}\left(\ell^{c} n_{d}+n^{c} \ell_{d}\right)\left(X^{e} \nabla_{e} \ell_{c}\right)=\kappa_{X} \theta^{(\ell)} .
\end{aligned}
$$

Since we have not discuss any horizon until now, so, in eq.(66), $\kappa_{X}$ associated with the vector $X$ is not the real surface gravity of the black hole horizon. It should be noted here: if we identify " $\kappa_{X}$ " with "a" in (64), then $\delta_{X} \theta^{(\ell)}$ in (64) is the same of our result (65).

Similarly, by setting $Y_{a}=n_{a}$ and $X_{a}=A \ell_{a}-B n_{a}$, we get

$$
\begin{aligned}
& \mathcal{L}_{X} \theta^{(n)}=-\kappa_{X} \theta^{(n)}+D_{c} D^{c} A+2 \omega^{c} D_{c} A+A\left[\omega_{c} \omega^{c}+D_{c} \omega^{c}+\mathscr{G}_{a b} n^{a} \ell^{b}\right. \\
& \left.-\frac{1}{2} R-\theta^{(\ell)} \theta^{(n)}\right]+B\left[\mathscr{G}_{a b} n^{a} n^{b}+\sigma_{a b}^{(n)} \sigma^{(n) a b}+\frac{1}{n-2} \theta^{(n)} \theta^{(n)}\right] .
\end{aligned}
$$

In the four dimension, eqs. (65) and (68) are just the equations given by Booth 22 . In the case where $A=1, B=0$ (or $A=0, B=-1$ ), eq. (65) (or eq.(68)) is just the so called focusing equation. In the case where $A=0, B=-1$ (or $A=1, B=0$ ), eq.(65) (or eq.(68) ) gives the cross focusing equation (see eqs.(94) in next section) [28, 29, 30].

\subsection{2 $Y$ is dual to $X$}

The second interest example is the case where $X^{e} Y_{e}=0$. Under this requirement, eq. (60) becomes very simple:

$$
\mathcal{L}_{X} \theta^{(Y)}=-\left(\mathscr{G}_{a b}+K_{c d a} K_{b}^{c d}\right) X^{a} Y^{b}-Y^{e} \tilde{D}_{c} \tilde{D}^{c} X_{e}-K_{c}\left(X^{e} \nabla_{e} Y^{c}\right) .
$$

For simplicity, we select $X$ and $Y$ as

$$
X_{a}=A \ell_{a}-B n_{a}, \quad Y_{a}=A \ell_{a}+B n_{a},
$$

where $A$ and $B$ are functions on the spacetime. It should be noted here: above expressions are not the most general forms of $X$ and $Y$ (which satisfy the relation $\left.X_{e} Y^{e}=0\right)$. Actually, here, $X$ and $Y$ are dual to each other, i.e., $Y_{a}=\epsilon_{a b} X^{b}$. The tensor $\epsilon_{a b}$ has been defined in eq.(24). After a short calculation, eq.(61) reduces to

$$
Y^{e} \tilde{D}_{c} \tilde{D}^{c} X_{e}=D_{e}\left(A D^{e} B-B D^{c} A-2 A B \omega^{e}\right) .
$$

It's also easy to find

$$
K_{c}\left(X^{e} \nabla_{e} Y^{c}\right)=-\kappa_{X} \theta^{(X)}-\theta^{(\ell)} \mathcal{L}_{X} A-\theta^{(n)} \mathcal{L}_{X} B .
$$

So the deformation equation of the expansion $\theta^{(Y)}$ becomes

$$
\begin{aligned}
& \mathcal{L}_{X} \theta^{(Y)}=\kappa_{X} \theta^{(X)}-\mathscr{G}_{a b} X^{a} Y^{b}-\sigma_{a b}^{(X)} \sigma^{(Y) a b}-\frac{1}{n-2} \theta^{(X)} \theta^{(Y)} \\
& -D_{e}\left(A D^{e} B-B D^{c} A-2 A B \omega^{e}\right)+\theta^{(\ell)} \mathcal{L}_{X} A+\theta^{(n)} \mathcal{L}_{X} B
\end{aligned}
$$


where $\kappa_{X}$ is defined in eq. (66). In four dimension, by setting $A=1$, this equation is just the one given in $22,38,39]$. Consider eq.(63), we can translate above equation into

$$
\begin{aligned}
& \kappa_{X} \theta^{(X)}=\mathscr{G}_{a b} X^{a} Y^{b}+\sigma_{a b}^{(X)} \sigma^{(Y) a b}+\frac{1}{n-2} \theta^{(X)} \theta^{(Y)} \\
& +D_{e}\left(A D^{e} B-B D^{c} A-2 A B \omega^{e}\right)+A \mathcal{L}_{X} \theta^{(\ell)}+B \mathcal{L}_{X} \theta^{(n)},
\end{aligned}
$$

For $n=4$, this result can also be found in [22, 38, 39]. This is a very important equation to study the thermodynamics of the horizon. If the codimension-2 surface is compact without boundary, then, it's easy to find

$$
\begin{aligned}
\int \kappa_{X} \mathcal{L}_{X} \epsilon_{q}= & \int \epsilon_{q}\left[\mathscr{G}_{a b} X^{a} Y^{b}+\sigma_{a b}^{(X)} \sigma^{(Y) a b}+\frac{1}{n-2} \theta^{(X)} \theta^{(Y)}\right] \\
& +\int \epsilon_{q}\left[A \mathcal{L}_{X} \theta^{(\ell)}+B \mathcal{L}_{X} \theta^{(n)}\right]
\end{aligned}
$$

where $\epsilon_{q}$ is the area element of the codimension-2 surface. Here we have used the relation $\theta^{(X)} \epsilon_{q}=\mathcal{L}_{X} \epsilon_{q}$ in eq.(48).

\subsubsection{Damour-Navier-Stokes like Equation}

The reason to select the null frame $\{\ell, n\}$ is not simply to make the formula easy to understand. In fact, without the local null frame, some important physical quantities can not be defined. For example, $\omega_{a}$ and $\kappa_{X}$, which have close relation to the angular momentum and the surface gravity of the horizon. So another important equation is the deformation equation of $\omega_{a}$. From the definition of $\omega_{a}$ in eq.(21), by using the relations (25) and (42), it's easy to find

$$
\mathcal{L}_{X} \omega_{a}=K_{a c}{ }^{b} \tilde{D}_{b}\left(\epsilon^{c d} X_{d}\right)+D_{a} \kappa_{X}-\frac{1}{2} q_{a}{ }^{b} X^{d} \epsilon^{c e} \mathscr{R}_{d b c e} .
$$

We can also express the right hand of above equation by the Weyl tensor $\mathscr{C}_{\text {abcd }}$ and the Einstein tensor $\mathscr{G}_{a b}$ :

$$
q_{a}{ }^{b} X^{d} \epsilon^{c e} \mathscr{R}_{d b c e}=q_{a}{ }^{b} X^{d} \epsilon^{c e} \mathscr{C}_{d b c e}-\frac{2}{n-2} q_{a}{ }^{b} \epsilon^{c d} X_{d} \mathscr{G}_{b c} .
$$

From the generalized Codazzi equation (32) and eq.(35), we have

$$
\begin{aligned}
& \left(\frac{n-3}{n-2}\right) D_{a} \theta^{(Y)}-D_{c} \sigma_{a}^{(Y) c}+K_{c} \tilde{D}_{a} Y^{c}-K_{a c}{ }^{b} \tilde{D}_{b} Y^{c} \\
& =-q_{a}{ }^{b} h^{c e} Y^{d} \mathscr{C}_{b c e d}-\left(\frac{n-3}{n-2}\right) q_{a}{ }^{b} Y \mathscr{G}_{b c},
\end{aligned}
$$

where $Y_{a}$ is the dual vector of $X_{a}$, i.e., $Y_{a}=\epsilon_{a b} X^{b}$. It's easy to find that

$$
q_{a}^{b} h^{c e} \epsilon^{d f} X_{f} \mathscr{C}_{b c e d}=\frac{1}{2} q_{a}{ }^{b} X^{d} \epsilon^{c e} \mathscr{C}_{d b c e}
$$


always holds. Thus, by combining eqs.(76), (77) and (78), we have

$$
\mathcal{L}_{X} \omega_{a}=D_{a} \kappa_{X}+\left(\frac{n-3}{n-2}\right) D_{a} \theta^{(Y)}-D_{c} \sigma_{a}^{(Y) c}+K_{c} \tilde{D}_{a} Y^{c}+q_{a}^{b} Y^{c} \mathscr{G}_{b c}
$$

The term $K_{c} \tilde{D}_{a} Y^{c}$ can be calculated from eqs.(70) and (20), and above equation becomes

$$
\begin{aligned}
& \mathcal{L}_{X} \omega_{a}+\theta^{(X)} \omega_{a}=D_{a} \kappa_{X}+\left(\frac{n-3}{n-2}\right) D_{a} \theta^{(Y)}-D_{c} \sigma_{a}^{(Y) c} \\
& +q_{a}^{b} Y^{c} \mathscr{G}_{b c}-\theta^{(\ell)} D_{a} A-\theta^{(n)} D_{a} B .
\end{aligned}
$$

In the case where $X$ is self-dual or anti-self-dual, i.e., $X= \pm Y$, by considering the Einstein equation, this equation is a kind of Damour-Navier-Stokes equation 37. In this case, $X$ or $Y$ can be identified to be the evolution vector of the event horizon of the spacetime (Actually, when $X= \pm Y$, eq. (80) can be explained to be the Damour-Navier-Stokes equation on an arbitrary null hypersurface of the spacetime). If eq.800 is applied to the trapping horizons instead of the event horizon, one gets generalized Damour-Navier-Stokes equations [38, 40]. In this case, $X$ is identified to be the evolution vector of the trapping horizon, and $Y$ is just the normal vector of the trapping horizon. Certainly, now, $X$ need not to be self-dual or anti-self-dual, i.e., $X$ need not to be null. More details on the physical meaning of the terms in eq. (80) can be found in the papers by Gourgoulhon et.al. [38, 40,

Let $\phi^{a}$ be a tangent vector, then, we have

$$
\begin{aligned}
& \mathcal{L}_{X} \int \epsilon_{q}\left(\phi^{a} \omega_{a}\right)=\int \epsilon_{q}\left[\theta^{(X)} \phi^{a} \omega_{a}+\mathcal{L}_{X} \phi^{a} \omega_{a}+\phi^{a} \mathcal{L}_{X} \omega_{a}\right] \\
& =\int \epsilon_{q}\left\{\mathcal{L}_{X} \phi^{a} \omega_{a}+\phi^{a}\left[D_{a} \kappa_{X}+\left(\frac{n-3}{n-2}\right) D_{a} \theta^{(Y)}-D_{c} \sigma_{a}^{(Y) c}\right.\right. \\
& \left.\left.+q_{a}{ }^{b} Y^{c} \mathscr{G}_{b c}-\theta^{(\ell)} D_{a} A-\theta^{(n)} D_{a} B\right]\right\}
\end{aligned}
$$

Assume that $\phi^{a}$ also satisfies $\mathcal{L}_{X} \phi^{a}=0$ and $D_{a} \phi^{a}=0$, then we get

$$
\begin{aligned}
\mathcal{L}_{X} \int \epsilon_{q}\left(\phi^{a} \omega_{a}\right)= & \int \epsilon_{q}\left\{\frac{1}{2}\left(D^{a} \phi^{b}+D^{b} \phi^{a}\right) \sigma_{a b}^{(Y)}+\phi^{a} Y^{b} \mathscr{G}_{a b}\right. \\
& \left.+A \phi^{a} D_{a} \theta^{(\ell)}+B \phi^{a} D_{a} \theta^{(n)}\right\}
\end{aligned}
$$

Here, we have assumed that the codimension-2 surface is compact without boundary. It's obvious this equation does not depend on the dimension of the spacetime. The left hand of above equation has closed relation to the angular momentum of the spacetime [41. Actually, the angular momentum can 
be defined as $J_{\phi}=\int \epsilon_{q}\left(\phi^{a} \omega_{a}\right)$. From above equation, it's not hard to find the balance equation of the angular momentum [38.

Eqs.(73) and (80) have important applications in the theory of membrane paradigm 42,43 . Recent progress on this topic can be found in [38, 44, 45, 46] and references therein.

\subsection{Expressions in Orthogonal Frame}

Besides the null frame $\{\ell, n\}$, sometime, it's useful to express the deformation equations in the orthogonal frame $\{u, v\}$. For example, in the studies of the dynamical horizon, it's convenient to choose some orthogonal frame. Assume $X_{a}=A u_{a}+B v_{a}$, and $Y_{a}=u_{a}$, then we have

$$
K_{c}\left(X^{e} \nabla_{e} Y^{c}\right)=-\theta^{(v)} \kappa_{X} .
$$

Similar to the "surface gravity" defined in eq.(66), here, we have defined

$$
\kappa_{X}=v_{c} X^{e} \nabla_{e} u^{c} .
$$

From eq.(61), it's also easy to find

$$
Y^{e} \tilde{D}_{c} \tilde{D}^{c} X_{e}=-D^{c} D_{c} A-2 \omega^{c} D_{c} B-B D_{c} \omega^{c}-A \omega_{c} \omega^{c} .
$$

Substituting above results into eq. (60), we get

$$
\begin{aligned}
& \mathcal{L}_{X} \theta^{(u)}=\theta^{(v)} \kappa_{X}+D^{c} D_{c} A+2 \omega^{c} D_{c} B+B D_{c} \omega^{c} \\
& -B\left(\mathscr{G}_{a b} v^{a} u^{b}+K_{a b}^{(u)} K^{(v) a b}\right)+A\left[\omega_{c} \omega^{c}-\mathscr{G}_{a b} v^{a} v^{b}\right. \\
& \left.-\frac{1}{2}\left(R+K_{a b}^{(u)} K^{(u) a b}+K_{a b}^{(v)} K^{(v) a b}+\theta^{(u)} \theta^{(u)}-\theta^{(v)} \theta^{(v)}\right)\right] .
\end{aligned}
$$

By similar calculation, for $Y_{a}=v_{a}$, we have

$$
\begin{aligned}
& \mathcal{L}_{X} \theta^{(v)}=\theta^{(u)} \kappa_{X}-D^{c} D_{c} B-2 \omega^{c} D_{c} A-A D_{c} \omega^{c} \\
& -A\left(\mathscr{G}_{a b} u^{a} v^{b}+K_{a b}^{(u)} K^{(v) a b}\right)-B\left[\omega_{c} \omega^{c}+\mathscr{G}_{a b} u^{a} u^{b}\right. \\
& \left.-\frac{1}{2}\left(R-K_{a b}^{(u)} K^{(u) a b}-K_{a b}^{(v)} K^{(v) a b}+\theta^{(u)} \theta^{(u)}-\theta^{(v)} \theta^{(v)}\right)\right] .
\end{aligned}
$$

Let $X_{a}=A u_{a}+B v_{a}$ and $Y_{a}=B u_{a}+A v_{a}$, then, $Y$ is the dual vector of $X$, and they automatically satisfy $X^{e} Y_{e}=0$. It's easy to find

$$
\begin{aligned}
\mathcal{L}_{X} \theta^{(Y)}= & \kappa_{X} \theta^{(X)}-D_{c}\left[A D^{c} B-B D^{c} A+\omega^{c}\left(A^{2}-B^{2}\right)\right] \\
& -\mathscr{G}_{a b} X^{a} Y^{b}-\sigma_{a b}^{(X)} \sigma^{(Y) a b}-\frac{1}{n-2} \theta^{(X)} \theta^{(Y)} \\
& +\theta^{(u)} \mathcal{L}_{X} B+\theta^{(v)} \mathcal{L}_{X} A .
\end{aligned}
$$

Considering the relation (63), we have

$$
\mathcal{L}_{X} \theta^{(Y)}=A \mathcal{L}_{X} \theta^{(v)}+B \mathcal{L}_{X} \theta^{(u)}+\theta^{(v)} \mathcal{L}_{X} A+\theta^{(u)} \mathcal{L}_{X} B,
$$


then, when the codimension- 2 surface is compact without boundary, we get

$$
\begin{aligned}
\int \kappa_{X} \mathcal{L}_{X} \epsilon_{q}= & \int \epsilon_{q}\left[\mathscr{G}_{a b} X^{a} Y^{b}+\sigma_{a b}^{(X)} \sigma^{(Y) a b}+\frac{1}{n-2} \theta^{(X)} \theta^{(Y)}\right. \\
& \left.+A \mathcal{L}_{X} \theta^{(v)}+B \mathcal{L}_{X} \theta^{(u)}\right] .
\end{aligned}
$$

We can also get the deformation equation of $\omega_{a}$, which is given by

$$
\mathcal{L}_{X} \omega_{a}=D_{a} \kappa_{X}+\left(\frac{n-3}{n-2}\right) D_{a} \theta^{(Y)}-D_{c} \sigma_{a}^{(Y) c}+K_{c} \tilde{D}_{a} Y^{c}+q_{a}^{b} Y^{c} \mathscr{G}_{b c} .
$$

However, it should be noted here: now, $\omega_{a}$ and $\kappa_{X}$ are given in eqs. (23) and (84) respectively. Although the definitions of $\omega_{a}$ and $\kappa_{X}$ are dependent of the selection of the local frames, the deformation equation of $\omega_{a}$ has similar form in different frames if we give an appropriate definition of the "surface gravity" $\kappa_{X}$. Actually, from above equation or eq.(179), the combination $\left(\mathcal{L}_{X} \omega_{a}-D_{a} \kappa_{X}\right)$ is frame independent.

From the assumption $X_{a}=A u_{a}+B v_{a}, Y_{a}=B u_{a}+A v_{a}$, and eq.(22), we can easily get the explicit expression of the term $K_{c} \tilde{D}_{a} Y^{c}$ :

$$
K_{c} \tilde{D}_{a} Y^{c}=-D_{a} B \theta^{(u)}-D_{a} A \theta^{(v)}-\theta^{(X)} \omega_{a} .
$$

By this, the deformation equation (90) becomes

$$
\begin{aligned}
& \mathcal{L}_{X} \omega_{a}+\theta^{(X)} \omega_{a}=D_{a} \kappa_{X}+\left(\frac{n-3}{n-2}\right) D_{a} \theta^{(Y)}-D_{c} \sigma_{a}^{(Y) c} \\
& +q_{a}^{b} Y^{c} \mathscr{G}_{b c}-\theta^{(u)} D_{a} B-\theta^{(v)} D_{a} A .
\end{aligned}
$$

Similar to get eq.(82), it's not hard to find: for $D_{a} \phi^{a}=0$ and $\mathcal{L}_{X} \phi^{a}=0$, we have

$$
\begin{aligned}
\mathcal{L}_{X} \int \epsilon_{q}\left(\phi^{a} \omega_{a}\right)= & \int \epsilon_{q}\left\{\frac{1}{2}\left(D^{a} \phi^{b}+D^{b} \phi^{a}\right) \sigma_{a b}^{(Y)}+\phi^{a} Y^{b} \mathscr{G}_{a b}\right. \\
& \left.+A \phi^{a} D_{a} \theta^{(v)}+B \phi^{a} D_{a} \theta^{(u)}\right\} .
\end{aligned}
$$

Certainly, to get above equation, compactness of the codimension-2 surface is also required, and this equation has closed relation to the balance equation of the angular momentum of the spacetime.

At the end of this section, we give some discussions:

(i). The so called (cross) focusing equations are just the special examples of eq. (60). Principally, for a pair of arbitrary normal vectors $X$ and $Y$, we can get the value of $\mathcal{L}_{X} \theta^{(Y)}$ on some given codimension-2 surface according to eq. (60). Of course, in this procedure, we have to consider a foliation of some neighborhood of the codimension-2 surface such that the projection operator is Lie dragging along $X$. 
(ii). By selecting different frames, the deformation equations of $\omega_{a}$ 's have similar forms as given in eqs.(79) and (90). Further, the combination $\mathcal{L}_{X} \omega_{a}-$ $D_{a} \kappa_{X}$ is frame independent. To get this conclusion, we have to define an appropriate "surface gravity" $\kappa_{X}$ to match the definition of the $S O(1,1)$ connection $\omega_{a}$. When $X$ is self-dual or anti-self-dual, i.e., $Y= \pm X$, we get the DamourNavier-Stokes equation.

(iii). The situation where $Y$ is the dual of $X$ is very simple and special. The compactness of the codimension-2 surface is important. Without this assumption, we can not get the simple expression for the integrals of the deformation equations, i.e. eqs. (75) and (82) (or eqs.(89) and (93) in the orthogonal frame).

(iv). Until now, we haven't discussed some special hypersurface of the spacetime. In fact, we have focused on the codimension- 2 surfaces. Of course the horizon of the spacetime is kind of hypersurface. In next section, we will find how to study this kind of hypersurface by our knowledge on the geometry of the codimension-2 surface.

\section{Trapping Horizon}

In black hole theory, one of the most important objects is the so called event horizon which is the boundary of the causal past of the future null infinity [5, 32. So, to describe the event horizon, one has to know some global information of the spacetime, for example, the future null infinity. This kind of horizon is a null hypersurface of the spacetime. However, the so called trapping horizon is very different from the event horizon [9, 10, 11. The definition of the trapping horizon is quasilocal, and it does not depend on the asymptotical behavior of the spacetime. This kind of horizon can be null, spacelike or timelike according to different spacetime structures which are involved.

The codimension-2 spacelike surface with $\theta^{(\ell)} \theta^{(n)}=0$ is called marginal surface. The surface with $\theta^{(\ell)} \theta^{(n)}>0$ is called trapped, and $\theta^{(\ell)} \theta^{(n)}<0$ is called untrapped.

By the definitions of the trapped surface and the untrapped surface, we can define two regions in the spacetime: A trapped (untrapped) region is the union of all trapped (untrapped) surfaces.

We can give similar definitions by using the extrinsic curvature vector $K^{a}$ from the relation $K^{c} K_{c}=-2 \theta^{(\ell)} \theta^{(n)}$. Sometime, the formulism without the null frame is enough. However, to give a detailed study of the marginal surfaces, for example, to give a classification of the marginal surfaces, it's inevitable to introduce the null frame or some similar structure.

A marginal surface is called future if $\theta^{(\ell)}=0, \theta^{(n)}<0$. In this case, if $\mathcal{L}_{n} \theta^{(\ell)}<0$, we call the future marginal surface is outer. The future marginal surface with $\mathcal{L}_{n} \theta^{(\ell)}>0$ is called inner.

The past marginal surface is defined by $\theta^{(n)}=0, \theta^{(\ell)}>0$. Similarly, the past marginal surface with $\mathcal{L}_{\ell} \theta^{(n)}>0$ is called outer, and the case with $\mathcal{L}_{\ell} \theta^{(n)}<0$ is called inner.

In some neighborhood of a given codimension- 2 surface, we can imaging this 
codimension-2 surface belongs to a foliation of this neighborhood such that the projection operators are Lie dragging along the null vector $\ell$. This way, we can calculate $\mathcal{L}_{\ell} \theta^{(Y)}$ for an arbitrary normal vector $Y$. By the similar assumption and calculation, we get $\mathcal{L}_{n} \theta^{(Y)}$. By this consideration, we get the values for $\mathcal{L}_{\ell} \theta^{(\ell)}, \mathcal{L}_{\ell} \theta^{(n)}, \mathcal{L}_{n} \theta^{(\ell)}$ and $\mathcal{L}_{n} \theta^{(n)}$ on the given codimension-2 surface (Of course, this surface belongs to the different foliations of some neighborhoods according to the different deformation vector $X^{\prime}$ 's). Actually, similar to get eqs.(65) and (68), it's easy to find the (cross) focusing equations:

$$
\begin{aligned}
\mathcal{L}_{\ell} \theta^{(\ell)} & =\kappa_{\ell} \theta^{(\ell)}-\mathscr{G}_{a b} \ell^{a} \ell^{b}-\sigma_{a b}^{(\ell)} \sigma^{(\ell) a b}-\frac{1}{n-2} \theta^{(\ell)} \theta^{(\ell)} \\
\mathcal{L}_{n} \theta^{(n)} & =-\kappa_{n} \theta^{(n)}-\mathscr{G}_{a b} n^{a} n^{b}-\sigma_{a b}^{(n)} \sigma^{(n) a b}-\frac{1}{n-2} \theta^{(n)} \theta^{(n)} \\
\mathcal{L}_{n} \theta^{(\ell)} & =\kappa_{n} \theta^{(\ell)}+\omega_{c} \omega^{c}-D_{c} \omega^{c}+\mathscr{G}_{a b} \ell^{a} n^{b}-\frac{1}{2} R-\theta^{(\ell)} \theta^{(n)} \\
\mathcal{L}_{\ell} \theta^{(n)} & =-\kappa_{\ell} \theta^{(n)}+\omega_{c} \omega^{c}+D_{c} \omega^{c}+\mathscr{G}_{a b} n^{a} \ell^{b}-\frac{1}{2} R-\theta^{(\ell)} \theta^{(n)}
\end{aligned}
$$

So, for the future marginal surface, the classification of the outer and inner is determined by

$$
\mathcal{L}_{n} \theta^{(\ell)}=\omega_{c} \omega^{c}-D_{c} \omega^{c}+\mathscr{G}_{a b} \ell^{a} n^{b}-\frac{1}{2} R
$$

on the future marginal surface, while for the past marginal surface, this kind of classification is determined by the value

$$
\mathcal{L}_{\ell} \theta^{(n)}=\omega_{c} \omega^{c}+D_{c} \omega^{c}+\mathscr{G}_{a b} \ell^{a} n^{b}-\frac{1}{2} R
$$

on the past marginal surface. Assuming the marginal surface is closed, then, for the future outer marginal surface and the past inner marginal surface, above relations give strong constraints on the scalar curvature $R$ of the marginal surface. For example, $\int \epsilon_{q} R$ should be positive if some energy condition is imposed.

The so called trapping horizon is the closure of a hypersurface foliated by the marginal surfaces. The classification of the trapping horizon inherits from the classification of the marginal surfaces [9, 10, 11.

So we can imagine that the trapping horizon $\mathcal{H}$ is a hypersurface which is foliated by a family of $(n-2)$-dimensional marginal surfaces $S_{\tau}$, where the $\tau \in R$ is called foliation parameter of the trapping horizon. Assume $X$ is the so called "evolution" vector, i.e., the vector which is tangent to $\mathcal{H}$ and normal to $S_{\tau}$ and satisfies $\mathcal{L}_{X} \tau=1$. The details can be found in, for example, reference [38]. Since $X$ is normal to the codimension- 2 surface $S_{\tau}$, it can be expressed as $X_{a}=A \ell_{a}-B n_{a}$ as before. Of course, if we require that the projection operator is Lie dragging along $X$, then $X$ is also a deformation vector. In this case, all the formula in previous sections can be used for this $X$. In following discussion, we always assume that the evolution vector $X$ is also a deformation vector.

Here, it should be noted that the foliation near some marginal surface $S_{\tau}$ has been given in advance. For instance, the neighborhood of $S_{\tau}$ characterized 
by the foliation parameter from $\tau-\Delta \tau$ to $\tau+\Delta \tau$ is given a priori (and the foliation structure of this neighborhood is also provided simultaneously). By requiring that $q_{a}^{b}$ is Lie dragging along $X$, we get the value of $\mathcal{L}_{X} \theta^{(Y)}$ on $S_{\tau}$. For another vector, for example, $\ell$, we have no a priori neighborhood of $S_{\tau}$ and the associated foliation structure. However, we can get the value of $\mathcal{L}_{\ell} \theta^{(Y)}$ on $S_{\tau}$ by requiring that $q_{a}^{b}$ is Lie dragging along $\ell$ although the corresponding neighborhood and associated foliation structure is not provided explicitly. This point has been discussed at the end of Sec.3.

For the evolution vector $X$, some discussions are listed in order:

(i). For the reparameter or the relabeling of the foliation $\tau \rightarrow \tau^{\prime}(\tau)$, the evolution vector changes as $X \rightarrow X^{\prime}=\left(d \tau^{\prime} / d \tau\right)^{-1} X$. As we have pointed out before: According to eq. (60), the relation between $\mathcal{L}_{X} \theta^{(Y)}$ and $\mathcal{L}_{X^{\prime}} \theta^{(Y)}$ is complicated due to the existence of the term $Y^{c} \tilde{D}_{a} \tilde{D}^{a} X_{c}$. So generally we have no $\mathcal{L}_{X^{\prime}} \theta^{(Y)}=f \mathcal{L}_{X} \theta^{(Y)}$ if $X^{\prime}=f X$ (i.e., eq.(62)). However, since that $\tau$ and $\tau^{\prime}=\tau^{\prime}(\tau)$ are both constants on the marginal surfaces, we really have $\mathcal{L}_{X^{\prime}} \theta^{(Y)}=\left(d \tau^{\prime} / d \tau\right)^{-1} \mathcal{L}_{X} \theta^{(Y)}$. Thus, this kind of reparameter does not effect our discussion (the classification of the trapping horizons).

(ii). It's easy to find that the position of the marginal surface is independent of the rescaling of the null frame $\{\ell, n\} \rightarrow\{\lambda \ell, n / \lambda\}$ with some positive regular function $\lambda$. However, the value of $\mathcal{L}_{n} \theta^{(\ell)}\left(\mathcal{L}_{\ell} \theta^{(n)}\right)$ on the future (past) trapping horizon really depends on the rescaling of the null frame. So the classification of the outer and inner of the trapping horizons is complicated. However, in the case with enough symmetries, the classification of outer of inner of the trapping horizon is independent of the rescaling of the null frame.

(iii). One can not naively get $\mathcal{L}_{X} \theta^{(\ell)}$ just from the linear combination of $\mathcal{L}_{\ell} \theta^{(\ell)}$ and $\mathcal{L}_{n} \theta^{(\ell)}$ listed in eq.(94). The reason is very clear according to eq.(62). The values of $\mathcal{L}_{X} \theta^{(\ell)}$ and $\mathcal{L}_{X} \theta^{(n)}$ are given in eqs. (65) and (68). Certainly, for some special case (for example, the case of spherically symmetric spacetime), $\mathcal{L}_{X} \theta^{(\ell)}$ is really a linear combination of $\mathcal{L}_{\ell} \theta^{(\ell)}$ and $\mathcal{L}_{n} \theta^{(\ell)}$.

(iv). Since the trapping horizon is a hypersurface foliated by the marginal surfaces, generally we have $\theta^{(\ell)}=0$ on this hypersurface. Further, since that the "evolution" vector $X$ is tangent to this hypersurface, then we expect $\mathcal{L}_{X} \theta^{(\ell)}=0$ on this hypersurface. From eq.(65), we get a complicated differential equation of the function $B$. So it's difficult to get the explicit expression of $X$. However, in some cases with enough symmetries (for example, spherically symmetric spacetime), the problem becomes very simple. We can find that $X$ can be fixed up to a function which does not depend on the points of the codimension- 2 surface. This will become clear in the FLRW universe.

\section{Horizon Dynamics with Quasilocal Energy}

To study the dynamics of the horizon, we can firstly assume some quasilocal energy, i.e., the energy inside the closed codimension-2 surfaces, and then study the deformation of this energy. Generally speaking, the deformation of the energy can be reduced into a form like the first law of thermodynamics if this 
quasilocal energy is appropriately selected. Need not to say, this discussion heavily depends on the definition of the quasilocal energy.

\subsection{General Spherically Symmetric Cases}

The results in previous sections are valid for any spacetime. In this subsection, as an example, we will discuss the spherically symmetric spacetime. Here, in this simple case, we will not introduce the quasilocal energy at first. Instead, we show how the deformation equation of the expansion can be transformed into an interest form where the quasilocal energy will appear naturally. Generally, the metric of an $n$-dimensional spherically symmetric spacetime can be written as

$$
g=\beta_{\mu \nu}(y) d y^{\mu} d y^{\nu}+r(y)^{2} \gamma_{i j}(z) d z^{i} d z^{j},
$$

where $\gamma_{i j} d z^{i} d z^{j}$ is the standard metric of an $(n-2)$-dimension sphere of radius one. Assuming the codimension-2 surface is just the $(n-2)$-sphere, then we have

$$
h_{a b} d x^{a} d x^{b}=\beta_{\mu \nu} d y^{\mu} d y^{\nu}, \quad q_{a b} d x^{a} d x^{b}=r^{2} \gamma_{i j} d z^{i} d z^{j} .
$$

With these identifications, it's easy to find

$$
K_{a b c}=-\frac{1}{r} q_{a b} \nabla_{c} r=\frac{1}{n-2} q_{a b} K_{c}, \quad K_{c}=-\frac{(n-2)}{r} \nabla_{c} r .
$$

Assuming $X$ and $Y$ are two normal vectors, so, from $q_{a b} X^{b}=q_{a b} Y^{b}=0$, we get that $X_{a} d x^{a}=X_{\mu} d y^{\mu}$ and $Y_{a} d x^{a}=Y_{\mu} d y^{\mu}$, where $X_{\mu}$ and $Y_{\mu}$ are components of $X$ and $Y$ in coordinate bases. Further, we require that $X$ satisfies $\mathcal{L}_{X} q_{a}{ }^{b}=0$. A short calculation shows that $X_{\mu}$ only depends on the coordinates $y^{\mu}$. Thus, according to the definition of $\tilde{D}_{a}$, it's easy to find

$$
\tilde{D}_{a} X_{b}=q_{a}{ }^{c} h_{b}{ }^{d} \nabla_{c} X_{d}=0 .
$$

So the two order derivative term of $X$ in eq. 600 is vanishing, and the behavior of the usual Lie derivative of $X$ in eq.(62) is restored. This means that eq. (600) only depends on the direction of $X$ (but not the norm of $X$ ). Now, we have $\mathcal{L}_{X} \theta^{(Y)}=X^{a} \nabla_{a} \theta^{(Y)}$, and considering $\theta^{(Y)}=-K_{c} Y^{c}$, we get

$$
\mathcal{L}_{X} \theta^{(Y)}=X^{e} \nabla_{e} Y^{c}\left(\frac{n-2}{r} \nabla_{c} r\right)+\frac{n-2}{r} Y^{c} X^{e} \nabla_{e} \nabla_{c} r-\frac{n-2}{r^{2}} X^{e} Y^{c} \nabla_{e} r \nabla_{c} r .
$$

Substituting above result and eq.(99) into eq.(60), we have

$$
\begin{aligned}
& \left(\frac{n-2}{r}\right) X^{a} Y^{b} \nabla_{a} \nabla_{b} r-\frac{(n-2)(n-3)}{2 r^{2}}\left[1-\nabla_{a} r \nabla^{a} r\right]\left(X^{e} Y_{e}\right) \\
& =-\mathscr{G}_{a b}\left[X^{a} Y^{b}-h^{a b}\left(X^{e} Y_{e}\right)\right] .
\end{aligned}
$$

Here, we have substituted the value of the scalar curvature of the sphere: $R=(n-2)(n-3) / r^{2}$. Now, let's choose $X$ just be the extrinsic curvature vector (or mean curvature vector) of the sphere, i.e., $X_{a}=K_{a}=-(n-2) \nabla_{a} r / r$. 
Obviously, this selection satisfies the condition that the components of the deformation vector $X$ are only functions of the coordinates $y$. Considering

$$
\nabla_{b}\left(\nabla^{a} r \nabla_{a} r\right)=\nabla^{a} r \nabla_{b} \nabla_{a} r+\left(\nabla_{b} \nabla_{a} r\right) \nabla^{a} r=2 \nabla^{a} r \nabla_{b} \nabla_{a} r
$$

and rearranging the terms in eq.(102), we get

$$
\frac{(n-2)}{2 r^{n-2}} Y^{b} \nabla_{b}\left[r^{n-3}\left(1-\nabla_{a} r \nabla^{a} r\right)\right]=\mathscr{G}_{a b}\left[\nabla^{a} r Y^{b}-h^{a b}\left(\nabla^{e} r Y_{e}\right)\right] .
$$

After integrating this equation on the codimension-2 surface, we get

$$
Y^{b} \nabla_{b}\left[\frac{(n-2) \Omega_{n-2}}{16 \pi G} r^{n-3}\left(1-\nabla_{a} r \nabla^{a} r\right)\right]=\mathscr{A}_{\mathscr{T}_{a b}}\left[\nabla^{a} r Y^{b}-h^{a b}\left(\nabla^{e} r Y_{e}\right)\right],
$$

where $\mathscr{A}=\Omega_{n-2} r^{n-2}$ is the area of a sphere of radius $r$, and $\mathscr{T}_{a b}$ is the energy momentum tensor of the spacetime. Here, we have used the Einstein equation $\mathscr{G}_{a b}=8 \pi G \mathscr{T}_{a b}$. By defining

$$
\mathscr{E}=\frac{(n-2) \Omega_{n-2}}{16 \pi G} r^{n-3}\left(1-\nabla_{a} r \nabla^{a} r\right),
$$

and

$$
\psi_{a}=\mathscr{T}_{a b} \nabla^{b} r+w \nabla_{a} r, \quad w=-\frac{1}{2} h^{a b} \mathscr{T}_{a b},
$$

we can put eq.(105) into a very familiar form:

$$
\mathcal{L}_{Y} \mathscr{E}=\mathscr{A} \psi_{a} Y^{a}+w \mathcal{L}_{Y} \mathscr{V},
$$

where the normal vector $Y$ is arbitrary. $\mathscr{V}$ is the volume inside the $(n-2)$ sphere which is given by $\mathscr{V}=\Omega_{n-2} r^{n-1} /(n-1)$. It should be noted: we have selected a special $X$ which is proportional to the mean curvature vector of the $(n-2)$-sphere. So we are considering a special foliation of some neighborhood of the codimension- 2 surface. By this selection of the deformation vector, the deformation equation (60) can be transformed into above simple form.

The quantity $\mathscr{E}$ actually is just the so called Misner-Sharp energy insider the $(n-2)$-sphere [26. While $\psi_{a}$ is a kind of energy flux which is called energy supply. The scalar $w$ is an energy density, and $w \mathcal{L}_{Y} \mathscr{V}$ in eq. (108) is a work term. Eq. (108) is called unified first law, which is firstly found by Hayward in GR 9, [10, 11. Certainly, one can get eq.(108) directly from the Einstein equation. Here, we have deduced it from the deformation equation of the codimension-2 surface.

Above discussion is independent of the null frame. If we introduce the null frame $\{\ell, n\}$, then the mean curvature vector can be expressed as

$$
K_{a}=\theta^{(\ell)} n_{a}+\theta^{(n)} \ell_{a}=-\frac{(n-2)}{r} \nabla_{a} r,
$$

and other quantities can also be expressed by using the null frame, for example, the Misner-Sharp energy $\mathscr{E}$ :

$$
\mathscr{E}=\frac{(n-2) \Omega_{n-2}}{16 \pi G} r^{n-3}\left[1+\frac{2 r^{2}}{(n-2)^{2}} \theta^{(\ell)} \theta^{(n)}\right] .
$$


Certainly, it's also easy to get the expression for the energy supply in this null frame.

Everything is simple when the spherical symmetry exists. However, for the general case, the situation becomes complicated. Maybe, the most difficult problem is: how to select an appropriate quasilocal energy. In the four dimension, to get an equation which is similar to the unified first law (108), Hayward et.al. have used the so called "Hawking mass" [28, 29, 30, 31. In the next subsection, we will give a similar discussion in the higher dimension. However, firstly, we have to generalize the Hawking mass (energy) to the higher dimension $(n \geq 4)$.

\subsection{More General Cases in Higher Dimensions}

Eq.(110) depends on the null frame. However, we can put it into another form

$$
\mathscr{E}=\frac{\Omega_{n-2}}{16 \pi G(n-3)} r^{n-1}\left[R-\left(\frac{n-3}{n-2}\right) K^{c} K_{c}\right],
$$

where $R$ is the scalar curvature of the codimension- 2 surface. This equation still depends on the coordinate function $r$. By using the area, $\int \epsilon_{q}$, of the closed codimension-2 surface, we can transform it into a more general form:

$$
\mathscr{E}=\frac{\left(\int \epsilon_{q}\right)^{\frac{n-3}{n-2}}}{16 \pi G\left(\Omega_{n-2}\right)^{\frac{1}{n-2}}(n-3)}\left\{\frac{\int \epsilon_{q} R}{\left(\int \epsilon_{q}\right)^{\frac{n-4}{n-2}}}-\left(\frac{n-3}{n-2}\right) \frac{\int \epsilon_{q} K_{c} K^{c}}{\left(\int \epsilon_{q}\right)^{\frac{n-4}{n-2}}}\right\} .
$$

Now, $\mathscr{E}$ does not depend on any applied structure of the codimension-2 surface. In static case, a similar mass function has been studied in reference [4] by using the $(n-1)+1$ decomposition of the Einstein theory in $n$-dimension. The energy expression (112) is interesting:

(i). In the four dimension, this energy is nothing but the so called Hawking mass (energy) [27.

(ii). In the general spherically symmetric cases, it reduces to the MisnerSharp energy in the higher dimension (The generalized Minsner-Sharp energy in general Lovelock gravity theory can be found in [51, 52]).

(iii). The term $\left(\int \epsilon_{q} R\right) /\left(\int \epsilon_{q}\right)^{\frac{n-4}{n-2}}$ has close relation to Yamabe invariant (see the chapter 4 of [53] for the details of the Yamabe invariant). For $n=4$, this term is just Euler number, and its variation vanishes. On the other hand, for $n \geq 4$, an arbitrary variation of this term also vanishes if the codimension-2 surface is a compact Einstein manifold without boundary. This can be found as follows: For example, we just take the variation to be $\mathcal{L}_{X}$, i.e., the deformation operator with a deformation vector $X$, then, it's easy to find

$$
\begin{aligned}
& \mathcal{L}_{X} \int \epsilon_{q} R=\int \epsilon_{q}\left\{\left(\frac{n-4}{n-2}\right) R \theta^{(X)}\right. \\
& \left.+2\left[D_{a} D_{b} \sigma^{(X) a b}-\left(\frac{n-3}{n-2}\right) D_{a} D^{a} \theta^{(X)}\right]\right\} .
\end{aligned}
$$


To simplify the expression, let's introduce $\mathscr{A}=\int \epsilon_{q}$. Assuming the codimension2 surface is closed, we get

$$
\mathcal{L}_{X}\left(\frac{\int \epsilon_{q} R}{\mathscr{A}^{\frac{n-4}{n-2}}}\right)=-\left(\frac{n-4}{n-2}\right) \mathscr{A}^{-\frac{n-4}{n-2}} \int \epsilon_{q}\left\{R\left(\frac{\mathcal{L}_{X} \mathscr{A}}{\mathscr{A}}+K^{e} X_{e}\right)\right\},
$$

where $\mathcal{L}_{X} \mathscr{A}=\mathcal{L}_{X} \int \epsilon_{q}=-\int \epsilon_{q}\left(K^{e} X_{e}\right)$. Thus, there are three ways to set above variation to be vanished:

(a). Obviously, the right hand of above equation is identically vanished in the four dimension because that $\int \epsilon_{q} R /\left(\int \epsilon_{q}\right)^{\frac{n-4}{n-2}}$ is just the Euler number of some two dimensional closed manifold.

(b). If the codimension-2 surface is assumed to be a closed Einstein manifold ( $R$ is a constant), then we also get vanished variation.

(c). Selecting a special deformation vector $X$ such that $K^{a} X_{a}$ is a constant on the codimension-2 surface, then we have $\mathcal{L}_{X} \mathscr{A} / \mathscr{A}+K^{e} X_{e}=0$.

To simplify the discussion, here, we introduce a quantity

$$
\mathscr{K}=\frac{1}{16 \pi G\left(\Omega_{n-2}\right)^{\frac{1}{n-2}}(n-3)}\left(\frac{\int \epsilon_{q} R}{\left(\int \epsilon_{q}\right)^{\frac{n-4}{n-2}}}\right) .
$$

Once one of above three conditions is satisfied, we have $\mathcal{L}_{X} \mathscr{K}=0$. Of course, generally, $\mathcal{L}_{X} \mathscr{K} \neq 0$ when $n>4$.

From the definition (112), the deformation of this energy along the normal vector $X$ is given by

$$
\mathcal{L}_{X} \mathscr{E}=\left(\frac{n-3}{n-2}\right)\left(\frac{\mathscr{E}}{\mathscr{A}}\right) \mathcal{L}_{X} \mathscr{A}+\mathscr{A}^{\frac{n-3}{n-2}} \mathcal{L}_{X}\left(\frac{\mathscr{E}}{\mathscr{A}^{\frac{n-3}{n-2}}}\right)
$$

To get this equation, the requirement $\mathcal{L}_{X} \mathscr{K}=0$ is not necessary. However, when $\mathcal{L}_{X} \mathscr{K}=0$, we can transform above equation into another useful form

$$
\mathcal{L}_{X} \mathscr{E}=\left(\frac{n-3}{n-2}\right)\left(\frac{\mathscr{E}}{\mathscr{A}}\right) \mathcal{L}_{X} \mathscr{A}+\mathscr{A}^{\frac{n-3}{n-2}} \mathcal{L}_{X}\left(\frac{\mathscr{E}}{\mathscr{A}^{\frac{n-3}{n-2}}}-\mathscr{K}\right)
$$

After inserting $\mathscr{K}$, the term inside the last bracket in above equation is proportional to $K^{c} K_{c}$.

To find $\psi_{a}$ and $w$ like quantities as in the spherically symmetric case, we need further calculations of the deformation of this energy. Firstly, it's easy to find

$$
\begin{aligned}
& -\mathcal{L}_{X}\left(K^{c} K_{c}\right)=\mathcal{L}_{X} \theta^{(K)} \\
& =-\left(\mathscr{G}_{a b}+K_{c d a} K^{c d}{ }_{b}\right)\left[K^{a} X^{b}-h^{a b}\left(K_{e} X^{e}\right)\right] \\
& +\frac{1}{2}\left(R-K_{a b c} K^{a b c}-K_{c} K^{c}\right) \cdot\left(K_{e} X^{e}\right) \\
& -K^{e} \tilde{D}_{c} \tilde{D}^{c} X_{e}-K_{c}\left(X^{e} \nabla_{e} K^{c}\right),
\end{aligned}
$$


then, we get

$$
\begin{aligned}
& -\frac{1}{2} \mathcal{L}_{X}\left(K^{c} K_{c}\right)=-K^{e} \tilde{D}_{c} \tilde{D}^{c} X_{e}+\left(\frac{1}{2} \mathscr{G}_{a b} h^{a b}\right) \cdot\left(K_{e} X^{e}\right) \\
& -\left(\mathscr{G}_{a b}+C_{c d a} C_{b}^{c d}\right)\left[K^{a} X^{b}-\frac{1}{2} h^{a b}\left(K_{e} X^{e}\right)\right] \\
& \quad+\frac{1}{2}\left[R-\left(\frac{n-1}{n-2}\right) K_{c} K^{c}\right] \cdot\left(K_{e} X^{e}\right),
\end{aligned}
$$

where we have used the fact that $\mathcal{L}_{X}\left(K^{c} K_{c}\right)=2 K_{c}\left(X^{e} \nabla_{e} K^{c}\right)$ and the definition of $C_{a b}{ }^{c}$ in eq.(17). Substituting this result into eq.(116), after some algebraic calculations, we get an important formula of this section:

$$
\begin{aligned}
& \mathcal{L}_{X} \mathscr{E}=\int \epsilon_{q}\left\{\left(\frac{\mathcal{E}}{n-2}\right)\left(\frac{\mathcal{L}_{X} \mathscr{A}}{\mathscr{A}}+K_{e} X^{e}\right)\right\} \\
& +\frac{1}{8 \pi G}\left(\frac{L}{n-2}\right) \int \epsilon_{q}\left\{-K^{e} \tilde{D}_{c} \tilde{D}^{c} X_{e}\right. \\
& -\left(\mathscr{G}_{a b}+C_{c d a} C^{c d}\right)\left[K^{a} X^{b}-\frac{1}{2} h^{a b}\left(K_{e} X^{e}\right)\right] \\
& \left.+\frac{1}{2}\left(\mathscr{G}_{a b} h^{a b}\right) \cdot\left(K_{e} X^{e}\right)\right\},
\end{aligned}
$$

where $L=\mathscr{A}^{\frac{1}{n-2}} /\left(\Omega_{n-2}\right)^{\frac{1}{n-2}}$, and

$$
\mathcal{E}=\frac{L}{16 \pi G(n-3)}\left[R-\left(\frac{n-3}{n-2}\right) K_{c} K^{c}\right] .
$$

Here, $\mathcal{E}$ is a quantity like an energy density. In fact, from the definition of the energy $\mathscr{E}$, we have $\mathscr{E}=\int \epsilon_{q} \mathcal{E}$. Eq. (120) gives the deformation of the energy $\mathscr{E}$ inside the closed codimension -2 surface. Some remarks are listed in order:

(i). If $n=4$, the energy $\mathscr{E}$ is the Hawking mass, and eq. (120) reduces to the one given by Bray et.al 31. Of course, we can also consider the cases with cosmological constant as in 31. According to previous discussion, in this case, we have $\mathcal{L}_{X} \mathscr{K}=0$ because $\mathscr{K}$ is actually a topological quantity now. However, we have to point out: The four dimension is not so special if we put the deformation of the energy into the form (120).

(ii). In the general spherically symmetric cases, $C_{a b}{ }^{c}$ and $K^{e} \tilde{D}_{c} \tilde{D}^{c} X_{e}$ are vanishing. Further, the first term in the right hand of eq. (120) is also vanished, then eq.(120) reduces to the one given in eq.105). This is an expectable result.

(iii). It should be noted that the vector $X$ can be changed arbitrarily in this equation. So, if we select $X$ such that $K^{e} X_{e}=0$, then we get

$$
\mathcal{L}_{X} \mathscr{E}=-\frac{1}{8 \pi G}\left(\frac{L}{n-2}\right) \int \epsilon_{q}\left\{\left(K^{e} \tilde{D}_{c} \tilde{D}^{c} X_{e}\right.\right.
$$




$$
\left.\left.+\mathscr{G}_{a b}+C_{c d a} C_{b}^{c d}\right) K^{a} X^{b}\right\}
$$

Further, when $X$ is just the dual vector of $K_{c}$, then, $K^{c} X_{c}$ automatically vanishes and the term $K^{e} \tilde{D}_{c} \tilde{D}^{c} X_{e}$ is a total derivative on the closed codimension-2 surface (see eq.(171)). So, in this case, the term $K^{e} \tilde{D}_{c} \tilde{D}^{c} X_{e}$ can be omitted.

(iv). It's easy to find: the first term in the right hand of eq.1120 vanishes if $\mathcal{E}$ is a constant on the codimension- 2 surface. This means, on the equi- $\mathcal{E}$ surface, we always have

$$
\begin{aligned}
\mathcal{L}_{X} \mathscr{E}= & \frac{1}{8 \pi G}\left(\frac{L}{n-2}\right) \times \int \epsilon_{q}\left\{-K^{e} \tilde{D}_{c} \tilde{D}^{c} X_{e}\right. \\
& -\left(\mathscr{G}_{a b}+C_{c d a} C^{c d}{ }_{b}\right)\left[K^{a} X^{b}-\frac{1}{2} h^{a b}\left(K_{e} X^{e}\right)\right] \\
& \left.+\frac{1}{2}\left(\mathscr{G}_{a b} h^{a b}\right) \cdot\left(K_{e} X^{e}\right)\right\} .
\end{aligned}
$$

However, unfortunately, the marginal surface (on which we have $K_{c} K^{c}=$ 0 ) is generally not an equi- $\mathcal{E}$ surface unless it is an Einstein manifold. Of course, we also have $\mathcal{L}_{X} \mathscr{K}=0$ if the codimension-2 surface is an Einstein manifold.

(v). We can get the result (123) by another method: Tuning the vector $X$ such that $K_{c} X^{c}$ is always a constant on the codimension-2 surface. By this selection, we have $\mathcal{L}_{X} \mathscr{K}=0$. The requirement in the item (iii) $\left(K_{c} X^{c}=0\right)$ is just a special case of this method.

(vi). It's interest to study the monotonicity of this energy $\mathscr{E}$ as in [31, 47. Although $\mathscr{K}$ is not a topological term for $n>4$, the deformation of the energy $\mathscr{E}$, i.e., eq.(120), really has a very similar structure as in the four dimension. So, it's possible to get some monotonicity behavior of this energy. However, this is far beyond the aims of this paper, and we will not give further discussions here.

Above discussions tell us: The situation becomes a little bit complicated when the codimension- 2 surfaces are not Einstein manifolds. In the following discussion, to simplify the problem, we will firstly discuss the case in which the Einstein condition is imposed. Since the $(n-2)$-dimension Einstein manifolds are always constant curvature spaces when $n<6$, so, in lower dimensions, the generalized Hawking mass (112) has no big difference from the Misner-Sharp Energy in the spherically symmetric cases. However, the problem becomes interesting when $n \geq 6$ because in these cases the Einstein manifolds may be inhomogeneous. To discuss the situation without the Einstein condition on the codimension-2 surface, we have to consider some special deformation vector $X$. We will study this situation at the end of the next subsection. 


\subsection{Dynamics of Trapping Horizon}

The discussion in previous two subsections have given enough preliminaries to study the dynamics of the trapping horizon. Firstly, let's consider the general spherically symmetric cases.

\subsubsection{Spacetime with Spherical Symmetry}

In previous sections, with the spherical symmetry, we have shown that the deformation equation can be transformed into the form of eq.(108). By choosing $X$ (This corresponds to the selection of $Y$ in eq.(108) ) to be the tangent vector of the trapping horizon on which $\nabla_{a} r \nabla^{a} r=0$. More precisely, we choose $X$ to be the evolution vector of the trapping horizon. Then, on the trapping horizon, we have $\mathcal{L}_{X}\left(\nabla_{a} r \nabla^{a} r\right)=0$, so eq.1105) becomes

$$
\mathcal{L}_{X} \mathscr{E}=\left(\frac{\bar{\kappa}}{2 \pi}\right) \mathcal{L}_{X} S
$$

where

$$
\frac{\bar{\kappa}}{2 \pi}=\frac{(n-3)}{4 \pi r}, \quad S=\frac{\mathscr{A}}{4 G} .
$$

This $\bar{\kappa}$ is just the "effective surface gravity" studied in 6, 61. Another interesting surface gravity is defined by

$$
\mathscr{A} \psi_{a} X^{a}=\left(\frac{\kappa}{2 \pi}\right) \mathcal{L}_{X} S
$$

which has a form

$$
\frac{\kappa}{2 \pi}=\frac{4 G}{n-2}\left[\left(\frac{n-3}{\Omega_{n-2}}\right) \frac{\mathscr{E}}{r^{n-2}}-w r\right] .
$$

In above expression of the surface gravity $\kappa$, the energy $\mathscr{E}$ takes value on the trapping horizon. In the four dimension, this surface gravity is just the one defined by Hayward [9]. By this $\kappa$, the evolution of $\mathscr{E}$ on the trapping horizon becomes

$$
\mathcal{L}_{X} \mathscr{E}=\left(\frac{\kappa}{2 \pi}\right) \mathcal{L}_{X} S+w \mathcal{L}_{X} \mathscr{V}
$$

This is a first law like equation. Obviously, $\kappa$ is a constant on the marginal surface $\left(D_{a} \kappa=0\right)$. However, generally, it's not a constant on the full trapping horizon. The reason is: generally, we have no $\mathcal{L}_{X} \kappa=0$. So $\kappa$ may evolve on the trapping horizon.

\subsubsection{General Cases with Einstein Condition}

In the more general cases where the codimension- 2 surface is Einstein (so $\mathcal{L}_{X} \mathscr{K}=$ 0 ), we have eqs.(117), (120) and (123) which have been found in previous sections. Eq. (117) is simple. If we set $X$ to be the evolution vector of the trapping horizon, then we get

$$
\mathcal{L}_{X} \mathscr{E}=\frac{\bar{\kappa}}{2 \pi} \mathcal{L}_{X} S
$$


where the "effective surface gravity" $\bar{\kappa}$ and the entropy $S$ are defined as

$$
\frac{\bar{\kappa}}{2 \pi}=4 G\left(\frac{n-3}{n-2}\right)\left(\frac{\mathscr{E}}{\mathscr{A}}\right), \quad S=\frac{\mathscr{A}}{4 G} .
$$

Of course, this kind of surface gravity is effective, and usually it can not reduce to the surface gravity of the corresponding stationary spacetime. By this definition, it's easy to find $(n-3) \mathscr{E}=(n-2) \bar{T} S$ with $\bar{T}=\bar{\kappa} / 2 \pi[61$.

For the marginal surface, we have $K^{c} K_{c}=0$, so eq.(120) reduces to eq.(123). This is a kind of energy balance equation. Actually, from the Einstein equation $\mathscr{G}_{a b}=8 \pi G \mathscr{T}_{a b}$, eq.(123) becomes

$$
\begin{aligned}
& \mathcal{L}_{X} \mathscr{E}=\left(\frac{L}{n-2}\right) \times \int \epsilon_{q}\left\{-\frac{1}{8 \pi G} K^{e} \tilde{D}_{c} \tilde{D}^{c} X_{e}\right. \\
& -\left(\mathscr{T}_{a b}+\frac{1}{8 \pi G} C_{c d a} C^{c d}{ }_{b}\right)\left[K^{a} X^{b}-\frac{1}{2} h^{a b}\left(K_{e} X^{e}\right)\right] \\
& \left.+\frac{1}{2}\left(\mathscr{T}_{a b} h^{a b}\right) \cdot\left(K_{e} X^{e}\right)\right\} .
\end{aligned}
$$

In the general spherically symmetric cases, in above equation, the terms related to the energy-momentum tensor $\mathscr{T}_{a b}$ give $A \psi_{a} X^{a}+w \mathcal{L}_{X} \mathscr{V}$. However, in the general cases, there are two additional terms corresponding to $C_{a b}{ }^{c}$ and $K^{e} \tilde{D}_{c} \tilde{D}^{c} X_{e}$. Of course, without enough symmetry, it's also not easy to complete the integral, and we can only write the contribution of the energymomentum tensor $\mathscr{T}_{a b}$ into an integral form

$$
\int \epsilon_{q}\left(\psi_{a}^{(m)}+w^{(m)} H_{a}\right) X^{a},
$$

where $w^{(m)}$ and $\psi^{(m)}$ are defined as

$$
w^{(m)}=-\frac{1}{2} \mathscr{T}_{a b} h^{a b}, \quad \psi_{a}^{(m)}=\mathscr{T}_{a b} H^{b}+w^{(m)} H_{a} .
$$

Obviously, $w^{(m)}$ is the same as the one in the spherically symmetric case. The vector $H^{a}$ plays the role of $\nabla^{a} r$ in the spherically symmetric case, which is defined as 2

$$
H_{a}=-\frac{L}{n-2} K_{a},
$$

Eq.(132) comes from the matter fields. While the terms represented by $C_{a b}{ }^{c}$ and $K^{e} \tilde{D}_{c} \tilde{D}^{c} X_{e}$ can be understood as the contribution from the change of a gravitational field. In some sense, we can understand them to be some gravitational radiation. It's obvious that shear tensor $C_{a b}{ }^{c}$ provides an energy supply

$$
\psi_{a}^{(g)} X^{a}=\frac{1}{8 \pi G} C_{c d a} C_{b}^{c d}\left[H^{a} X^{b}-\frac{1}{2} h^{a b}\left(H_{e} X^{e}\right)\right]
$$

\footnotetext{
${ }^{2} \mathrm{By}$ using the normal vector $H^{a}$, we can also define a generalized Kodama vector as: $\epsilon_{a b} H^{b}[48$. In the spherically symmetric case, it's possible to define the surface gravity from this vector, for example, see reference 52 .
} 
as the usual energy-momentum tensor $\mathscr{T}_{a b}$. However, it has no contribution to $w$. Instead, it seems that the term $K^{e} \tilde{D}_{c} \tilde{D}^{c} X_{e}$ plays the role of $w$. Actually, if $K^{e} X_{e}$ is nonvanishing, formally we can define

$$
w^{(g)}=\frac{1}{8 \pi G}\left(\frac{K^{e} \tilde{D}_{c} \tilde{D}^{c} X_{e}}{K^{b} X_{b}}\right)
$$

With these identifications, the contribution of the gravitational radiation is

$$
\int \epsilon_{q}\left(\psi_{a}^{(g)}+w^{(g)} H_{a}\right) X^{a}
$$

So the deformation of the energy $\mathscr{E}$ becomes

$$
\mathcal{L}_{X} \mathscr{E}=\int \epsilon_{q}\left\{\left(\psi_{a}^{(m)}+\psi_{a}^{(g)}\right)+\left(w^{(m)}+w^{(g)}\right) H_{a}\right\} X^{a} .
$$

Similar to eq.(126), we hope the energy supply could provide a similar definition of some surface gravity as the one in eq.(127). Assuming $X$ to be the evolution vector of the trapping horizon, and using eq.(129), we get

$$
\begin{aligned}
& \int \epsilon_{q}\left\{\left(\psi_{a}^{(m)}+\psi_{a}^{(g)}\right) X^{a}\right\}=-\int \epsilon_{q}\left\{\left[\left(\frac{n-3}{n-2}\right)\left(\frac{\mathscr{E}}{\mathscr{A}}\right)\right.\right. \\
& \left.\left.-\left(\frac{L}{n-2}\right)\left(w^{(m)}+w^{(g)}\right)\right] \cdot\left(K_{a} X^{a}\right)\right\} .
\end{aligned}
$$

Unlike the case with the spherical symmetry, usually, we can not get a surface gravity which is a constant on the marginal surface. Actually, by defining

$$
\frac{\kappa}{2 \pi}=\frac{4 G}{n-2}\left[(n-3)\left(\frac{\mathscr{E}}{\mathscr{A}}\right)-L\left(w^{(m)}+w^{(g)}\right)\right]
$$

and considering $\mathcal{L}_{X} \epsilon_{q}=\theta^{(X)} \epsilon_{q}=-\left(K^{a} X_{a}\right) \epsilon_{q}$, at most we have

$$
\int \epsilon_{q}\left\{\left(\psi_{a}^{(m)}+\psi_{a}^{(g)}\right) X^{a}\right\}=\int \frac{\kappa}{2 \pi} \mathcal{L}_{X} s,
$$

where $s=\epsilon_{q} / 4 G$ can be simply understood as the entropy associated with the area element of the marginal surface. Obviously, the surface gravity (140) reduces to the one in eq. (127) if the spherical symmetry is restored. So eq. (138) becomes

$$
\mathcal{L}_{X} \mathscr{E}=\int\left(\frac{\kappa}{2 \pi}\right) \mathcal{L}_{X} s+\int w \mathcal{L}_{X} v
$$

where $w=w^{(m)}+w^{(g)}$ and $\mathcal{L}_{X} v=\theta^{(X)} \epsilon_{q} L /(n-2)$. In the spherically symmetric case, above equation reduces to eq.(128).

Above discussions are independent of any local frame. It's also clear that above description does not depend on the relabeling of the foliation of the trapping horizon ( $X \rightarrow f X$ with some relabeling factor $f$ ). Since only $K_{c} K^{c}=0$ and $\mathcal{L}_{X}\left(K_{c} K^{c}\right)=0$ (on the trapping horizon) have been used, so all discussions are valid on any kind of trapping horizon (future or past, outer or inner). 


\subsubsection{Expression in Null Frame}

However, to make the problem easy to understand, it's better to introduce some null frame. For an arbitrary null frame $\{\ell, n\}$ with $\ell^{a} n_{a}=-1$, we can express $X$ and $K$ to be $X^{a}=A \ell^{a}-B n^{a}$ and $K^{a}=\theta^{(\ell)} n^{a}+\theta^{(n)} \ell^{a}$ respectively. It's easy to find $K^{a} X_{a}=-A \theta^{(\ell)}+B \theta^{(n)}$. From eq.(61), we get

$$
\begin{gathered}
K^{e} \tilde{D}_{c} \tilde{D}^{c} X_{e}=-\theta^{(\ell)}\left(D^{c} D_{c} A+2 \omega^{c} D_{c} A+A D_{c} \omega^{c}+A \omega_{c} \omega^{c}\right) \\
+\theta^{(n)}\left(D^{c} D_{c} B-2 \omega^{c} D_{c} B-B D_{c} \omega^{c}+B \omega_{c} \omega^{c}\right) .
\end{gathered}
$$

So, on the future trapping horizon $\left(\theta^{(\ell)}=0\right.$ and $\theta^{(n)}<0$ ), when $K^{a} X_{a} \neq 0$, we have

$$
\frac{K^{e} \tilde{D}_{c} \tilde{D}^{c} X_{e}}{K^{a} X_{a}}=\frac{1}{B}\left(D^{c} D_{c} B-2 \omega^{c} D_{c} B-B D_{c} \omega^{c}+B \omega_{c} \omega^{c}\right) .
$$

Then, eq.(136) becomes

$$
w^{(g)}=\frac{1}{8 \pi G}\left(\frac{1}{B}\right)\left(D^{c} D_{c} B-2 \omega^{c} D_{c} B-B D_{c} \omega^{c}+B \omega_{c} \omega^{c}\right) .
$$

In the null frame, the energy supply (135) is also very simple:

$$
\psi_{a}^{(g)} X^{a}=-\frac{1}{8 \pi G}\left(\frac{L}{n-2}\right) A \theta^{(n)} \sigma_{a b}^{(\ell)} \sigma^{(\ell) a b} .
$$

Similarly, the energy supply of the matter fields becomes

$$
\psi_{a}^{(m)} X^{a}=-\left(\frac{L}{n-2}\right) A \theta^{(n)} \mathscr{T}_{a b} \ell^{a} \ell^{b} .
$$

After substituting above expressions into eq.(138), we get

$$
\begin{aligned}
& \mathcal{L}_{X} \mathscr{E}=-\left(\frac{L}{n-2}\right) \int \epsilon_{q}\left\{\theta ^ { ( n ) } \left[A\left(\mathscr{T}_{a b} \ell^{a} \ell^{b}+\frac{1}{8 \pi G} \sigma_{a b}^{(\ell)} \sigma^{(\ell) a b}\right)\right.\right. \\
& \left.\left.+B \mathscr{T}_{a b} \ell^{a} n^{b}+\frac{1}{8 \pi G}\left(D^{c} D_{c} B-2 \omega^{c} D_{c} B-B D_{c} \omega^{c}+B \omega_{c} \omega^{c}\right)\right]\right\} .
\end{aligned}
$$

This result is valid even in the case where $K^{a} X_{a}=0$. If $K^{a} X_{a}=0$, we can't define $w^{(g)}$ as eq. (136). Actually, now, the deformation of the energy reduces to eq.(122). Further, since $K^{a} X_{a}=B \theta^{(n)}$ on the future trapping horizon, so, to require that $K^{a} X_{a}=0$, we have to set $X^{a}=A \ell^{a}$ (i.e., the trapping horizon is null). Thus, eq.(143) implies that $K^{e} \tilde{D}_{c} \tilde{D}^{c} X_{e}=0$. Therefore, in the null frame which just has been used, eq.(122) becomes

$$
\mathcal{L}_{X} \mathscr{E}=-\left(\frac{L}{n-2}\right) \int \epsilon_{q} \theta^{(n)}\left[A\left(\mathscr{T}_{a b} \ell^{a} \ell^{b}+\frac{1}{8 \pi G} \sigma_{a b}^{(\ell)} \sigma^{(\ell) a b}\right)\right] .
$$


Obviously, we can get this result just by setting $B=0$ in eq.(148).

Generally, the evolution vector $X$ has to satisfy $\mathcal{L}_{X} \theta^{(\ell)}=0$ on the future trapping horizon. Then, considering eq. (65) and $K^{a} X_{a}=B \theta^{(n)}$, the relation

(148) becomes $\mathcal{L}_{X} \mathscr{E}=\left(\frac{n-3}{n-2}\right)\left(\frac{\mathscr{E}}{\mathscr{A}}\right) \mathcal{L}_{X} \mathscr{A}$. This is nothing but eq. (117) taking value on the trapping horizon. This result implies that the energy $\mathscr{E}$ does not evolve on the null trapping horizon on which we have $K^{a} X_{a}=B \theta^{(n)}=0$. This point can also be directly found from eqs.(65) and (149).

Above discussion is valid for any null frame $\{\ell, n\}$ which satisfies the relation $\ell^{a} n_{a}=-1$. Sometime, one can rescale the null frame $\{\ell, n\}$ such that $\theta^{(n)}$ satisfies [20, 22, 21]

$$
\theta^{(n)}=-\frac{n-2}{L}<0 .
$$

It's easy to find this requirement also means $\mathcal{L}_{n} L=-1$ on the marginal surface of the trapping horizon. Now, eq.(148) is very simple:

$$
\mathcal{L}_{X} \mathscr{E}=\int \epsilon_{q}\left[A\left(\mathscr{T}_{a b} \ell^{a} \ell^{b}+\frac{1}{8 \pi G} \sigma_{a b}^{(\ell)} \sigma^{(\ell) a b}\right)+B\left(\mathscr{T}_{a b} \ell^{a} n^{b}+\frac{\zeta_{a} \zeta^{a}}{8 \pi G}\right)\right] .
$$

where $\zeta_{c}=\omega_{c}-D_{c} \ln B$. Since the codimension-2 surface is closed, all possible total derivatives can be omitted. So after setting $\theta^{(n)}=-(n-2) / L$, we can omit the total derivative terms in eq.(148). By this consideration, eq.(149) also becomes simple:

$$
\mathcal{L}_{X} \mathscr{E}=\int \epsilon_{q}\left[A\left(\mathscr{T}_{a b} \ell^{a} \ell^{b}+\frac{1}{8 \pi G} \sigma_{a b}^{(\ell)} \sigma^{(\ell) a b}\right)\right] .
$$

However, it should be noted here: After selecting this kind of null frame, we can not get result (152) just by setting $B=0$ in eq.(151). This is because there are two $D_{a} \ln B$ terms in eq.(151).

In this null frame, eq.(151) tells us that the evolution of the energy $\mathscr{E}$ on the future trapping horizon is decomposed into two pars:

(i). The contribution of the usual matter fields $-\mathscr{T}_{a b} \ell^{a} \ell^{a}$ and $\mathscr{T}_{a b} \ell^{a} n^{a}$;

(ii). The contribution of the gravitational radiation $-\sigma_{a b}^{(\ell)} \sigma^{(\ell) a b}$ and $\zeta_{a} \zeta^{a}$.

We will give more discussions on the gravitational radiation at the end of this section. For the past trapping horizon, it's also easy to get similar results like eqs.(151) and (152). Since the procedure is similar, we will not give further discussions here.

\subsubsection{Codimension-2 Surface without Einstein Condition}

Without the Einstein condition, the problem becomes complicated even in the four dimension. For an arbitrary null frame $\{\ell, n\}$ with $\ell_{a} n^{a}=-1$, the evolution 
of the energy becomes

$$
\begin{aligned}
& \mathcal{L}_{X} \mathscr{E}=\int \epsilon_{q}\left\{\left(\frac{\mathcal{E}}{n-2}\right)\left(\frac{\mathcal{L}_{X} \mathscr{A}}{\mathscr{A}}+B \theta^{(n)}\right)\right\} \\
& -\left(\frac{L}{n-2}\right) \int \epsilon_{q}\left\{\theta ^ { ( n ) } \left[A\left(\mathscr{T}_{a b} \ell^{a} \ell^{b}+\frac{1}{8 \pi G} \sigma_{a b}^{(\ell)} \sigma^{(\ell) a b}\right)+B \mathscr{T}_{a b} \ell^{a} n^{b}\right.\right. \\
& \left.\left.+\frac{1}{8 \pi G}\left(D^{c} D_{c} B-2 \omega^{c} D_{c} B-B D_{c} \omega^{c}+B \omega_{c} \omega^{c}\right)\right]\right\}
\end{aligned}
$$

Generally, the evolution vector $X^{a}=A \ell^{a}-B n^{a}$ does not satisfy the requirement that $K^{c} X_{c}=B \theta^{(n)}$ is a constant on the marginal surface. However, in the case of $B>0$, we can always rescale the null frame such that

$$
B \theta^{(n)}=-\frac{n-2}{L}
$$

is a constant on the marginal surface (Of course, $L$ is a constant on the marginal surface). It's easy to find this requirement also means $\mathcal{L}_{X} L=1$ (This selection is also used in [29, 30]). Thus, by using this special null frame, the evolution of the energy becomes

$$
\begin{aligned}
\mathcal{L}_{X} \mathscr{E}= & \int \epsilon_{q}\left\{\left(\frac{A}{B}\right)\left(\mathscr{T}_{a b} \ell^{a} \ell^{b}+\frac{1}{8 \pi G} \sigma_{a b}^{(\ell)} \sigma^{(\ell) a b}\right)\right. \\
& \left.+\mathscr{T}_{a b} \ell^{a} n^{b}+\frac{\zeta_{c} \zeta^{c}}{8 \pi G}\right\},
\end{aligned}
$$

where $\zeta_{c}$ is the same as the one in eq. (151). Considering that $X$ has to satisfy eq.(65) with $\theta^{(\ell)}=0$ and $\mathcal{L}_{X} \theta^{(\ell)}=0$, we have

$$
\mathcal{L}_{X} \mathscr{E}=\frac{1}{16 \pi G} \mathcal{L}_{X} L \int \epsilon_{q} R=\left(\frac{n-3}{n-2}\right)\left(\frac{\mathscr{E}}{\mathscr{A}}\right) \mathcal{L}_{X} \mathscr{A},
$$

where $\mathscr{E}$ takes value on the marginal surface. To get above result, we have inserted the relation $\mathcal{L}_{X} L=1$. It should be noted here: when $K^{a} X_{a}$ is a constant on the codimension- 2 surface, then we have $\mathcal{L}_{X} \mathscr{K}=0$, so eq. (116) can be transformed into eq. (117). Then, on the trapping horizon, the second term in the right hand of eq.(117) vanishes, and then we get above equation. Eq.(155) also shows the evolution of the energy inside the marginal surface can also be decomposed into two parts: The contribution of the matter and the contribution of the gravitational radiation .

When the tapping horizon is null, we have $K^{a} X_{a}=B \theta^{(n)}=0$. Eq. (153) reduces to eq.(149). So, on the null trapping horizon, the energy $\mathscr{E}$ inside the marginal surface does not evolve regardless the marginal surface is Einstein or not.

Here, some remarks are listed in order: 
(i). The deformation of the generalized Hawking mass (112), i.e., eq.(120), has a very similar form as the one in the four dimension. When the marginal surface is an Einstein manifold, the evolution of the generalized Hawking mass (112) on the trapping horizon is given by eq.(129). This result can be decomposed into the form (142). In the null frame, this result can also be expressed in eqs.(151) and (152). In the general case without the Einstein condition of the marginal surface, by choosing some special null frame, the evolution of the generalized Hawking mass can also be decomposed into the matter field part and gravitational radiation part.

(ii). The gravitational radiation is carried by $\sigma_{a b}^{(\ell)}$ (or $\sigma_{a b}^{(n)}$ in the past case). The number of the degrees of freedom of this tensor is $n(n-3) / 2$. This is just the number of graviton polarizations in $n$-dimension. In the previous discussion, we think that the term which corresponds to $\zeta_{a} \zeta^{a}$ is also a kind of gravitational radiation. However, we have to point out: the detailed physical meaning of the term $\zeta_{a} \zeta^{a}$ is still unclear. Certainly, this term really comes from the change of the gravitational field, so it's reasonable to regard it as a term of gravitational radiation.

(iii). The problem of the angular momentum: The deformation of the Hawking mass or it's generalized version in eq.(112) in some sense are not sufficient to describe the full dynamics of the trapping horizon. One has to consider the angular momentum separately [54, [55]. We can define the angular momentum to be $J_{\phi}=\int \epsilon_{q}\left(\phi^{a} \omega_{a}\right)$ for some tangent vector $\phi$ which satisfies $\mathcal{L}_{X} \phi^{a}=0$ and $D_{a} \phi^{a}=0$, then, the deformation of the angular momentum can be studied by eq. (82) (or (93)). In the four dimension, there is only one possible angular momentum. However, in the higher dimension, the situation is complicated: It's possible that there are several (not single) angular momentums associated with the trapping horizon.

(iv). Generally the surface gravity defined in eq.(140) is not a constant on the marginal surface, i.e., $D_{a} \kappa \neq 0$. In the spherically symmetric case, this kind of surface gravity is really a constant on the marginal surface. However, generally, it still evolves on the trapping horizon, i.e., $\mathcal{L}_{X} \kappa \neq 0$. This is very different from the static case in which the surface gravity is a constant on the horizon. Although we can get some energy balance like equation (for example, eq.(151)), however, frankly speaking, the definition of the surface gravity of general nonnull trapping horizon is still an open problem [49]. Of course, we hope the definition of the surface gravity can give some physically acceptable result when the system is almost equilibrium.

(v). For the null trapping horizon, the generalized Hawking mass does not evolve on the trapping horizon. Further, if null energy condition is imposed, all terms in the right hand of eq.(152) have to be vanished. So there are no dynamical version first laws associated with the null trapping horizons. To study the dynamics of the null trapping horizon, we have to consider other method, for example, the phase space version first law [13, 14, 15, 16, 17. 


\section{Horizon Dynamics without Quasilocal Energy}

Without selecting some quasilocal energy inside the codimension-2 surface, we can also study the dynamics of the trapping horizon. This kind of discussion heavily depends on the deformation equations of the expansions and the $S O(1,1)$ connection we have get in section 4. Eqs. (75) and (80) are key equations to construct some first law like equation in this formalism. Certainly, one of the most important problems in this formalism is also the definition of the surface gravity. As mentioned at the end of last section, to make the problem easy to understand, it's better to discuss some near equilibrium state at first. So, in this section, we discuss the slowly evolving trapping horizon proposed by Booth et.al. [20, 21, 22, 46. This theory just describes the trapping horizon which corresponds to the near equilibrium state. We will generalize the slowly evolving future outer trapping horizon to the past trapping horizons (sometime there are no future trapping horizons in a given spacetime). This is important to study the FLRW universe because the past trapping horizon is important in this case.

To make the discussion clear, here, we put the deformation equations of the expansions (After selecting $X$ to be the evolution vector, these are actually evolution equations), i.e., eqs.(65) and (68) into simple forms:

$$
\begin{aligned}
& \mathcal{L}_{X} \theta^{(\ell)}=-D_{c} D^{c} B+2 \omega^{c} D_{c} B-B \mathcal{L}_{n} \theta^{(\ell)}+A \mathcal{L}_{\ell} \theta^{(\ell)}, \\
& \mathcal{L}_{X} \theta^{(n)}=D_{c} D^{c} A+2 \omega^{c} D_{c} A+A \mathcal{L}_{\ell} \theta^{(n)}-B \mathcal{L}_{n} \theta^{(n)} .
\end{aligned}
$$

For the future trapping horizon, we have $\mathcal{L}_{X} \theta^{(\ell)}=0$, so the relation between $A$ and $B$ is given by a two order differential equation of $B$. Similarly, for the past trapping horizon, we have $\mathcal{L}_{X} \theta^{(n)}=0$, and the relation between $A$ and $B$ is encoded in a two order differential equation of $A$.

\subsection{Equilibrium State}

The theory of the so called slowly evolving horizon is proposed to describe the dynamics of some horizon which corresponds to the near equilibrium state. So, to study the slowly evolving horizon, firstly we have to study the horizon which corresponds to an equilibrium state. Actually, in black hole theory, the equilibrium state is described by the dynamical behavior of some horizon which is null. Of course, this means the vector $X$ is null. For the null future trapping horizon, we can assume $X_{a}=A \ell_{a}$, and then the evolution equations of the expansions become

$$
\mathcal{L}_{X} \theta^{(\ell)}=A \mathcal{L}_{\ell} \theta^{(\ell)}, \quad \mathcal{L}_{X} \theta^{(n)}=D_{c} D^{c} A+2 \omega^{c} D_{c} A+A \mathcal{L}_{\ell} \theta^{(n)} .
$$

Similarly, for the null past trapping horizon, by setting $X_{a}=-B n_{a}$, the evolution equations are simplified to be

$$
\mathcal{L}_{X} \theta^{(n)}=-B \mathcal{L}_{n} \theta^{(n)}, \quad \mathcal{L}_{X} \theta^{(\ell)}=-D_{c} D^{c} B+2 \omega^{c} D_{c} B-B \mathcal{L}_{n} \theta^{(\ell)} .
$$


Firstly, assuming the null energy condition is satisfied, then by using $\mathcal{L}_{X} \theta^{(\ell)}=$ 0 and the (cross) focusing equations (94), on the null future trapping horizon, we have

$$
\sigma_{a b}^{(\ell)}=0, \quad \mathscr{G}_{a b} \ell^{a} \ell^{b}=0,
$$

and on the null past trapping horizon, we have

$$
\sigma_{a b}^{(n)}=0, \quad \mathscr{G}_{a b} n^{a} n^{b}=0 .
$$

Above equations tell us: $K_{a b}^{(\ell)}=0$ on the null future trapping horizon, and $K_{a b}^{(n)}=0$ on the null past trapping horizon. Further, $\mathscr{G}_{a b} \ell^{a} \ell^{b}=0$ and $\mathscr{G}_{a b} n^{a} n^{b}=$ 0 just imply that there are no matter flux across the codimension- 2 surface.

Secondly, if we also require that $q_{a}{ }^{c} \mathscr{G}_{c b} \ell^{b}=0$ on the null future trapping horizon and $q_{a}{ }^{c} \mathscr{G}_{c b} n^{b}=0$ on the null past trapping horizon, then, from the Codazzi equations (33), (34) and relation (35), we get $q_{a}{ }^{e} q^{b c} \ell^{d} \mathscr{C}_{e b c d}=0$ on the null future tapping horizon and $q_{a}{ }^{e} q^{b c} n^{d} \mathscr{C}_{e b c d}=0$ on the null past trapping horizon $\left(q_{a}^{c}{ }^{c} \mathscr{G}_{c b} \ell^{b}=0\right.$ or $q_{a}^{c}{ }^{c} \mathscr{G}_{c b} \ell^{b}=0$ can be satisfied if dominant energy condition is assumed.).

Finally, from above requirements and eq.(80), it's easy to find

$$
\mathcal{L}_{X} \omega_{a}-D_{a} \kappa_{X}=0
$$

on the null trapping horizon. This result does not depend on the selection of $A$ $(B)$ in $X_{a}=A \ell_{a}\left(X_{a}=-B n_{a}\right)$. According to eq.90), it's also independent of the rescaling of the null frame $\{\ell, n\}$. Additionally, if one requires that $\omega_{a}$ does not evolve, i.e., $\mathcal{L}_{X} \omega_{a}=0$, then, from above equation or eq.(80), one gets $D_{a} \kappa_{X}=0$ on the codimension- 2 surface for both cases (future and past). This means $\kappa_{X}$ is a constant on the codimension- 2 surface. Furthermore, if $\mathcal{L}_{X} \kappa_{X}=0$ is required, then $\kappa_{X}$ is a constant on the null trapping horizon (regardless future or past).

In fact, the requirement that $\kappa_{X}$ is a constant on the null trapping horizon gives some constraints on the function $A(B)$. In the case of future, this can be found from following equations:

$$
\begin{aligned}
D_{a} \kappa_{X} & =\kappa_{\ell} D_{a} A+A D_{a} \kappa_{\ell}=0, \\
\mathcal{L}_{X} \kappa_{X} & =A\left(\kappa_{\ell} \mathcal{L}_{\ell} A+A \mathcal{L}_{\ell} \kappa_{\ell}\right)=0 .
\end{aligned}
$$

Similarly, for the past null trapping horizon, $B$ has to satisfy

$$
\begin{aligned}
D_{a} \kappa_{X} & =-\kappa_{n} D_{a} B-B D_{a} \kappa_{n}=0, \\
\mathcal{L}_{X} \kappa_{X} & =B\left(\kappa_{n} \mathcal{L}_{n} B+B \mathcal{L}_{n} \kappa_{n}\right)=0 .
\end{aligned}
$$

Since, until now, the null frame $\{\ell, n\}$ can be arbitrarily rescaled: $\{\ell, n\} \rightarrow$ $\{\lambda \ell, n / \lambda\}$, generally, $\kappa_{\ell}\left(\kappa_{n}\right)$ is not a constant on the future (past) null trapping horizon. Given a null frame $\{\ell, n\}$, we can always find some $A(B)$ to satisfy eq. (163) (164) ). Obviously, this $A(B)$ is not unique. Inversely, given an evolution vector $X^{a}$, we can always find some null frame $\{\ell, n\}$ (also not unique) 
to satisfy eqs.(163) and (164). So eqs.(163) and (164) give the relations between the foliation structure $(X)$ and the null frame.

For example, if we have select some $\lambda$ such that $\kappa_{\ell}\left(\kappa_{n}\right)$ fulfills the requirement that $\kappa_{\ell}\left(\kappa_{n}\right)$ is a constant on the future (past) null trapping horizon, then, from eq.(163) ((164) $)$, we have to set $A(B)$ such that $D_{a} A=\mathcal{L}_{\ell} A=0$ $\left(D_{a} B=\mathcal{L}_{n} B=0\right)$ on the null future (past) trapping horizon (we have assume that $\kappa_{\ell}$ and $\kappa_{n}$ are both nonvanished). Obviously, these conditions can be satisfied if we choose that $A(B)$ is a constant. In this case, it's easy to find $\kappa_{X}$ satisfies (From eqs.(25) and (42), it's easy to find $q_{a}^{c} X^{b} \nabla_{b} X_{c}=0$.)

$$
X^{a} \nabla_{a} X^{b}= \pm \kappa_{X} X^{b}
$$

where "+" corresponds to the case of future, while " -" corresponds to the case of past. This is just the usual formula to define a surface gravity. Obviously, this kind of surface gravity is defined up to a constant coefficient.

Actually, for a given function $A(B)$, we can set $\lambda$ to be proportional to $A$ with a constant coefficient (or $\lambda$ which is proportional to $1 / B$ ), and rescale the null frame such that $X^{a} \nabla_{a} X^{b}= \pm \kappa_{X} X^{b}$ (with constant $\kappa_{X}$ ) is satisfied under the resulting null frame.

It should be noted here, we have not discuss the relabeling of the foliation until now. The vector $X$ is rescaled by a factor $f(\tau)=\left(d \tau^{\prime} / d \tau\right)^{-1}$ if we relabel the foliation: $\tau \rightarrow \tau^{\prime}(\tau)$. For the null trapping horizons, this just means that $A$ or $B$ is rescaled by the factor $f$. So we can use the same procedure as before to find a special null frame such that the relation (165) is always held.

From above discussion, we find: to make that $\kappa_{X}$ is a real surface gravity given by eq. 165), it's necessary to select a special null frame to match the given foliation structure of the null trapping horizon (i.e. X). Certainly, the physics the horizon should not depend on the relabeling of the foliation and rescaling of the null frame. So different $X$ 's (with the preferred $\{\ell, n\}$ and the corresponding $\kappa_{X}$ ) in some sense are physically equivalent. In summary, the null trapping horizon can be characterized by an equivalent class which can be expressed by a triplet

$$
\left[X,\{\ell, n\}, \kappa_{X}\right] \text {. }
$$

Of course, to foliate the null trapping horizon, the simplest way is select $X^{a}=\ell^{a}$ (or $X^{a}=-n^{a}$ ). With this selection, eq.165) is automatically satisfied.

Above statement has close relation to the isolated horizon (especially in the case with constant $A(B)$ ) defined by Ashtekar el.al. [13. In those cases, one mainly focuses on the future outer trapping horizon. Here, we also study the past trapping horizon. Our discussion is independent of the selecting of the metric of the null hypersuface (degenerate). In fact, this is a rough way to reconstruct of the isolated horizon [22].

Conclusively, on these null trapping horizons, there are no gravitational radiation and matter flux, and $\kappa_{X}$ 's are constants. These properties correspond to the equilibrium state of the thermodynamics of the horizon. Further, now 
eqs. (75) and (82) just mean

$$
\left(\frac{\kappa_{X}}{2 \pi}\right) \mathcal{L}_{X} S=0, \quad \mathcal{L}_{X} J_{\phi}=0
$$

where $S \sim \int \epsilon_{q}$ and $J_{\phi} \sim \int \epsilon_{q}\left(\phi^{a} \omega_{a}\right)$ can be explained as the entropy and the angular momentum associated with the null trapping horizons. Since there are no matter flux and gravitational radiation across the null trapping horizon, these physical quantities do not change along the trapping horizons as expected. So there are no dynamical version of the first law associated with the null trapping horizons. Actually, this point have been found in the Sec.6.: the energy $\mathscr{E}$ does not evolve on the null trapping horizon. In fact, one can study the first law of the null trapping horizons by using the phase space method [13, 14, 15, 16, 17].

\subsection{Near Equilibrium State}

The near equilibrium means that $X$ is almost a null vector. This suggests that $X_{a}=A \ell_{a}-B n_{a}$ slightly deviates from a null vector. However, to characterize this small deviation, it's not sufficient to set one of $A$ or $B$ to be very small. The reason is that there are two ambiguities for the vector $X$ we have mentioned several times:

(i). There are freedoms to choose the null frame $\{\ell, n\} \rightarrow\{\lambda \ell, n / \lambda\}$ for some positive function $\lambda$, and this makes an ambiguity for $B / A$ or $A / B$ by factors $\lambda^{2}$ or $1 / \lambda^{2}$.

(ii). The relabeling of the foliation of the trapping horizon $\tau \rightarrow \tau^{\prime}(\tau)$. This makes an ambiguity that $X$ can be rescaled as $X \rightarrow X^{\prime}=f X$ with $f=\left(d \tau^{\prime} / d \tau\right)^{-1}$.

We can assume that the norm of $X$ (or $X_{a} X^{a}=2 A B$ ) approaches zero such that $X$ is almost a null vector. This will eliminate the ambiguity of rescaling of the null frame. However, since $X^{\prime}$ and $X$ are essentially equivalent to describe the trapping horizon, so the norm of the evolution vector will get a factor $|f|$ if we use $X^{\prime}$. We need some procedure to carefully treat the rescaling of the foliation.

For the null trapping horizons, we can always find some special null frame to satisfy the requirement of $X^{b} \nabla_{b} X^{a}= \pm \kappa_{X} X^{a}$ (with a constant $\kappa_{X}$ ), and above two ambiguities in some sense are fixed to get the preferred null frame and the corresponding surface gravity. However, for the general case of non-null horizon, the situation is very different. From eq.(42), it's not hard to find 3

$$
X^{b} \nabla_{b} X_{a}=\kappa_{X}\left(\epsilon_{a b} X^{b}\right)-D_{a}(A B)+\left(\mathcal{L}_{X} A\right) \ell_{a}-\left(\mathcal{L}_{X} B\right) n_{a} .
$$

Obviously, now, it's impossible to get $X^{b} \nabla_{b} X^{a}= \pm \kappa_{X} X^{a}$ by rescaling the null frame. So, principally, the strategy of the rescaling of the null frame in the null horizon case is meaningless for the non-null trapping horizon. Actually, in this case, we do not know how to select a preferred null frame to define a surface gravity associated with $X$. However, mimicking the null case, for a given

\footnotetext{
${ }^{3}$ This equation reduces to the result given in 38 when $A=1$.
} 
foliation of the trapping horizon, by rescaling the null frame, we can always set $A$ (or $B$ ) to be a constant for the future case (or the past case). Without losing generality, we can set $A=1$ ( or $B=1$ ). This procedure is similar to the null cases. Thus, for the future trapping horizon, we have

$$
X^{a}=\ell^{a}-C n^{a},
$$

while for the past trapping horizon, we have

$$
X^{a}=C \ell^{a}-n^{a} .
$$

It should be noted here, the functions $C$ 's depend on the relabeling of the foliation of the trapping horizons. Actually, the most general form of the evolution vector can be expressed as

$$
X^{a}=f(\tau) \bar{X}^{a}=f(\tau)\left(A \bar{\ell}^{a}-B \bar{n}^{a}\right) .
$$

with some null frame $\{\bar{\ell}, \bar{n}\}$. One can regard that the factor $f$ comes from the relabeling of the foliation 4 . For the future case, redefining the null frame $\bar{\ell}^{a} \rightarrow \ell^{a}=(f A) \bar{\ell}^{a}$ and $\bar{n}^{a} \rightarrow n^{a}=\bar{n}^{a} /(f A)$, we can put $X$ into the form (168) with

$$
C=f^{2} A B \text {. }
$$

Similar situation happens in the past case. By this, the relabeling of the foliation is encoded in the function $C$. So it seems that one can relabel the foliation of the trapping horizons such that $C$ 's to be arbitrary nonvanished values (Obviously, for null cases, $C$ 's are always zero and independent of any foliation structure).

Since for any kind of trapping horizon, we can always take $|C|$ 's to be arbitrarily small value by selecting the foliation parameter $\tau$, so the assumption of small $|C|$ 's is still not enough to discuss the slowly expanding behavior of the trapping horizons. To describe the almost null property, we need some quantity which is independent of the relabeling of the foliation and the rescaling of the null frame. We will discuss the cases of future and past separately.

- For the future trapping horizon, Booth et.el. [20, 21, 22, 46] give three slowly expanding conditions (here we gives a generalized $n$-dimension version):

(F-i). The so called evolving parameter $\epsilon \ll 1$ with

$$
\frac{\epsilon^{2}}{L^{2}}=\max \left[|C|\left(\left\|\sigma^{(n)}\right\|^{2}+(8 \pi G) \mathscr{T}_{a b} n^{a} n^{b}+\frac{1}{n-2} \theta^{(n)} \theta^{(n)}\right)\right]
$$

\footnotetext{
${ }^{4}$ Here, to make the discussion clear, we also require that $A$ (or $B$ ) can not be further viewed as a relabeling factor. For example, $A$ (or $B$ ) is not a constant on the codimension-2 surface, so it can not be absorbed into $f$. Certainly, in some special cases, $A$ and $B$ are both constants on the codimension-2 surface. In this case, we can absorb $A$ (or $B$ ) into the factor $f$, and the evolution vector is simply assumed to be $X^{a}=f\left(\ell^{a}-C^{\prime} n^{a}\right)\left(\right.$ or $\left.X^{a}=f\left(C^{\prime} \ell^{a}-n^{a}\right)\right)$ with some function $C^{\prime}$.
} 
(F-ii). The Ricci scalar, the $S O(1,1)$ normal connection and the energy-momentum tensor satisfy

$$
|R|, \quad\left\|\omega_{a}\right\|^{2} \quad \text { and } \quad(8 \pi G) \mathscr{T}_{a b} \ell^{a} n^{b} \preceq \frac{1}{L^{2}} ;
$$

(F-iii). The derivatives of horizon fields are at most the same order in $\epsilon$ as the (maximum of the) original fields. For example,

$$
\left\|D_{a} C\right\| \preceq \frac{C_{m}}{L}, \quad\left\|D_{a} D_{b} C\right\| \preceq \frac{C_{m}}{L^{2}} .
$$

Here, $\|\cdot\|$ is the norm of (tangent) tensor fields on the codimension-2 Riemannian manifold, while $|\cdot|$ is the absolute value of some scalar. The quantity $L$ is some length scale of the codimension- 2 surface. For example, the radius of the closed $(n-2)$ manifold: $L=\left(\mathscr{A} / \Omega_{n-2}\right)^{\frac{1}{n-2}}$ which has been defined just bellow eq.(120). $C_{m}$ is the maximum value of $|C|$ on the codimension-2 surface. The relation $E \preceq F$ means $E \leq k_{0} F$ for some constant $k_{0}$ of order one.

Before using these conditions, we give some discussions:

Firstly, $\epsilon$ defined in the condition (F-i) is independent of the relabeling of the foliation and the rescaling of the null frame. This can be easy found from the expressions of $\epsilon$ in the null frame $\{\bar{\ell}, \bar{n}\}$ bellow eq.(170):

$$
\frac{\epsilon^{2}}{L^{2}}=\max \left[\left|\frac{B}{A}\right|\left(\left\|\sigma^{(\bar{n})}\right\|^{2}+(8 \pi G) \mathscr{T}_{a b} \bar{n}^{a} \bar{n}^{b}+\frac{1}{n-2} \theta^{(\bar{n})} \theta^{(\bar{n})}\right)\right] .
$$

This is just what we hope to find: The parameter should not depend on the relabeling of the foliation and the local frame. By this consideration and the definition of $\epsilon$, the requirement of $\epsilon \ll 1$ in condition (F-i) essentially gives some constraint on the dynamical behavior of the codimension-2 surface.

Secondly, the evolution vector $X$ is not arbitrary, and it has to satisfy $\mathcal{L}_{X} \theta^{(\ell)}=0$. This gives a differential equation of $C$ :

$$
\mathcal{L}_{X} \theta^{(\ell)}=-D_{c} D^{c} C+2 \omega^{c} D_{c} C-C \mathcal{L}_{n} \theta^{(\ell)}+\mathcal{L}_{\ell} \theta^{(\ell)}=0 .
$$

This is just a special case of the first equation in (157). Furthermore, considering $D_{a} f=0$ and the expression of $\mathcal{L}_{\ell} \theta^{(\ell)}$ in eq. (94), the relation $\mathcal{L}_{X} \theta^{(\ell)}=0$ does not give any constraint on the relabeling factor $f$. This means eq.(173) is actually a equation of $A B$ in (171). Thus, the function $C / f^{2}$ is determined by the geometrical behavior (both intrinsic and extrinsic) of the codimension-2 surface. Remembering that $\epsilon \ll 1$ has some requirement on the geometry of the codimension-2 surface in the trapping horizon, so $C / f^{2}$ (or $A B$ ) is also required to satisfy some condition by the behavior of $\epsilon$. Actually, in some simple case, we can find the explicit relation between $A B$ and $\epsilon$. This situation happens in the case of the FLRW universe, and we will find it in the next section.

So the relabeling of the foliation is arbitrary until now. However, if we require

$$
|C| \preceq \epsilon^{2}
$$


then, the function $f$ has to satisfy some condition. This corresponds to some special selection of the foliation parameter $\tau$, and then we can not relabeling the foliation arbitrarily. In the following discussion, we will always assume this condition on $|C|$.

Now, let's discuss the implication of the conditions (F-i), (F-ii), (F-iii) and (174). From these slowly expanding conditions and eq.(94), it's not hard to find on the future trapping horizon, we have

$$
\left|\mathcal{L}_{n} \theta^{(\ell)}\right| \preceq \frac{1}{L^{2}}
$$

then, considering eq.(173) and $|C| \preceq \epsilon^{2}$, one gets that

$$
\left|\mathcal{L}_{\ell} \theta^{(\ell)}\right|=\left\|\sigma^{(\ell)}\right\|^{2}+8 \pi G \mathscr{T}_{a b} \ell^{a} \ell^{b} \preceq \frac{\epsilon^{2}}{L^{2}}
$$

is satisfied on the future trapping horizon. If the null energy condition is assumed, the two terms in the middle of above equation are both nonnegative. It's also easy to find

$$
K_{a b}^{(X)}=\sigma_{a b}^{(\ell)}-C K_{a b}^{(n)}=\sigma_{a b}^{(\ell)}+\mathscr{O}\left(\epsilon^{2}\right)
$$

Obviously, the first term is the order of $\epsilon$, while the terms proportional to $C$ is the order of $\epsilon^{2}$. Since $\epsilon \ll 1$, we have $K_{a b}^{(X)} \approx \sigma_{a b}^{(\ell)}$. Similarly we have $K_{a b}^{(Y)} \approx \sigma_{a b}^{(\ell)}$. With the same discussion, we get

$$
\mathscr{T}_{a b} X^{a} Y^{b}=\mathscr{T}_{a b} \ell^{a} \ell^{b}-C^{2} \mathscr{T}_{a b} n^{a} n^{b}=\mathscr{T}_{a b} \ell^{a} \ell^{b}+\mathscr{O}\left(\epsilon^{4}\right) .
$$

Here, the vector $Y$ is the dual of $X$, i.e., $Y^{a}=\ell^{a}+C n^{a}$. For other quantities, one can also get reasonable approximations.

In addition to the null energy condition, let's assume that energy -momentum tensor also satisfies dominant energy condition. This means, for every futurepointing causal vector field $Z^{a}$, the vector field $-\mathscr{T}_{a b} Z^{b}$ must be a future pointing causal vector. This assumption of energy-momentum implies

$$
g^{a c} \mathscr{T}_{a b} Z^{b} \mathscr{T}_{c d} Z^{d}=\left\|q_{a}^{b} \mathscr{T}_{b c} Z^{c}\right\|^{2}-2\left(\mathscr{T}_{a b} \ell^{a} Z^{b}\right)\left(\mathscr{T}_{c d} n^{c} Z^{d}\right) \leq 0 .
$$

By selecting $Z^{a}=\ell^{a}$, we get

$$
\left\|q_{a}^{b} \mathscr{T}_{b c} \ell^{c}\right\|^{2} \leq 2\left(\mathscr{T}_{a b} \ell^{a} \ell^{b}\right)\left(\mathscr{T}_{c d} n^{c} \ell^{d}\right) \preceq \epsilon^{2} / L^{4}
$$

and then

$$
\left\|q_{a}^{b} \mathscr{T}_{b c} \ell^{c}\right\| \preceq \frac{\epsilon}{L^{2}} .
$$

From the Codazzi equation (33) and (35) and above result, we get

$$
\left\|q_{a}^{e} q^{b c} \ell^{d} \mathscr{C}_{e b c d}\right\| \preceq \frac{\epsilon}{L^{2}} .
$$

For the equilibrium state, the function $C$ is identically vanished. So $\epsilon$ is also vanished. Obviously, above conditions of the tensors (on the future trapping 
horizon) give $\sigma_{a b}^{(\ell)}=0, \mathscr{T}_{a b} \ell^{a} \ell^{b}=0, q_{a}^{b} \mathscr{T}_{b c} \ell^{c}=0$ and $q_{a}{ }^{e} q^{b c} \ell^{d} \mathscr{C}_{e b c d}=0$. These are just the requirements for the case of the null trapping horizon.

During above discussions, all the conditions and results are focused on some given marginal surface. However, to study the evolution of this marginal surface, these conditions are still not enough. Remembering in the case of future null trapping horizon, to ensure that some physical quantities (the area and the angular momentum associated with the horizon) do not evolve, we have required the condition $\mathcal{L}_{X} \omega_{a}=0$ and $\mathcal{L}_{X} \kappa_{X}=0$. These just mean that $\omega_{a}$ and $\kappa_{X}$ do not evolve respect to the evolution vector $X^{a}$. Similarly, here there are also slowly evolving conditions :

(F-i'). $\left\|\mathcal{L}_{X} \omega_{a}\right\|$ and $\left|\mathcal{L}_{X} \kappa_{X}\right| \preceq \epsilon / L^{2} ;$

(F-ii'). $\left|\mathcal{L}_{X} \theta^{(n)}\right| \preceq \epsilon / L^{2}$.

From eq. (80) and the restriction condition of the fields (and their derivatives) on codimension-2 surface, it's easy to find

$$
\left\|D_{a} \kappa_{X}\right\| \preceq \frac{\epsilon}{L^{2}} .
$$

Considering that the absolute value of $\mathcal{L}_{X} \kappa_{X}$ also satisfies this conditions, then $\kappa_{X}$ is almost a constant on the trapping horizon (at least for some finite interval of the foliation parameter $\tau$ ). Thus, $\kappa_{X}$ can be expanded as

$$
\kappa_{X}=\kappa_{o}+\mathscr{O}(\epsilon)
$$

where $\kappa_{O}$ is the leading term of the expansion.

The condition (F-ii') just requires that $\theta^{(n)}$ also evolves slowly. It should be noted here: generally, in the definition of the future trapping horizon, there are no requirements on $\mathcal{L}_{X} \theta^{(n)}$ although that the sign of $\theta^{(n)}$ has close relation to the classification of the future trapping horizon.

- For the past trapping horizon, we can gives similar conditions to describe the slowly expanding properties:

(P-i). The evolving parameter $\epsilon \ll 1$ with

$$
\frac{\epsilon^{2}}{L^{2}}=\max \left[|C|\left(\left\|\sigma^{(\ell)}\right\|^{2}+(8 \pi G) \mathscr{T}_{a b} \ell^{a} \ell^{b}+\frac{1}{n-2} \theta^{(\ell)} \theta^{(\ell)}\right)\right] ;
$$

(P-ii). The Ricci scalar, the $S O(1,1)$ normal connection and the energy-momentum tensor satisfy

$$
|R|, \quad\left\|\omega_{a}\right\|^{2} \quad \text { and } \quad(8 \pi G) \mathscr{T}_{a b} \ell^{a} n^{b} \preceq \frac{1}{L^{2}} ;
$$

(P-iii). The derivatives of horizon fields are at most the same order in $\epsilon$ as the (maximum of the) original fields. For example,

$$
\left\|D_{a} C\right\| \preceq \frac{C_{m}}{L}, \quad\left\|D_{a} D_{b} C\right\| \preceq \frac{C_{m}}{L^{2}} .
$$


Similar to the future case, we can choose some foliation parameter such that $|C|$ is small and satisfy the condition $|C| \preceq \epsilon^{2}$.

Substituting these conditions into the expression of $\mathcal{L}_{\ell} \theta^{(n)}$ in eq. (94), it's easy to find on the past trapping horizon, we have

$$
\left|\mathcal{L}_{\ell} \theta^{(n)}\right| \preceq \frac{1}{L^{2}} .
$$

then, by setting $B=1$ and $A=C$ in eqs. (157) and considering $\mathcal{L}_{X} \theta^{(n)}=0$ on the past trapping horizon, we get

$$
\left|\mathcal{L}_{n} \theta^{(n)}\right|=\left\|\sigma^{(n)}\right\|^{2}+8 \pi G \mathscr{T}_{a b} n^{a} n^{b} \preceq \frac{\epsilon^{2}}{L^{2}} .
$$

Certainly, by assuming the null energy condition, the two terms in the middle of above equation are nonnegative separately. It's also easy to find

$$
K_{a b}^{(X)}=C K_{a b}^{(\ell)}-\sigma_{a b}^{(n)}=-\sigma_{a b}^{(n)}+\mathscr{O}\left(\epsilon^{2}\right),
$$

and

$$
K_{a b}^{(Y)}=C K_{a b}^{(\ell)}+\sigma_{a b}^{(n)}=\sigma_{a b}^{(n)}+\mathscr{O}\left(\epsilon^{2}\right) .
$$

Here, the vector $Y$ is given by $Y^{a}=C \ell^{a}+n^{a}$. Considering $\epsilon \ll 1$, we have $K_{a b}^{(X)} \approx-\sigma_{a b}^{(n)}$ and $K_{a b}^{(Y)} \approx \sigma_{a b}^{(n)}$. With the same discussion, we get

$$
\mathscr{T}_{a b} X^{a} Y^{b}=C^{2} \mathscr{T}_{a b} \ell^{a} \ell^{b}-\mathscr{T}_{a b} n^{a} n^{b}=-\mathscr{T}_{a b} n^{a} n^{b}+\mathscr{O}\left(\epsilon^{4}\right) .
$$

Since the null vector $n^{a}$ is antiself dual, i.e., $\epsilon_{a b} n^{b}=-n_{a}$, there is a sign difference from the case of the future trapping horizon. Additionally, if we assume the dominant energy condition is satisfied, then, we have

$$
\left\|q_{a}^{b} \mathscr{T}_{b c} n^{c}\right\| \preceq \frac{\epsilon}{L^{2}}, \quad\left\|q_{a}^{e} q^{b c} n^{d} \mathscr{C}_{\text {ebcd }}\right\| \preceq \frac{\epsilon}{L^{2}} .
$$

Similar to the future case, the slowly evolving conditions are given:

(P-i'). $\left\|\mathcal{L}_{X} \omega_{a}\right\|$ and $\left|\mathcal{L}_{X} \kappa_{X}\right| \preceq \epsilon / L^{2} ;$

(P-ii'). $\left|\mathcal{L}_{X} \theta^{(\ell)}\right| \preceq \epsilon / L^{2}$.

With these conditions, one can find that $\kappa_{X}$ is nearly a constant on the past trapping horizon. So it can also be expanded as $\kappa_{X}=\kappa_{o}+\mathscr{O}(\epsilon)$.

At the end of this subsection, some discussions are given as follows:

(i). In the discussion of the null trapping horizons, for some given foliation structure (some given $X$ ) of the trapping horizon, we can always get $X^{b} \nabla_{b} X^{a}= \pm \kappa_{X} X^{a}$ by selecting a special null frame. So, in some sense, we can foliate the null trapping horizon arbitrarily. However, in the non-null cases, the nonvanished functions $C$ 's carry the information of the foliation, and any restriction on them is actually a kind of restriction on the foliations. So, to discuss the slowly evolving trapping horizon, we have to select an appropriate foliation, i.e., the vector $X$. 
(ii). For some special cases in which the codimension-2 surface has enough symmetry, for example, the spherical symmetry, the problem is greatly simplified [50]. In this case, some quantities such as the shear tensor $C_{a b}{ }^{c}$ and the $S O(1,1)$ connection $\omega_{a}$ are both vanished. Further, most of the tensors are independent of the points on the codimension-2 surface.

(iii). Clausiu like equations: For the future slowly evolving trapping horizon, substituting the results (177), (178) and (183) into eq.(75), and considering slowly evolving condition (F-ii'), we get

$$
\left(\frac{\kappa_{o}}{8 \pi G}\right) \mathcal{L}_{X} \mathscr{A}=\int \epsilon_{q}\left[\mathscr{T}_{a b} \ell^{a} \ell^{b}+\sigma_{a b}^{(\ell)} \sigma^{(\ell) a b}\right],
$$

Similarly, for the past slowly evolving horizon, we have

$$
-\left(\frac{\kappa_{o}}{8 \pi G}\right) \mathcal{L}_{X} \mathscr{A}=\int \epsilon_{q}\left[\mathscr{T}_{a b} n^{a} n^{b}+\sigma_{a b}^{(n)} \sigma^{(n) a b}\right] .
$$

These are Clausius like equations. The integrals of the matter flux $\mathscr{T}_{a b} \ell^{a} \ell^{b}$ or $\mathscr{T}_{a b} n^{a} n^{b}$ and gravitational radiation $\left\|\sigma^{(\ell)}\right\|^{2}$ or $\left\|\sigma^{(n)}\right\|^{2}$ have the form of $\pm T \mathcal{L}_{X} S$. Here $S=\mathscr{A} / 4 G$ and $T=\left|\kappa_{o}\right| / 2 \pi$. Certainly, these kind of Clausius like equations hold up to the second order of the $\epsilon$. It should be noted here: On the future trapping horizon, we have $\mathcal{L}_{X} \mathscr{A}=-C \int \epsilon_{q} \theta^{(n)}$, while on the past trapping horizon, we have $\mathcal{L}_{X} \mathscr{A}=C \int \epsilon_{q} \theta^{(\ell)}$. So the sign of $\mathcal{L}_{X} \mathscr{A}$ is determined by the function $C$ and the type of the trapping horizon. Assume the null energy condition is satisfied, then, the positive temperature requires that $\delta_{X} S \geq 0$. Here, we have defined $\delta_{X}= \pm \operatorname{sign}\left(\kappa_{o}\right) \mathcal{L}_{X}$, and " + " and " - " correspond to the future and past respectively.

\section{Trapping Horizon in FLRW Universe}

In this section, as an example, we study the slowly evolving trapping horizons in the FLRW universe. We also discuss the dynamics of these kinds of trapping horizons in the formalism with the quasilocal energy (the Misner-Sharp energy) at the end of this section.

\subsection{Classification of the Trapping Horizons in FLRW Uni- verse}

Firstly, we gives the classification of the trapping horizons in the FLRW universe. The metric of the FLRW universe $(\mathcal{M}, g)$ is

$$
g=-d t^{2}+\frac{a^{2}}{1-k r^{2}} d r^{2}+a^{2} r^{2} d \Omega_{n-2}^{2},
$$

where $a=a(t)$ is scale factor and $k=0, \pm 1$, while $d \Omega_{n-2}^{2}$ is the line element of an $(n-2)$-dimensional sphere. We can decompose the metric into the form as 
eq.(11) by introducing two null vectors $\ell$ and $n$

$$
\begin{aligned}
& \ell_{a} d x^{a}=\sqrt{\frac{1}{2}}\left(-d t+\frac{a}{\sqrt{1-k r^{2}}} d r\right), \\
& n_{a} d x^{a}=\sqrt{\frac{1}{2}}\left(-d t-\frac{a}{\sqrt{1-k r^{2}}} d r\right) .
\end{aligned}
$$

So we have $h_{a b}=-\ell_{a} n_{b}-n_{a} \ell_{b}$, while $q_{a b}$ is just the metric for the sphere part, i.e.,

$$
q_{a b} d x^{a} d x^{b}=a^{2} r^{2} d \Omega_{n-2}^{2} .
$$

Obviously, $\ell$ and $n$ are both future directed. It's also easy to find $\ell_{a} \ell^{a}=$ $n_{a} n^{a}=0, \ell_{a} n^{a}=-1$ and $q_{a b} \ell^{a}=q_{a b} n^{a}=0$. Of course, there are some freedom to choose these two null vectors, for example, $\ell \rightarrow \lambda \ell$ and $n \rightarrow \lambda^{-1} n$ for some positive function $\lambda$.

From now on, we will only consider the more interesting case of the four dimension. After a simple calculation, the expansions of the sphere along these two null directions are given by

$$
\begin{aligned}
& \theta^{(\ell)}=q^{a b} \nabla_{a} \ell_{b}=\sqrt{2}\left(H+\sqrt{\frac{1}{\tilde{r}^{2}}-\frac{k}{a^{2}}}\right), \\
& \theta^{(n)}=q^{a b} \nabla_{a} n_{b}=\sqrt{2}\left(H-\sqrt{\frac{1}{\tilde{r}^{2}}-\frac{k}{a^{2}}}\right) .
\end{aligned}
$$

Here $\tilde{r}$ is defined as $\tilde{r}=a r$. It's also easy to find

$$
\begin{aligned}
& \mathcal{L}_{\ell} \theta^{(\ell)}=\dot{H}-\frac{1}{\tilde{r}^{2}}-H \sqrt{\frac{1}{\tilde{r}^{2}}-\frac{k}{a^{2}}} \\
& \mathcal{L}_{n} \theta^{(\ell)}=\dot{H}+\frac{1}{\tilde{r}^{2}}-H \sqrt{\frac{1}{\tilde{r}^{2}}-\frac{k}{a^{2}}}, \\
& \mathcal{L}_{\ell} \theta^{(n)}=\dot{H}+\frac{1}{\tilde{r}^{2}}+H \sqrt{\frac{1}{\tilde{r}^{2}}-\frac{k}{a^{2}}}, \\
& \mathcal{L}_{n} \theta^{(n)}=\dot{H}-\frac{1}{\tilde{r}^{2}}+H \sqrt{\frac{1}{\tilde{r}^{2}}-\frac{k}{a^{2}}} .
\end{aligned}
$$

From the expansions in eqs.(195) and (196), it's easy to find that: when $H<0$, we always have $\theta^{(n)}<0$. So the trapping horizon is given by $\theta^{(\ell)}=0$, and this implies relations

$$
\sqrt{\frac{1}{\tilde{r}^{2}}-\frac{k}{a^{2}}}=-H, \quad \frac{1}{\tilde{r}^{2}}=H^{2}+\frac{k}{a^{2}} .
$$

After substituting above relations, eq.(197) becomes

$$
\begin{aligned}
\mathcal{L}_{\ell} \theta^{(\ell)} & =\dot{H}-\frac{k}{a^{2}}, & \mathcal{L}_{n} \theta^{(\ell)} & =\dot{H}+2 H^{2}+\frac{k}{a^{2}}, \\
\mathcal{L}_{\ell} \theta^{(n)} & =\dot{H}+\frac{k}{a^{2}}, & \mathcal{L}_{n} \theta^{(n)} & =\dot{H}-2 H^{2}-\frac{k}{a^{2}} .
\end{aligned}
$$


These mean that the marginal surface is always future. Further, the outer or inner of the marginal surface is determined by the sign of $\dot{H}+2 H^{2}+k / a^{2}$. So the marginal surface is outer if $\dot{H}-k / a^{2}<-2\left(H^{2}+k / a^{2}\right)$ and inner if $\dot{H}-k / a^{2}>-2\left(H^{2}+k / a^{2}\right)$. When the null energy condition is satisfied, we always have $\dot{H}-k / a^{2} \leq 0$. So the marginal surface may be inner or outer.

For $H>0$, we always have $\theta^{(\ell)}>0$. When $\theta^{(n)}=0$, we have

$$
\sqrt{\frac{1}{\tilde{r}^{2}}-\frac{k}{a^{2}}}=H, \quad \frac{1}{\tilde{r}^{2}}=H^{2}+\frac{k}{a^{2}}
$$

and now eqs.(197) becomes

$$
\begin{array}{ll}
\mathcal{L}_{\ell} \theta^{(\ell)}=\dot{H}-2 H^{2}-\frac{k}{a^{2}}, & \mathcal{L}_{n} \theta^{(\ell)}=\dot{H}+\frac{k}{a^{2}}, \\
\mathcal{L}_{\ell} \theta^{(n)}=\dot{H}+2 H^{2}+\frac{k}{a^{2}}, & \mathcal{L}_{n} \theta^{(n)}=\dot{H}-\frac{k}{a^{2}} .
\end{array}
$$

So the marginal surface is always past. The marginal surface is outer if $\dot{H}-$ $k / a^{2}>-2\left(H^{2}+k / a^{2}\right)$ and inner if $\dot{H}-k / a^{2}<-2\left(H^{2}+k / a^{2}\right)$. When the null energy condition is satisfied, we always have $\dot{H}-k / a^{2} \leq 0$. So there are some ranges in which the marginal surface is outer or inner if we do not impose some additional energy conditions.

It's easy to find, in the cases with $H=0$, we always have $\theta^{(\ell)}>0$ and $\theta^{(n)}<0$, so there are no trapping horizons in this case. This is expectable because now the spacetime is actually a flat spacetime.

The FLRW universe is a typical spherically symmetric spacetime, so, as discussed before, the components of the evolution vector $X$, i.e., $A$ and $B$, are functions which only depend on the coordinates $t$ and $r$. So they are constants on the marginal surface. It's easy to find, for $H<0$, on the future trapping horizon, the relation $\mathcal{L}_{X} \theta^{(\ell)}=0$ gives

$$
A \mathcal{L}_{\ell} \theta^{(\ell)}=B \mathcal{L}_{n} \theta^{(\ell)} .
$$

With the null energy condition, from eq.(94), we always have $\mathcal{L}_{\ell} \theta^{(\ell)} \leq 0$. Thus, for the outer marginal surface, we have $\mathcal{L}_{n} \theta^{(\ell)}<0$, then, $A$ and $B$ have same signs. While for the inner marginal surface, we have $\mathcal{L}_{n} \theta^{(\ell)}>0, A$ and $B$ have opposite signs. In both cases, the relation between $A$ and $B$ is given by

$$
B=A\left(\frac{\mathcal{L}_{\ell} \theta^{(\ell)}}{\mathcal{L}_{n} \theta^{(\ell)}}\right)=A\left(\frac{\dot{H}-k / a^{2}}{\dot{H}+2 H^{2}+k / a^{2}}\right) .
$$

In the cases $H>0$, we have past trapping horizon. It's easy to find the relation $\mathcal{L}_{X} \theta_{(n)}=0$ on the trapping horizon gives

$$
A \mathcal{L}_{\ell} \theta^{(n)}=B \mathcal{L}_{n} \theta^{(n)} .
$$

By using eq.(94), with the null energy condition, we always have $\mathcal{L}_{n} \theta^{(n)} \leq 0$. So, for the outer marginal surface, we have $\mathcal{L}_{\ell} \theta^{(n)}>0$, and then $A$ and $B$ have 
opposite signs. While for the inner marginal surface, we get $\mathcal{L}_{\ell} \theta^{(n)}<0$, so $A$ and $B$ have same signs. In both cases, $A$ is given by

$$
A=B\left(\frac{\mathcal{L}_{n} \theta^{(n)}}{\mathcal{L}_{\ell} \theta^{(n)}}\right)=B\left(\frac{\dot{H}-k / a^{2}}{\dot{H}+2 H^{2}+k / a^{2}}\right) .
$$

Conclusively, in FLRW universe, for the future trapping horizon, we can set $X_{a}=A\left(\ell_{a}-\alpha n_{a}\right)$ with $\alpha$ given by

$$
\alpha=\frac{\dot{H}-k / a^{2}}{\dot{H}+2 H^{2}+k / a^{2}} .
$$

For the past trapping horizon, $X$ has form $X_{a}=B\left(\alpha \ell_{a}-n_{a}\right)$ with the same $\alpha$ given in above equation. The classification of "outer" and "inner" are indicated by the sign of $\alpha$.

The future trapping horizon is outer when $\alpha>0$, and inner when $\alpha<0$. Since in the future cases, we have $X^{a} X_{a}=2 A^{2} \alpha$, so the future outer trapping horizon is a spacelike hypersurface, while the future inner trapping horizon is a timelike hypersurface.

Similarly, the past trapping horizon is outer when $\alpha<0$ and inner when $\alpha>0$. In this case, we have $X^{a} X_{a}=2 B^{2} \alpha$, so the past outer trapping horizon is timelike, while the past inner trapping horizon is spacelike.

We have to mention some special cases: For the future trapping horizon, if $\mathcal{L}_{\ell} \theta^{(\ell)}=0$, then from eq.(202), we have $\alpha=0$. The trapping horizon reduces to a null hypersurface. Similarly, the past trapping horizon also reduces to a null hypersurface when $\alpha=0$.

So, for the future and the past trapping horizons, the general form of the vector $X^{a}$ is given by $A\left(\ell^{a}-\alpha n^{a}\right)$ and $B\left(\alpha \ell^{a}-n^{a}\right)$ respectively. Certainly, different $A$ 's and $B$ 's correspond to the different foliations of the trapping horizons. However, it should be noted here: $A$ and $B$ are both constants on the codimension-2 surface. So, for the future trapping horizon, we can view the factor $A$ as a factor $f$ provided by some relabeling of the foliation. Similarly, for the past trapping horizon, $B$ can be viewed as a relabeling factor by selecting some foliation parameter $\tau$. By this consideration, the evolution vector $X^{a}$ has the form

$$
X^{a}=f\left(\ell^{a}-\alpha n^{a}\right)
$$

in the future case, and

$$
X^{a}=f\left(\alpha \ell^{a}-n^{a}\right)
$$

in the past case. The null vector still has the form (193) and (194). In the following discussion, we will fix $f$ to be unit. We will find this selection of $f$ consists with the requirement $|C|=\left|f^{2} \alpha\right| \preceq \epsilon^{2}$. Here, $\alpha$ plays the role of $A B$ in eq.(171). 


\subsection{Null Trapping Horizons in FLRW Universe}

For the null trapping horizon, the function $\alpha$ is identically vanished. From the expression of $\alpha$ in (206), we have

$$
\dot{H}-k / a^{2}=0
$$

on the trapping horizons. The surface gravity associated with $\ell^{a}$ for the null future trapping horizon is given by

$$
\kappa_{\ell}=\frac{H}{\sqrt{2}} .
$$

The condition that $\kappa_{X}$ (now $X^{a}=\ell^{a}$ ) is a constant on the null future trapping horizon gives $\mathcal{L}_{\ell} \kappa_{\ell}=\dot{H} / 2=0$ (So we can only consider the case with $k=0$ ). This means $H$ is a negative constant on the horizon. It's also easy to find

$$
\theta^{(n)}=2 \sqrt{2} H, \quad \mathcal{L}_{n} \theta^{(\ell)}=2 H^{2}, \quad \mathcal{L}_{\ell} \theta^{(n)}=0, \quad \mathcal{L}_{n} \theta^{(n)}=-2 H^{2} .
$$

So there are only null future inner trapping horizon.

Similarly, for the past case, the surface gravity is given by

$$
\kappa_{n}=-\frac{H}{\sqrt{2}} .
$$

and $\mathcal{L}_{n} \kappa_{n}=0$ also gives that $\dot{H}=0$. So $H$ is a positive constant on the past null trapping horizon. We also have

$$
\theta^{(\ell)}=2 \sqrt{2} H, \quad \mathcal{L}_{\ell} \theta^{(n)}=2 H^{2}, \quad \mathcal{L}_{n} \theta^{(\ell)}=0, \quad \mathcal{L}_{\ell} \theta^{(\ell)}=-2 H^{2}
$$

This means there are only null past outer trapping horizon.

Conclusively, on the null trapping horizons (future and past), the Hubble parameter $H$ is always a constant. These kinds of horizons exist only when $k=0$. Further, only inner horizon exists in the future case, and only outer horizon exists in the past case. In the following discussions, we always set $k=0$. It should be noted here: the surface gravity (209) (or (211)) is defined up to a positive constant coefficient.

\subsection{Slowly Evolving Trapping Horizon in FLRW Universe}

Since the spherical symmetry, it's very simple to study the slowly evolving properties of the trapping horizon in the FLRW unverse. In this case, most of the scalars on the codimension-2 surface are constants. For example, from the definition, the evolving parameter $\epsilon$ in the condition $(\mathrm{F}-\mathrm{i}$ ) becomes (we only consider the four dimension case, and choose $L$ to be the radius $\tilde{r}=1 /|H|$ for $k=0$.)

$$
\frac{\epsilon^{2}}{\tilde{r}^{2}}=|\alpha|\left(\mathscr{G}_{a b} n^{a} n^{b}+\frac{1}{2} \theta^{(n)} \theta^{(n)}\right) .
$$


Similarly, for the past trapping horizon, the condition $(\mathrm{P}-\mathrm{i})$ is given by

$$
\frac{\epsilon^{2}}{\tilde{r}^{2}}=|\alpha|\left(\mathscr{G}_{a b} \ell^{a} \ell^{b}+\frac{1}{2} \theta^{(\ell)} \theta^{(\ell)}\right) .
$$

Since $f$ is chosen to be unit, so we have $|C|=|\alpha|$. Straightforward calculation shows: in both cases, on the trapping horizons, $\epsilon$ 's are given by

$$
\epsilon^{2}=|\alpha|\left(4-\frac{\dot{H}}{H^{2}}\right) .
$$

Remembering that we are considering the trapping horizons which are near the null future inner trapping horizon or the null past outer trapping horizon, so the functions $\alpha$ 's are assumed to be negative. By defining

$$
s=-\frac{\dot{H}}{H^{2}}>0,
$$

then, from the expression of $\alpha$ in eq.(206), we have

$$
\alpha=-\frac{s}{2-s} .
$$

To ensure that $\alpha<0$, we have to require $s<2$. The evolution parameter $\epsilon$ now has a simple form

$$
\epsilon^{2}=s\left(\frac{4+s}{2-s}\right) .
$$

It should be emphasized: the result of $\epsilon$ in above equation is independent of the rescaling of the null frame and the relabeling of the foliation. Under the relabeling of the foliation, we have $\alpha \rightarrow f^{2} \alpha$, while the term inside the round brackets in eq.(213) (or (214)) will accept a factor $1 / f^{2}$. So $\epsilon$ is invariant.

By eq.(218), we can express $s$ as a function of $\epsilon$. Considering $\epsilon \ll 1$, we have

$$
s=-\frac{\dot{H}}{H^{2}} \approx \frac{\epsilon^{2}}{2} \ll 1 .
$$

So eq.(217) gives $|\alpha| \approx \epsilon^{2} / 4$. Actually, from eqs.(218) and (217), we can express $\alpha$ as a function of $\epsilon$ explicitly. On the other hand, if we do not choose $f=1$, then we have $|C|=\left|f^{2} \alpha\right| \approx f^{2} \epsilon^{2} / 4$. To ensure the relation (174), $f^{2}$ has to be finite and order one. Certainly, the selection of $f=1$ satisfies this requirement.

It's easy to find that

$$
\mathcal{G}_{a b} \ell^{a} n^{b}=\frac{3}{\tilde{r}^{2}}+\dot{H}=\frac{3-s}{\tilde{r}^{2}} .
$$

So, when $s \ll 1$, the conditions (F-ii) and (P-ii) are easily satisfied, i.e., $\mathcal{G}_{a b} \ell^{a} n^{b} \preceq$ $1 / \tilde{r}^{2}$. Obviously, the conditions (F-iii) and (P-iii) are trivially satisfied. Thanks to the spherical symmetry and the Weyl flat of the FLRW universe, eqs.(180), 
(181) and (189) are also trivially satisfied even without any energy condition (In the general case, the dominant energy condition is required).

For the future inner trapping horizon $(H<0)$, the slowly evolving conditions (F-i') and (F-ii') reduce to $\left|\mathcal{L}_{X} \kappa_{X}\right| \preceq \epsilon / \tilde{r}^{2}$ and $\left|\mathcal{L}_{X} \theta^{(n)}\right| \preceq \epsilon / \tilde{r}^{2}$. It's easy to find

$$
\mathcal{L}_{X} \theta^{(n)}=-H^{2} s\left(1+\frac{2+s}{2-s}\right) .
$$

Therefore, from eq.(219), we have

$$
\left|\mathcal{L}_{X} \theta^{(n)}\right| \preceq \epsilon^{2} H^{2}=\epsilon^{2} / \tilde{r}^{2} .
$$

So, when $\epsilon \ll 1,\left|\mathcal{L}_{X} \theta^{(n)}\right| \preceq \epsilon / \tilde{r}^{2}$ is automatically satisfied. After substituting $\kappa_{\ell}=H / \sqrt{2}$ and $\kappa_{n}=-H / \sqrt{2}$, we find

$$
\kappa_{X}=\frac{H}{\sqrt{2}}\left(1-\frac{s}{2-s}\right)<0 .
$$

After a simple calculation, we have

$$
\mathcal{L}_{X} \kappa_{X}=-\frac{2 H^{2} s}{(2-s)^{3}}\left[2-s+s^{2}+\left(\frac{\ddot{H}}{\dot{H} H}\right)\right] .
$$

Thus, if we require

$$
\left|\frac{\ddot{H}}{H^{3}}\right| \preceq \epsilon,
$$

then we have $\left|\mathcal{L}_{X} \kappa_{X}\right| \preceq \epsilon / \tilde{r}^{2}=\epsilon H^{2}$. The condition (223) means $\dot{H}$ is also required to be slowly evolving.

In the past outer case $(H>0)$, the conditions $\left(\mathrm{P}-\mathrm{i}^{\prime}\right)$ and (P-ii') reduce to $\left|\mathcal{L}_{X} \kappa_{X}\right| \preceq \epsilon / \tilde{r}^{2}$ and $\left|\mathcal{L}_{X} \theta^{(\ell)}\right| \preceq \epsilon / \tilde{r}^{2}$. A simple calculation shows: on the past outer trapping horizon, we have

$$
\mathcal{L}_{X} \theta^{(\ell)}=H^{2} s\left(1+\frac{2+s}{2-s}\right) .
$$

So the condition $\left|\mathcal{L}_{X} \theta^{(\ell)}\right| \preceq \epsilon / \tilde{r}^{2}$ is also automatically satisfied when $\epsilon \ll 1$. Now, it's easy to find the surface gravity $\kappa_{X}$ is given by

$$
\kappa_{X}=\frac{H}{\sqrt{2}}\left(1-\frac{s}{2-s}\right)>0
$$

and

$$
\mathcal{L}_{X} \kappa_{X}=\frac{2 H^{2} s}{(2-s)^{3}}\left[2-s+s^{2}+\left(\frac{\ddot{H}}{\dot{H} H}\right)\right] .
$$

So the condition on $\left|\mathcal{L}_{X} \kappa_{X}\right|$ gives same constraint as the one of the future inner trapping horizon, i.e. $|\ddot{H}| \preceq \epsilon H^{3}$. 
Conclusively, the requirement of the evolving parameter $\epsilon \ll 1$ automatically implies that $s=-\dot{H} / H^{2}$ is very small. While the slowly evolving condition of $\kappa_{X}$ requires that $\left|\ddot{H} / H^{3}\right|$ is also a small quantity.

For the slowly evolving past trapping horizon $(H>0), s=-\dot{H} / H^{2}$ is very small. This is just one of the condition of the slow-roll inflation. Additionally, the slowly evolving condition also requires that $\left|\ddot{H} / H^{3}\right|$ is small to ensure that the surface gravity $\kappa_{X}$ changes slowly on the trapping horizon. This way, the system is near an equilibrium state. This requirement may has some relation to the second condition of the slow-roll inflation scenario. Of course, here, we have not introduce any scalar field and the corresponding potential.

For the past horizon, from eq.(191), we have

$$
-\frac{\kappa_{o}}{8 \pi G} \mathcal{L}_{X} \mathscr{A}=\int \epsilon_{q} \mathscr{T}_{a b} n^{a} n^{b} .
$$

Up to second order of $\epsilon$ (or the first order of $s$ ). Actually, it's easy to find

$$
\mathcal{L}_{X} \mathscr{A}=-\sqrt{2}\left(\frac{8 \pi}{H}\right)\left(\frac{s}{2-s}\right) \approx-\sqrt{2}\left(\frac{4 \pi}{H}\right) s+\mathscr{O}\left(s^{2}\right)
$$

So we have $\mathcal{L}_{X} \mathscr{A}<0$. This just means that $\mathscr{A}$ decreases along $X$ direction. Noted that $X^{a}=-n^{a}$ if $s=0$, so $X$ is past pointing. By this consideration, the negative $\mathcal{L}_{X} \mathscr{A}$ just means the area of the marginal sphere of the past trapping horizon increases along the future direction. The leading order of $\kappa_{X}$ is $H / \sqrt{2}$, so we have $-\left(\kappa_{o} / 8 \pi G\right) \mathcal{L}_{X} \mathscr{A}=s / 2 G+\mathscr{O}\left(s^{2}\right)$. It's also easy to find

$$
\int \epsilon_{q} \mathscr{T}_{a b} n^{a} n^{b}=\frac{s}{2 G} .
$$

Thus, eq. (191) holds up to the second order of $\epsilon$. It should be emphasized here: the surface gravity $\kappa_{X}$ is defined up to a constant factor. Actually, the factor $\sqrt{2}$ in $\kappa_{X}$ comes from the selection of the null vectors (193) and (194). From this Clausius relation like equation, we have

$$
T=\frac{\kappa_{X}}{2 \pi} \sim \frac{H}{2 \pi}\left(1-\frac{s}{2}\right)+\mathscr{O}\left(\epsilon^{4}\right) .
$$

\subsection{Quasilocal Energy and Horizon Dynamics}

To compare with the results for the slowly evolving trapping horizon in the FLRW universe, in this subsection, we also consider the case where $k=0$. The quasilocal energy (106) (or the energy (112)) inside the sphere with the radius $\tilde{r}$ is

$$
\mathscr{E}=\frac{\tilde{r}}{2 G}\left(1-\nabla_{a} \tilde{r} \nabla^{a} \tilde{r}\right) .
$$

It's easy to find the effective surface gravity $\bar{\kappa}$ in eq. (125) is given by $\bar{\kappa}=1 / 2 \tilde{r}=$ $|H| / 2$ which has the same form of the surface gravity of Schwarzschild black hole. While the surface gravity $\kappa$ in eq.(127) becomes

$$
\frac{\kappa}{2 \pi}=-\frac{|H|}{2 \pi}\left(1-\frac{s}{2}\right)
$$


where $s$ is defined in eq.(216). So the temperature of the past outer trapping horizon is $T=|\kappa| / 2 \pi=H(1-s / 2) / 2 \pi$.

If we omit the factors $\sqrt{2}$ in eqs.(221) and (225), the temperatures corresponding to $\kappa$ and $\kappa_{X}$ are coincided up to the second order of $\epsilon$. In the description with the quasilocal energy, the Clausius relation is given by (corresponding to eq.(227)

$$
A \psi_{a} X^{a}=\frac{\kappa}{2 \pi} \mathcal{L}_{X} S
$$

where $S=\mathscr{A} / 4 G$ and $X$ is the evolution vector of the trapping horizon. Of course, above equation is exactly held on the trapping horizon. More detailed discussions of the thermodynamics of the FLRW universe in various gravity theories can be found in [56, 57, 58, 59, 60, 62, 63, 64, 65, and related topics can be found in a review paper 66.

\section{Conclusion and Discussion}

In this paper, we have studied the deformation of some spacelike submanifold with an arbitrary codimension. By requiring the projection operator is Lie dragging along a given normal vector $X$, we get eq.(50). In the case of codimension-1, it reduces to the evolution equation of the extrinsic curvature of the spacelike hypersurface (56) (and eq.(55)). Even in this simple case, there are also some interest applications on thermodynamics. For example, in static case, the evolution equation (55) becomes

$$
N \mathscr{R}_{a b} u^{a} u^{b}=D^{a} D_{a} N .
$$

By defining the so called Tolman-Komar mass $M_{K}=\int \epsilon_{S} N \mathscr{R}_{a b} u^{a} u^{b}$ inside some $(n-2)$-closed surface $S$ embedded in the hypersurface, one can give some discussion on the thermodynamics associated with the surface $S$. More details can be found in 67, 68, 69. In the more interesting case of codimension-2, eq.(50) reduces to eq.(60), i.e.,

$$
\begin{aligned}
\mathcal{L}_{X} \theta^{(Y)}= & -\left(\mathscr{G}_{a b}+K_{c d a} K^{c d}{ }_{b}\right)\left[X^{a} Y^{b}-h^{a b}\left(X_{e} Y^{e}\right)\right] \\
& +\frac{1}{2}\left(R-K_{a b c} K^{a b c}-K_{c} K^{c}\right) \cdot\left(X_{e} Y^{e}\right) \\
& -Y^{e} \tilde{D}_{c} \tilde{D}^{c} X_{e}-K_{c}\left(X^{e} \nabla_{e} Y^{c}\right) .
\end{aligned}
$$

This result is frame independent, and it reduces to the well known (cross) focusing equations after selecting a local null frame. The deformation of the $S O(1,1)$ connection is given by

$$
\mathcal{L}_{X} \omega_{a}-D_{a} \kappa_{X}=\left(\frac{n-3}{n-2}\right) D_{a} \theta^{(Y)}-D_{c} \sigma_{a}^{(Y) c}+K_{c} \tilde{D}_{a} Y^{c}+q_{a}^{b} Y^{c} \mathscr{G}_{b c}
$$

with $Y_{a}=\epsilon_{a b} X^{b}$ if we define an appropriate "surface gravity" $\kappa_{X}$. It's just a Damour-Navier-Stokes like equation if $X$ is self-dual or anti-self-dual. We 
have investigated the relation between these deformation equations and the dynamics of the trapping horizon in two different formalisms: with and without introducing some quasilocal energy. In the first formalism, we have proposed a generalized energy (112) in the higher dimension Einstein gravity theory, i.e.,

$$
\mathscr{E}=\frac{\left(\int \epsilon_{q}\right)^{\frac{n-3}{n-2}}}{16 \pi G\left(\Omega_{n-2}\right)^{\frac{1}{n-2}}(n-3)}\left\{\frac{\int \epsilon_{q} R}{\left(\int \epsilon_{q}\right)^{\frac{n-4}{n-2}}}-\left(\frac{n-3}{n-2}\right) \frac{\int \epsilon_{q} K_{c} K^{c}}{\left(\int \epsilon_{q}\right)^{\frac{n-4}{n-2}}}\right\} .
$$

This energy reduces to the Hawking energy in the four dimension. In the case of general spherical symmetry, it reduces to the Misner-Sharp energy in the higher dimension. We have also studied the deformation of this quasilocal energy, and the deformation equation has been given in eq. (120). Once the requirement $\mathcal{L}_{X} \mathscr{K}=0$ is fulfilled, on the trapping horizon, we always have

$$
\mathcal{L}_{X} \mathscr{E}=\left(\frac{n-3}{n-2}\right)\left(\frac{\mathscr{E}}{\mathscr{A}}\right) \mathcal{L}_{X} \mathscr{A}
$$

This result is the total variation (evolution) of the energy on the trapping horizon, and it can be decomposed into two parts as in the four dimension: contributions from the matter fields $\left(\mathscr{T}_{a b}\right)$ and the contribution from gravitational radiation $\left(\|\sigma\|^{2}\right.$ and $\left.\|\zeta\|^{2}\right)$. When the marginal surface is Einstein, we also study the first law like equation of the trapping horizon. It has a similar form as the one with the spherical symmetry. However, generally, it's impossible to define a surface gravity which is a constant on the marginal surface. Further, it also evolves on the trapping horizon even in the spherically symmetric case. This means the system is generally nonequilibrium (even far from equilibrium point) if we regard the temperature is proportional to the surface gravity. To make the problem clear, we have studied some near equilibrium state by considering the slowly evolving trapping horizon proposed by Booth in the formalism without the quasilocal energy.

To study the past trapping horizon in the FLRW universe, we generalize the definition of the slowly evolving future outer trapping horizon to the past trapping horizon. We find, for the slowly evolving past trapping horizon, the Clausius like equation is modified to be

$$
-\left(\frac{\kappa_{o}}{8 \pi G}\right) \mathcal{L}_{X} \mathscr{A}=\int \epsilon_{q}\left[\mathscr{T}_{a b} n^{a} n^{b}+\left\|\sigma^{(n)}\right\|^{2}\right] .
$$

After classifying the trapping horizon in the FLRW universe, as an example, we study the slowly evolving trapping horizon in this spacetime. We find, to require the past trapping horizon in the FLRW universe to be slowly evolving, the Hubble parameter has to satisfy $-\dot{H} / H^{2} \approx \epsilon^{2} / 2 \ll 1$. Further, $|\ddot{H}| / H^{3}$ is required to be (at most) the order of $\epsilon$. These conditions have close relation to the scenario of the slow-roll inflation. We also compare the temperature defined in the formalism with the quasilocal energy $(T=|\kappa / 2 \pi|)$ and the temperature defined in the formalism without the quasilocal energy $\left(T=\left|\kappa_{X} / 2 \pi\right|\right)$. We find these two temperatures are essentially the same up to the second order of the slowly evolution parameter $\epsilon$. 


\section{Acknowledgement}

The author would like to thank Prof. Rong-Gen Cai and Prof. Nobuyoshi Ohta for their long term encouragement and kind help. The author would like to thank Prof. K.i.Maeda, Prof. S.Mukohyama and Prof. T.Shiromizu for their useful discussions on the surface gravity and the generalized energy form. The author also thanks Dr.Masato Minamitsuji, Dr.Masato Nozawa and Dr.ChulMoon Yoo for their useful discussions and kind help. The author would like to thank Dr.Booth for his valuable suggestions and comments. This work is supported by JSPS fellowship No.P09225.

\section{Appendix A}

In this appendix, we give a detailed derivation of eq.(50). It's easy to find the first term in eq.(49) is given by

$$
\begin{aligned}
& q_{a}^{c} q_{b}{ }^{d} X^{e} \nabla_{e} K_{c d}^{(Y)}=q_{a}^{c} q_{b}{ }^{d} X^{e} \nabla_{e}\left(q_{c}{ }^{f} q_{d}{ }^{g} \nabla_{f} Y_{g}\right) \\
& =q_{a}{ }^{c} q_{b}{ }^{g} X^{e} \nabla_{e} q_{c}{ }^{f} \nabla_{f} Y_{g}+q_{a}^{f} q_{b}{ }^{d} X^{e} \nabla_{e} q_{d}^{g} \nabla_{f} Y_{g}+q_{a}^{f} q_{b}{ }^{g} X^{e} \nabla_{e} \nabla_{f} Y_{g} \\
& =\left(q_{b}{ }^{g} \nabla_{f} Y_{g}\right) \tilde{D}_{a} X^{f}+\left(q_{a}{ }^{f} \nabla_{f} Y_{g}\right) \tilde{D}_{b} X^{g}+q_{a}^{f} q_{b}{ }^{g} X^{e} \nabla_{e} \nabla_{f} Y_{g} \\
& =\left(q_{b}{ }^{g} \nabla_{f} Y_{g}\right)\left(\tilde{D}_{a} X^{f}\right)+\left(\tilde{D}_{a} Y_{g}\right)\left(\tilde{D}_{b} X^{g}\right) \\
& \quad+q_{a}{ }^{f} q_{b}{ }^{g} X^{e} Y^{h} \mathscr{R}_{e f g h}+\underline{q_{a}{ }^{f} q_{b}{ }^{g} X^{e} \nabla_{f} \nabla_{e} Y_{g}} .
\end{aligned}
$$

Here, from second line to third line, we have used eq.(41). Again, by using eq.(41) or eq.(42), we have

$$
\begin{aligned}
& q_{a}{ }^{f} q_{b}{ }^{g} X^{e} \nabla_{f} \nabla_{e} Y_{g}=-q_{a}{ }^{f} \nabla_{f}\left(Y_{c} \tilde{D}_{b} X^{c}\right) \\
& -q_{a}{ }^{f} q_{b}{ }^{g} \nabla_{f} X^{e} \nabla_{e} Y_{g}-q_{a}{ }^{f} \nabla_{f} q_{b}{ }^{g} X^{e} \nabla_{e} Y_{g} \\
& =-\left(\tilde{D}_{a} Y_{c}\right)\left(\tilde{D}_{b} X^{c}\right)-\underline{q_{a}{ }^{f} Y_{c} \nabla_{f}\left(\tilde{D}_{b} X^{c}\right)} \\
& -K^{(X) c} K_{c b}^{(Y)}-\left(q_{b}{ }^{g} \nabla_{f} Y_{g}\right)\left(\tilde{D}_{a} X^{f}\right)-q_{a}{ }^{f} \nabla_{f} q_{b}{ }^{g} X^{e} \nabla_{e} Y_{g} .
\end{aligned}
$$

It's also easy to find

$$
\begin{aligned}
& q_{a}^{f} Y_{c} \nabla_{f}\left(\tilde{D}_{b} X^{c}\right)=K_{a}^{(Y) c} K_{c b}^{(X)}+q_{a}^{f} q_{b}^{e} Y^{g} \nabla_{f} \nabla_{e} X_{g} \\
& \quad+q_{a}^{f} \nabla_{f} q_{b}{ }^{g} Y^{e} \nabla_{g} X_{e} .
\end{aligned}
$$

So we get

$$
\begin{aligned}
q_{a}{ }^{f} q_{b}{ }^{g} X^{e} \nabla_{f} \nabla_{e} Y_{g}=-q_{a}^{f} \nabla_{f}\left(Y_{c} \tilde{D}_{b} X^{c}\right) \\
\quad-q_{a}{ }^{f} q_{b}{ }^{g} \nabla_{f} X^{e} \nabla_{e} Y_{g}-q_{a}{ }^{f} \nabla_{f} q_{b}{ }^{g} X^{e} \nabla_{e} Y_{g} \\
=-\left(\tilde{D}_{a} Y_{c}\right)\left(\tilde{D}_{b} X^{c}\right)-K^{(Y) c}{ }_{a}{ }^{(X)} K_{c b}^{(X)}-K^{(X) c} K_{c b}^{(Y)} \\
\quad-\left(q_{b}{ }^{g} \nabla_{f} Y_{g}\right)\left(\tilde{D}_{a} X^{f}\right)-q_{a}{ }^{f} \nabla_{f} q_{b}{ }^{g} Y^{e} \nabla_{g} X_{e} \\
\quad-q_{a}{ }^{f} \nabla_{f} q_{b}{ }^{g} X^{e} \nabla_{e} Y_{g}-q_{a}^{f} q_{b}{ }^{e} Y^{g} \nabla_{f} \nabla_{e} X_{g} .
\end{aligned}
$$


Then, after substituting above equation into eq.(233), we find

$$
\begin{aligned}
& q_{a}{ }^{c} q_{b}{ }^{d} X^{e} \nabla_{e} K_{c d}^{(Y)}=q_{a}{ }^{f} q_{b}{ }^{g} X^{e} Y^{h} \mathscr{R}_{e f g h}-q_{a}{ }^{f}{ }_{b}{ }^{e} Y^{g} \nabla_{f} \nabla_{e} X_{g}, \\
& \quad-K^{(X) c} K_{c b}^{(Y)}-K_{b}^{(X)^{(X}} K_{c a}^{(Y)}+K_{a b}^{(Z)} .
\end{aligned}
$$

where $Z_{a}=\mathcal{L}_{X} Y_{a}$. Substituting above equations into eq.(49), we arrive at

$$
\mathcal{L}_{X} K_{a b}^{(Y)}=K_{a b}^{(Z)}+q_{a}{ }^{f} q_{b}{ }^{g} X^{e} Y^{h} \mathscr{R}_{e f g h}-\underline{q_{a}{ }^{f} q_{b}{ }^{e} Y^{g} \nabla_{f} \nabla_{e} X_{g}} .
$$

However, since we have

$$
\begin{aligned}
& q_{a}{ }^{f} q_{b}{ }^{e} Y^{g} \nabla_{f} \nabla_{e} X_{g}=q_{a}^{f} Y^{g} \nabla_{f}\left(q_{b}^{e} \nabla_{e} X_{g}\right)-q_{a}^{f} \nabla_{f} q_{b}{ }^{e} Y^{g} \nabla_{e} X_{g} \\
& =q_{a}{ }^{f} Y^{g} \nabla_{f}\left(q_{g}{ }^{h} q_{b}{ }^{e} \nabla_{e} X_{h}+h_{g}{ }^{h} q_{b}{ }^{e} \nabla_{e} X_{h}\right) \\
& \quad-q_{a}^{f} \nabla_{f} q_{b}^{e} Z_{e}+q_{a}{ }^{f} \nabla_{f} q_{b}{ }^{e} X^{g} \nabla_{g} Y_{e} \\
& =q_{a}^{f} Y^{g} \nabla_{f}\left(K_{b g}^{(X)}+\tilde{D}_{b} X_{g}\right)+q_{a}^{f} q_{b}{ }^{e} \nabla_{f} Z_{e} \\
& \quad+q_{a}^{f} \nabla_{f} q_{b}^{e}\left(q_{e}{ }^{h}+h_{e}{ }^{h}\right) X^{g} \nabla_{g} Y_{h} \\
& =-q_{a}^{f} \nabla_{f} Y^{g} K_{b g}^{(X)}+Y^{g} \tilde{D}_{a} \tilde{D}_{b} X_{g}+K_{a b}^{(Z)} \\
& \quad+K_{a c b}\left(X^{g} \nabla_{g} Y^{c}\right)+K_{a b c}\left(X^{g} \nabla_{g} Y^{c}\right) \\
& =-K_{a}^{(Y) g} K_{b g}^{(X)}+Y^{g} \tilde{D}_{a} \tilde{D}_{b} X_{g}+K_{a b}^{(Z)} \\
& \quad-K_{a c b}\left(Y_{e} \tilde{D}^{c} X^{e}\right)+K_{a b c}\left(X^{g} \nabla_{g} Y^{c}\right)
\end{aligned}
$$

and after substituting this result into eq.(238), we finally get the deformation equation (50).

\section{Appendix B}

Remembering that $\epsilon_{a b}$ is defined as

$$
\epsilon_{a b}=n_{a} \ell_{b}-\ell_{a} n_{b} \quad \text { or } \quad \epsilon_{a b}=u_{a} v_{b}-v_{a} u_{b},
$$

it's easy to find

$$
\epsilon_{a}^{c} \epsilon_{c b}=h_{a b}, \quad \epsilon_{a b} \epsilon_{c d}=h_{a d} h_{b c}-h_{a c} h_{b d} .
$$

This also suggests that we can introduce the tensor $\epsilon_{a b}$ without using the local frames. By defining

$$
\bar{X}^{a}=\epsilon^{a b} X_{b}, \quad \bar{Y}^{a}=\epsilon^{a b} Y_{b},
$$

then, we have

$$
X^{a}=\epsilon^{a b} \bar{X}_{b}, \quad Y^{a}=\epsilon^{a b} \bar{Y}_{b}, \quad \bar{X}_{a} \bar{Y}^{a}=-X_{a} Y^{a} .
$$

It's also easy to find $\ell_{a}=\epsilon_{a b} \ell^{b}$ and $n_{a}=-\epsilon_{a b} n^{b}$. This means: $\ell_{a}$ is self-dual, but $n_{a}$ is anti-self-dual. 


\section{Appendix C}

From the definition of the covariant derivative $\tilde{D}$, we have

$$
\begin{aligned}
& \tilde{D}_{a} X_{b}=h_{b}{ }^{d} q_{a}{ }^{c} \nabla_{c} X_{d}=\varepsilon_{I J} e_{b}^{I} e^{J d} q_{a}{ }^{c} \nabla_{c} X_{d} \\
& =\varepsilon_{I J} e_{b}^{I} q_{a}{ }^{c} \nabla_{c}\left(e^{J d} X_{d}\right)-\left(\varepsilon_{I J} e_{b}^{I} q_{a}{ }^{c} \nabla_{c} e^{J d}\right) X_{d} \\
& =D_{a} X^{J} \varepsilon_{I J} e_{b}^{I}+\omega_{a b d} X^{d} \\
& =D_{a} X^{J} \varepsilon_{I J} e_{b}^{I}+\omega_{a} \epsilon_{b d} X^{d},
\end{aligned}
$$

and then

$$
\begin{aligned}
& \tilde{D}_{c} \tilde{D}_{a} X_{b}=q_{c}^{e} q_{a}^{f} h_{b}^{g} \nabla_{e}\left(D_{f} X^{J} \varepsilon_{I J} e_{g}^{I}+\omega_{f} \epsilon_{g d} X^{d}\right), \\
& =\left(D_{c} D_{a} X^{J}\right) \varepsilon_{I J} e_{b}^{I}+D_{a} X^{J} \omega_{c} \epsilon_{b d} \varepsilon_{I J} e^{I d} \\
& +D_{c} \omega_{a} \epsilon_{b d} X^{d}+D_{c} X^{J} \omega_{a} \epsilon_{b d} \varepsilon_{I J} e^{I d}+\omega_{a} \omega_{c} \epsilon_{b d} \epsilon_{e}^{d} X^{e} .
\end{aligned}
$$

So we get eq.(61). Here, we have used the fact that $\tilde{D}_{a} \epsilon_{b c}=0$.

\section{References}

[1] J. D. Bekenstein, Phys. Rev. D 7, 2333 (1973).

[2] S. W. Hawking, Commun. Math. Phys. 43, 199 (1975) [Erratum-ibid. 46, 206 (1976)].

[3] J. M. Bardeen, B. Carter and S. W. Hawking, Commun. Math. Phys. 31, 161 (1973).

[4] R. M. Wald, Living Rev. Rel. 4, 6 (2001) arXiv:gr-qc/9912119.

[5] S.W. Hawking, G.F.R. Ellis, The large scale structure of space time, Cambridge: Cambridge University Press, 1973.

[6] A. Ashtekar and B. Krishnan, Living Rev. Rel. 7, 10 (2004) arXiv:gr-qc/0407042.

[7] T. Jacobson, Phys. Rev. Lett. 75, 1260 (1995) arXiv:gr-qc/9504004.

[8] C. Eling, R. Guedens, and T. Jacobson, Phys. Rev. Lett. 96, 121301 (2006).

[9] S. A. Hayward, Phys. Rev. D 49, 6467 (1994).

[10] S. A. Hayward, Phys. Rev. D 53, 1938 (1996) arXiv:gr-qc/9408002.

[11] S. A. Hayward, Class. Quant. Grav. 15, 3147 (1998) arXiv:gr-qc/9710089.

[12] R. Penrose, Phys. Rev. Lett. 14, 57 (1965). 
[13] A. Ashtekar, C. Beetle and S. Fairhurst, Class. Quant. Grav. 16, L1 (1999) arXiv:gr-qc/9812065.

[14] A. Ashtekar, A. Corichi and K. Krasnov, Adv. Theor. Math. Phys. 3, 419 (2000) arXiv:gr-qc/9905089.

[15] A. Ashtekar, C. Beetle and S. Fairhurst, Class. Quant. Grav. 17, 253 (2000) arXiv:gr-qc/9907068.

[16] A. Ashtekar, S. Fairhurst and B. Krishnan, Phys. Rev. D 62, 104025 (2000) arXiv:gr-qc/0005083.

[17] A. Ashtekar, C. Beetle, O. Dreyer, S. Fairhurst, B. Krishnan, J. Lewandowski and J. Wisniewski, Phys. Rev. Lett. 85, 3564 (2000) arXiv:gr-qc/0006006.

[18] A. Ashtekar and B. Krishnan, Phys. Rev. Lett. 89, 261101 (2002) arXiv:gr-qc/0207080.

[19] A. Ashtekar and B. Krishnan, Phys. Rev. D 68, 104030 (2003) arXiv:gr-qc/0308033.

[20] I. Booth and S. Fairhurst, Phys. Rev. Lett. 92, 011102 (2004) arXiv:gr-qc/0307087.

[21] W. Kavanagh and I. Booth, Phys. Rev. D 74, 044027 (2006) arXiv:gr-qc/0603074.

[22] I. Booth and S. Fairhurst, Phys. Rev. D 75, 084019 (2007) arXiv:gr-qc/0610032.

[23] L. B. Szabados, Class. Quant. Grav. 11, 1833 (1994) arXiv:gr-qc/9402001.

[24] L. Andersson, M. Mars and W. Simon, Phys. Rev. Lett. 95, 111102 (2005) arXiv:gr-qc/0506013.

[25] L. Andersson, M. Mars and W. Simon, Adv. Theor. Math. Phys. 12, 853 (2008) arXiv:0704.2889 [gr-qc].

[26] C. W. Misner and D. H. Sharp, Phys. Rev. 136, B571 (1964).

[27] S.W. Hawking, Gravitational radiation in an expanding universe, J. Math. Phys. 9, 598 (1968).

[28] S. A. Hayward, S. Mukohyama and M. C. Ashworth, Phys. Lett. A 256, 347 (1999) arXiv:gr-qc/9810006.

[29] S. A. Hayward, Phys. Rev. Lett. 93, 251101 (2004) arXiv:gr-qc/0404077.

[30] S. A. Hayward, Phys. Rev. D 70, 104027 (2004) arXiv:gr-qc/0408008. 
[31] H. Bray, S. Hayward, M. Mars and W. Simon, Commun. Math. Phys. 272, 119 (2007) arXiv:gr-qc/0603014.

[32] R. M. Wald, General Relativity, University of Chicago Press, Chicago, 1984.

[33] B. Carter, J. Geom. Phys. 8, 53 (1992).

[34] B. Carter, arXiv:hep-th/9705172.

[35] B. Carter, Int. J. Theor. Phys. 40, 2099 (2001) arXiv:gr-qc/0012036.

[36] E. Gourgoulhon, arXiv:gr-qc/0703035.

[37] T. Damour, Phys. Rev. D 18, 3598 (1978).

[38] E. Gourgoulhon, Phys. Rev. D 72, 104007 (2005) arXiv:gr-qc/0508003.

[39] E. Gourgoulhon and J. L. Jaramillo, Phys. Rev. D 74, 087502 (2006) arXiv:gr-qc/0607050.

[40] E. Gourgoulhon and J. L. Jaramillo, New Astron. Rev. 51, 791 (2008) arXiv:0803.2944 [astro-ph]].

[41] J. D. Brown and J. W. York, Phys. Rev. D 47, 1407 (1993) arXiv:gr-qc/9209012.

[42] K.S.Throne, R.H.Price, and D.A.MacDonald, Black Holes: The Membrane Paradigm, Yale University Press, New Haven, 1986.

[43] M. Parikh and F. Wilczek, Phys. Rev. D 58, 064011 (1998) arXiv:gr-qc/9712077.

[44] C. Eling, I. Fouxon and Y. Oz, Phys. Lett. B 680, 496 (2009) arXiv:0905.3638 [hep-th]].

[45] C. Eling and Y. Oz, JHEP 1002, 069 (2010) [arXiv:0906.4999 [hep-th]].

[46] I. Booth, M. P. Heller and M. Spalinski, Phys. Rev. D 80, 126013 (2009) arXiv:0910.0748 [hep-th]].

[47] R. Mizuno, S. Ohashi and T. Shiromizu, Phys. Rev. D 81, 044030 (2010) arXiv:0911.5560 [gr-qc]].

[48] H. Kodama, Prog. Theor. Phys. 63, 1217 (1980).

[49] A. B. Nielsen and J. H. Yoon, Class. Quant. Grav. 25, 085010 (2008) arXiv:0711.1445 [gr-qc]].

[50] I. Booth and J. Martin, arXiv:1007.1642 [gr-qc].

[51] H. Maeda and M. Nozawa, Phys. Rev. D 77, 064031 (2008) arXiv:0709.1199 [hep-th]]. 
[52] M. Nozawa and H. Maeda, Class. Quant. Grav. 25, 055009 (2008) arXiv:0710.2709 [gr-qc]].

[53] A. L. Besse, Einstein Manifolds, Springer-Verlag, 1987.

[54] S. A. Hayward, Phys. Rev. D 74, 104013 (2006) arXiv:gr-qc/0609008.

[55] S. A. Hayward, Class. Quant. Grav. 24, 923 (2007) arXiv:gr-qc/0611027.

[56] A. V. Frolov and L. Kofman, JCAP 0305, 009 (2003) arXiv:hep-th/0212327.

[57] U. H. Danielsson, Phys. Rev. D 71, 023516 (2005) arXiv:hep-th/0411172.

[58] R. Bousso, Phys. Rev. D 71, 064024 (2005) arXiv:hep-th/0412197.

[59] G. Calcagni, JHEP 0509, 060 (2005) arXiv:hep-th/0507125.

[60] R. G. Cai and S. P. Kim, JHEP 0502, 050 (2005) arXiv:hep-th/0501055.

[61] R. G. Cai, L. M. Cao and N. Ohta, Phys. Rev. D 81, 084012 (2010) arXiv:1002.1136 [hep-th]].

[62] R. G. Cai, L. M. Cao and Y. P. Hu, JHEP0808: 090,2008 [arXiv: 0807.1232[hep-th]]; M. Akbar and R. G. Cai, Phys. Rev. D 75, 084003 (2007) arXiv:hep-th/0609128; R. G. Cai and L. M. Cao, Phys. Rev. D 75, 064008 (2007) arXiv:gr-qc/0611071]; Y. Gong and A. Wang, Phys. Rev. Lett. 99, 211301 (2007) [arXiv:0704.0793 [hep-th]]; S. F. Wu, B. Wang and G. H. Yang, Nucl. Phys. B 799, 330 (2008) arXiv:0711.1209 [hep-th]].

[63] R. G. Cai, Prog. Theor. Phys. Suppl. 172: 100-109, 2008 arXiv:0712.2142[hep-th]]; R. G. Cai and L. M. Cao, Nucl. Phys. B 785, 135 (2007) arXiv:hep-th/0612144; A. Sheykhi, B. Wang and R. G. Cai, Nucl. Phys. B 779, 1 (2007) arXiv:hep-th/0701198; A. Sheykhi, B. Wang and R. G. Cai, Phys. Rev. D 76, 023515 (2007) |arXiv:hep-th/0701261]; X. H. Ge, Phys. Lett. B 651, 49 (2007) arXiv:hep-th/0703253.

[64] T. Padmanabhan, Class. Quant. Grav. 19, $5387 \quad$ (2002) arXiv:gr-qc/0204019; D. Kothawala, S. Sarkar and T. Padmanabhan, Phys. Lett. B 652, 338 (2007) arXiv:gr-qc/0701002; A. Paranjape, S. Sarkar and T. Padmanabhan, Phys. Rev. D 74, 104015 (2006) arXiv:hep-th/0607240; R. G. Cai, L. M. Cao, Y. P. Hu and S. P. Kim, Phys. Rev. D 78, 124012 (2008) arXiv:0810.2610 [hep-th]]; M. Akbar, Chin. Phys. Lett. 24, 1158 (2007) arXiv:hep-th/0702029; M. Akbar and A. A. Siddiqui, Phys. Lett. B 656, 217 (2007); M. Akbar and R. G. Cai, Phys. Lett. B 648, 243 (2007) arXiv:gr-qc/0612089; R. G. Cai and N. Ohta, arXiv:0910.2307 [hep-th].

[65] K. i. Maeda and M. Nozawa, Phys. Rev. D 81, 044017 (2010) arXiv:0912.2811 [hep-th]]; K. i. Maeda and M. Nozawa, Phys. Rev. D 81, 124038 (2010) [arXiv:1003.2849 [gr-qc]]. 
[66] T. Padmanabhan, arXiv:0911.5004 [gr-qc].

[67] T. Padmanabhan, Phys. Rev. D 81, 124040 (2010) arXiv:1003.5665 [grqc]].

[68] E. P. Verlinde, arXiv:1001.0785 [hep-th].

[69] G. Abreu and M. Visser, Phys. Rev. Lett. 105, 041302 (2010) arXiv:1005.1132 [gr-qc]]. 